\title{
PREVISÃO DE VIDA À FADIGA ATRAVÉS DE MODELO BASEADO NA MICROMECÂNICA DE DEFEITOS
}

\author{
TIAGO DE BORTOLI LUCIANO
}

MESTRADO EM CIÊNCIAS MECÂNICAS

DEPARTAMENTO DE ENGENHARIA MECÂNICA 
UNIVERSIDADE DE BRASÍLIA - UnB

FACULDADE DE TECNOLOGIA - FT

DEPARTAMENTO DE ENGENHARIA MECÂNICA - UnB

PREVISÃO DE VIDA À FADIGA ATRAVÉS DE MODELO BASEADO NA MICROMECÂNICA DE DEFEITOS

TIAGO DE BORTOLI LUCIANO

ORIENTADOR: PROF. DR. LUCIVAL MALCHER

DISSERTAÇÃO DE MESTRADO EM CIÊNCIAS MECÂNICAS

PUBLICAÇÂO: ENM.DM-237/2016

BRASÍLIA/DF: SETEMBRO /2016 
UNIVERSIDADE DE BRASÍLIA - UnB

FACULDADE DE TECNOLOGIA - FT

DEPARTAMENTO DE ENGENHARIA MECÂNICA - UnB

PREVISÃO DE VIDA À FADIGA ATRAVÉS DE MODELO BASEADO NA MICROMECÂNICA DE DEFEITOS

TIAGO DE BORTOLI LUCIANO

DISSERTAÇÃO DE MESTRADO SUBMETIDA AO DEPARTAMENTO DE ENGENHARIA MECÂNICA DA FACULDADE DE TECNOLOGIA DA UNIVERSIDADE DE BRASÍLIA, COMO PARTE DOS REQUISITOS NECESSÁRIOS PARA A OBTENÇÃO DO GRAU DE MESTRE EM CIÊNCIA MECÂNICA.

APROVADO POR:

LUCIVAL MALCHER, Doutor, ENM/FT/UnB (Orientador)

HENRIQUE GOMES DE MOURA, Doutor, FGA/UnB (Examinador externo)

JORGE LUIZ DE A. FERREIRA, Doutor, ENM/FT/UnB (Examinador interno)

$\overline{\text { FÁBIO COMES DE CASTRO, Doutor, ENM/FT/UnB (Suplente) }}$

BRASÍLIA/ DF, SETEMBRO/2016 


\section{FICHA CATALOGRÁFICA}

BORTOLI L., TIAGO DE

PREVISÃo DE VIDA À FADIGA ATRAVÉS DE MODELO BASEAdO NA MICROMECÂNICA DE DEFEITOS

027A, 210 x 297 mm (ENM/FT/UnB, Mestre, Ciências Mecânicas, 2016). Dissertação de Mestrado

- Universidade de Brasília. Faculdade de Tecnologia.

Departamento de Engenharia Mecânica.

Programa de Pós-graduação em Ciências Mecânicas.
1. Fadiga Multiaxial
2. Micromecânica de defeitos
3. Plasticidade Cíclica
4. Previsão de vida à fadiga
I. ENM/FT/UnB
II. Título (série)

\section{REFERÊNCIA BILIOGRÁFICA}

DE BORTOLI L., TIAGO (2016). Previsão de Vida à Fadiga Através de Modelo Baseado na

Micromecânica de Defeitos. Dissertação de Mestrado, Publicação ENM.DM-237a/16,

Departamento de Engenharia Mecânica, Universidade de Brasília, Brasília, DF,118 p.

\section{CESSÃO DE DIREITOS}

AUTOR: Tiago de Bortoli Luciano

TÍTULO: Previsão de vida à fadiga através de modelo baseado na micromecânica de defeitos

GRAU: Mestre em Ciências Mecânicas $\quad$ ANO: 2016

É concedida à Universidade de Brasília permissão para reproduzir cópias desta dissertação de mestrado e para emprestar ou vender tais cópias somente para propósitos acadêmicos e científicos. O autor reserva outros direitos de publicação e nenhuma parte dessa dissertação de mestrado pode ser reproduzida sem autorização por escrito do autor.

Tiago de Bortoli Luciano

Universidade De Brasília - Faculdade de Tecnologia - Departamento de Engenharia Mecânica Brasília - DF - Brasil

CEP.: 70910-900 


\section{AGRADECIMENTO}

Agradeço primeiramente a Deus e aos meus anjos da guarda, por sempre me guiado com muita luz, sabedoria e saúde ao pleno cumprimento dos objetivos intrínsecos ao desenvolvimento humano.

A minha família que sempre apoiou e incentivou minha busca pelo estudo e evolução do conhecimento, em especial, aos meus pais que solidificaram minha educação com base em valores éticos e minha amada esposa que compartilhou cada passo desta etapa, cada gota de suor, cada ausência, cada noite em que não pude estar ao seu lado e cada segundo sem minha atenção aproveitado, sempre compreendendo o bem maior e me incentivando a seguir e concluir cada desafio que a vida nos impor.

Ao professor Dr. Lucival Malcher, meu orientador e amigo, quem me ajudou a abrir a porta do desafio que neste trabalho se finda, sempre iluminando meus passos e compartilhando conhecimentos fundamentais para uma formação que me permitisse cumprir esta meta e seguir em frente para a continuidade de estudos que venham a somar resultados duradouros ao meio acadêmico e industrial.

Ao Profo Dr. Edgar Mamiya, Profa Dra. Aline, Profo Dr. Jorge e demais professores que contribuíram para minha formação acadêmica ao longo destes dois anos, agradeço-Ihes por cada momento de troca de experiência, por cada ensinamento compartilhado e por todo o incentivo ao aprendizado.

Aos meus amigos, obrigado por cada discussão, por cada madrugada, por toda paciência e, principalmente, por fazerem parte desta minha jornada.

Tiago de Bortoli Luciano 
"Aprenda a aprender" COLL C., 1994. 


\section{RESUMO}

A análise de vida para componentes de equipamentos e estruturas sob condições adversas de solicitação mecânica é um passo importante e que merece muita atenção no projeto de componentes metálicos. Neste contexto, a previsão da vida útil pode ser feita através da utilização da Mecânica dos Meios Contínuos, ou seja, a motivação do presente trabalho nasce a partir do interesse em utilizar modelos constitutivos embasados na micromecânica de defeitos e integrados a modelos de plasticidade cíclica, com intuito de prever a vida à fadiga em materiais dúcteis. A partir de dados experimentais, serão apresentados estudos comparativos dos resultados para a previsão da falha obtidos por modelos embasados na micromecânica de defeitos, a saber: Modelo de Gurson acoplado a lei de endurecimento cinemático de Armstrong e Frederick. Para tanto, o citado modelo teve suas parcelas matemática e numérica desenvolvidos em uma plataforma acadêmica e linguagem FORTRAN, onde com a última se elaborou os algoritmos de retorno e de Newton-Raphson capazes de atualizar as variáveis internas do problema. Por fim, o modelo aqui implementando permitiu confrontar resultados experimentais de outras literaturas e inferir sobre a precisão da utilização da micromecânica do defeito para o emprego em modelos de previsão de vida à fadiga.

Palavras chaves: micromecânica de defeitos; previsão de vida à fadiga; porosidade para o dano; modelo de Gurson; plasticidade cíclica. 


\section{ABSTRACT}

Life analysis for equipment components and structures under severe mechanical stress is an important step that deserves much attention in the metal components design. In this context, the prediction of service life may be made by using the Continuum Mechanics, ie the present study is motivated rises from the interest in use constitutive models grounded in micromechanics defects and integrated with cyclic plasticity models, in order to predict the fatigue life in ductile materials. From experimental data, will be presented comparative studies of the results for the prediction of failure obtained by models grounded in micromechanics defects, namely: Gurson model coupled to kinematic hardening law of Armstrong and Frederick. Therefore, the model mentioned had their mathematical and numerical plots developed in an academic platform and FORTRAN language, where with the last one was produced the algorithm of return and the NewtonRaphson able to update the internal variables of the problem. Finally, the model here implementing allowed confront experimental results of other literature and infer about the accuracy of the use of micromechanical default for employment in fatigue life prediction models.

Key words: micro mechanical damage; life fatigue prediction; porosity for the damage; Gurson model; cyclic plasticity. 


\section{SUMÁRIO}

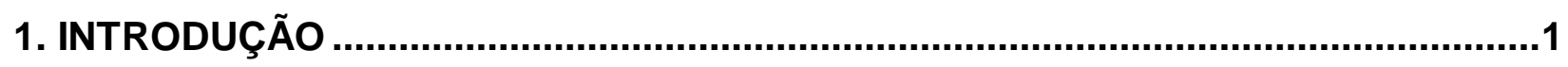

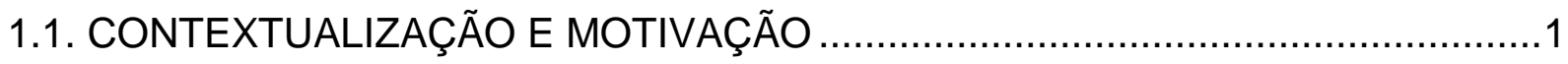

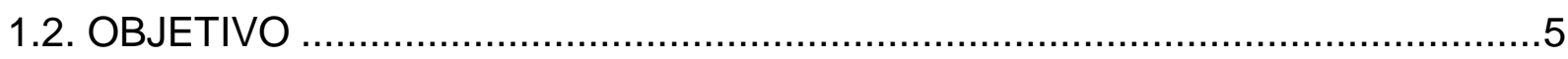

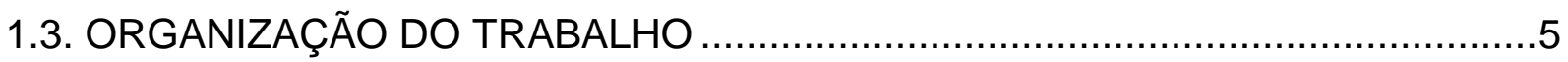

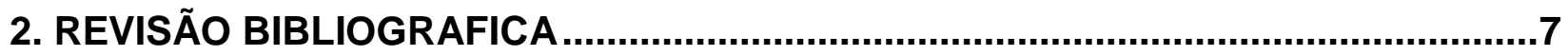

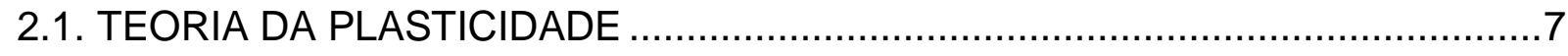

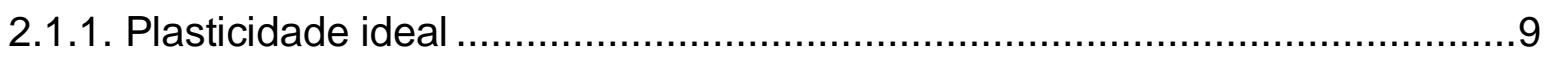

2.1.2. Endurecimento isotrópico .................................................................10

2.1.3. Endurecimento cinemático .................................................................11

2.2. MODELO PARA PLASTICIDADE CÍCLICA …............................................12

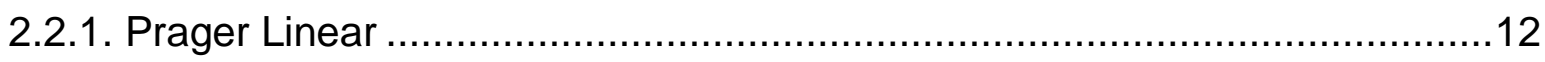

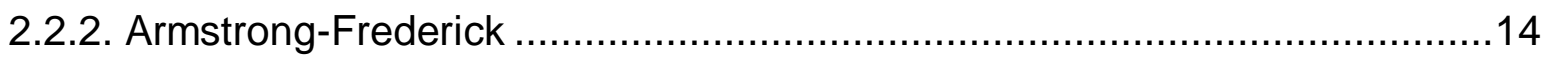

2.2.3. Chaboche

2.3. MODELOS BASEADOS NA MICROMECÂNICA DE DEFEITOS .......................18

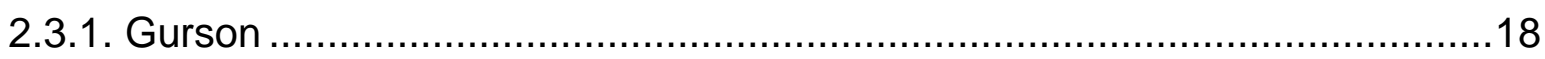

2.3.2. Gurson-Tvergaard-Needleman (modelo GTN).......................................23

3. DESENVOLVIMENTO DO MODELO ELASTO-PLÁSTICO DE GURSON COM

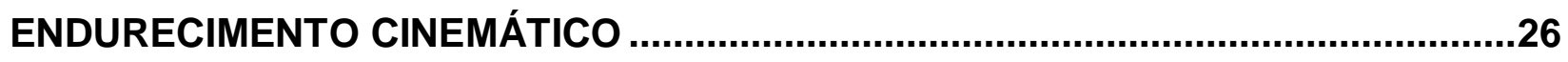

3.1. DESENVOLVIMENTO DO MODELO MATEMÁTICO ......................................26

3.2. DESENVOLVIMENTO DO MODELO NUMÉRICO ........................................31

3.2.1. Algoritmo de retorno: Gurson com Armstrong-Frederick............................32

3.2.2. Algoritmo de Newton-Raphson: Sistema de equações não linear ................36 
4. RESULTADOS E DISCUSSÕES

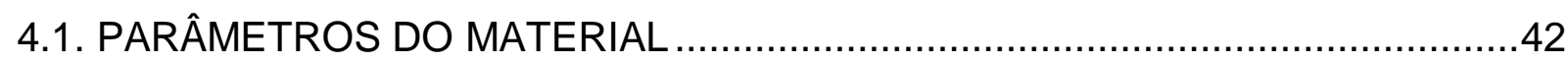

4.2. TRAJETÓRIAS PROPORCIONAIS E NÃO PROPORCIONAIS .........................45

4.2.1. Previsão de vida a fadiga para trajetórias uniaxiais ..................................46

4.2.2. Previsão de vida a fadiga para trajetórias multiaxiais proporcionais ............52

4.2.3. Previsão de vida a fadiga para trajetórias multiaxiais não proporcionais .....58

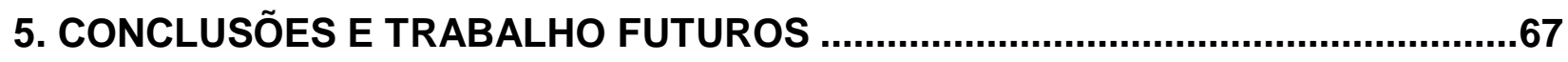

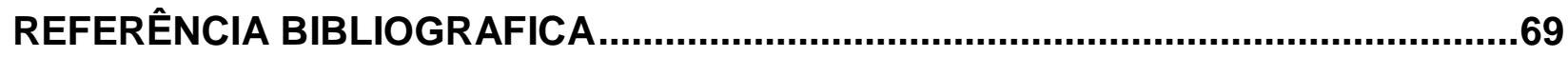

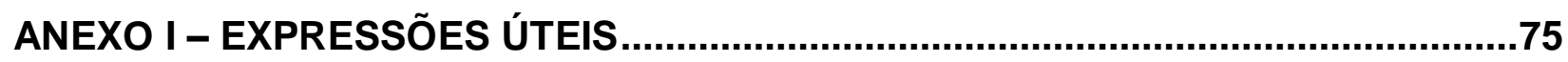

ANEXO II - ALGORITMO DE INTEGRAÇÃO NUMÉRICA......................................77 


\section{LISTA DE FIGURAS}

Figura 1. Representação do efeito de Bauschinger. Adaptado de Jiang, Y. \& Zhang, J., 2008.

Figura 2. Ensaio numérico de previsão de vida a fadiga. Fonte: Lestriez, P., et al, 2007...4

Figura 3. Curva Tensão/Deformação genérica para um material dúctil. Fonte: Adaptado de

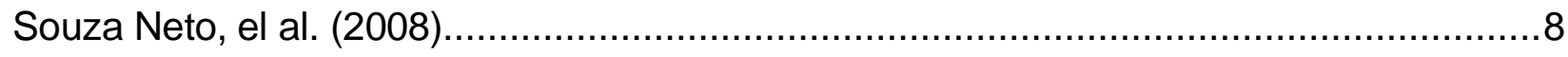

Figura 4. Plasticidade ideal. Fonte: Adaptado Souza Neto, el al, 2008..........................9

Figura 5. Endurecimento isotrópico não linear. Fonte: Adaptado Souza Neto, el al, 2008.

Figura 6. Endurecimento cinemático não linear. Fonte: Adaptado Souza Neto, el al, 2008.

Figura 7. Exemplo de curva tensão-deformação para o modelo de Prager. Diferença entre o laço de histerese experimental e numérico. Fonte: Adaptado de Paul, S.K., et al, 2010.

Figura 8. Exemplo de curva tensão-deformação para o modelo de Armstrong e Frederick. Diferença entre o laço de histerese experimental e numérico. Fonte: Adaptado de Paul, S.K., et al, 2010.

Figura 9. Exemplo de curva tensão-deformação para o modelo de Chaboche. Diferença entre o laço de histerese experimental e numérico. Fonte: Adaptado de Paul, S.K., et al, 2010.

Figura 10. Representação esquemática do elemento representativo com um vazio esfério. Adaptado de Gurson, 1977.

Figura 11. Representação esquemática do processo de nucleação, crescimento e coalescência dos micros vazios e a relação com o carregamento macroscópico. Fonte: Pineau e Pardoen, 2003. 19

Figura 12. Imagem ótica da nucleação de um vazio em escala manométrica. Zheng, H., et al. (2014). 
Figura 13. Gráfico comparativo da evolução da porosidade efetiva em função da porosidade inicial ( $f o=0$ ou $f o>0$ ), e da deformação plástica equivalente. .23

Figura 14. Gráfico comparativo da evolução da porosidade efetiva nos modelos de Gurson e GTN.

Figura 15. Trajetória uniaxial de tração-compressão imposta para calibração. Fonte: Jiang et al, 2007.

Figura 16. Evolução da porosidade em função do ciclo de vida do Aço 304

Figura 17. Evolução da porosidade em função do ciclo de vida do Aço S460N. .48

Figura 18. Evolução da porosidade em função do ciclo de vida do Alumínio 6061-T6.....49

Figura 19. Comparação entre a vida observada experimentalmente e a vida estimada numericamente pelo modelo de Gurson. Aço 304 em trajetória uniaxial..... .51

Figura 20. Comparação entre a vida observada experimentalmente e a vida estimada numericamente pelo modelo de Gurson. Aço S460N em trajetória uniaxial. 51

Figura 21. Comparação entre a vida observada experimentalmente e a vida estimada numericamente pelo modelo de Gurson. Alumínio 6061-T6 em trajetória uniaxial. .52

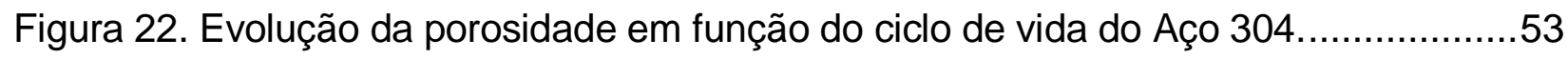

Figura 23. Evolução da porosidade em função do ciclo de vida do Aço S460N...............54

Figura 24. Evolução da porosidade em função do ciclo de vida do Alumínio 6061-T6.....55 Figura 25. Comparação entre a vida observada experimentalmente e a vida estimada numericamente pelo modelo de Gurson. Aço 304 em trajetória multiaxial proporcional...56

Figura 26. Comparação entre a vida observada experimentalmente e a vida estimada numericamente pelo modelo de Gurson. Aço S460N em trajetória multiaxial proporcional.

Figura 27. Comparação entre a vida observada experimentalmente e a vida estimada numericamente pelo modelo de Gurson. Alumínio 6061-T6 em trajetória multiaxial

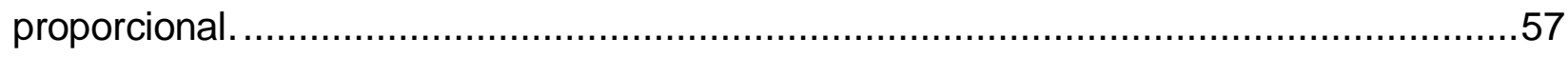

Figura 28. Evolução da porosidade em função do ciclo de vida do Aço $304 \ldots \ldots \ldots \ldots . . . . . . . . . .58$ 
Figura 29. Evolução da porosidade em função do ciclo de vida do Aço S460N.

Figura 30. Evolução da porosidade em função do ciclo de vida do Alumínio 6061-T6.....60

Figura 31. Comparação entre a vida observada experimentalmente e a vida estimada numericamente pelo modelo de Gurson. Aço 304 em trajetória multiaxial não proporcional.

Figura 32. Comparação entre a vida observada experimentalmente e a vida estimada numericamente pelo modelo de Gurson. Aço $S 460 \mathrm{~N}$ em trajetória multiaxial não proporcional.

Figura 33. Comparação entre a vida observada experimentalmente e a vida estimada numericamente pelo modelo de Gurson. Alumínio 6061-T6 em trajetória multiaxial não proporcional.

Figura 34. Evolução da porosidade no primeiro ciclo. Aço 304 em trajetória multiaxial Caso 3 / Tab. 7.

Figura 35. Evolução da porosidade no primeiro ciclo. Aço 304 em trajetória multiaxial Caso 3 / Tab. 7. .63

Figura 36. Evolução da porosidade no primeiro ciclo. Aço $\$ 460 \mathrm{~N}$ em trajetória multiaxial Caso 4 / Tab. 6. 64

Figura 37. Evolução da porosidade no primeiro ciclo. Aço S460N em trajetória multiaxial Caso 4 / Tab. 6.

Figura 38. Evolução da porosidade no primeiro ciclo. Alumínio 6061 em trajetória multiaxial - Caso 3 / Tab. 7.

Figura 39. Mapeamento da evolução de incrementos dentro da trajetória multiaxial não proporcional aplicada. .65

Figura 40. Visão geral da comparação entre a vida observada experimentalmente e a vida estimada numericamente pelo modelo de Gurson para todos os materiais e trajetórias propostas. 


\section{LISTA DE TABELAS}

Tabela 1. Resultados da calibração por amplitude para o modelo de Armstrong-Frederick e parâmetros da curva de Ramberg-Osgood. Fonte: adaptado, Pereira, 2014. .43

Tabela 2. Resultados de identificação de vida experimental para o aço 304. Fonte: Itoh (2001). 44

Tabela 3. Resultados de identificação de vida experimental para o aço S460N. Fonte: Jiang, et al. (2007).

Tabela 4. Resultados de identificação de vida experimental para o alumínio 6061-T6. Fonte: Itoh (2001).

Tabela 5. Resumo das propriedades dos materiais envolvidos na análise. 45

Tabela 6. Históricos de carregamento impostos para o aço S460N. Fonte: Jiang et al., 2007.

Tabela 7. Históricos de carregamento impostos para o aço 304 e a liga de alumínio 6061 T6. Fonte: Itoh, 2001.

Tabela 8. Análise comparativa da vida numérica para vida experimental em diferentes trajetórias uniaxiais. Aço 304 (Itoh, 2001).

Tabela 9. Análise comparativa da vida numérica para vida experimental em diferentes trajetórias uniaxiais. Aço S460N (Jiang, et al. 2007).

Tabela 10. Análise comparativa da vida numérica para vida experimental em diferentes trajetórias uniaxiais. Alumínio 6061-T6 (Itoh, 2001). .49

Tabela 11. Análise comparativa da vida numérica para vida experimental em diferentes trajetórias multiaxiais proporcionais. Aço 304 (Itoh, 2001). .53

Tabela 12. Análise comparativa da vida numérica para vida experimental em diferentes trajetórias multiaxiais proporcionais. Aço S460N (Jiang, et al. 2007).

Tabela 13. Análise comparativa da vida numérica para vida experimental em trajetória multiaxial proporcional. Alumínio 6061-T6 (Itoh, 2001). 
Tabela 14. Análise comparativa da vida numérica para vida experimental em diferentes trajetórias multiaxiais não proporcionais. Aço 304 (Itoh, 2001)..................................58

Tabela 15. Análise comparativa da vida numérica para vida experimental em diferentes trajetórias multiaxiais não proporcionais. Aço S460N (Jiang, et al. 2007).....................59

Tabela 16. Análise comparativa da vida numérica para vida experimental em trajetória multiaxial não proporcional. Alumínio 6061-T6 (Itoh, 2001)......................................60 


\section{LISTA DE QUADROS}

Quadro 1. Resumo do modelo matemático de Gurson com endurecimento cinemático de

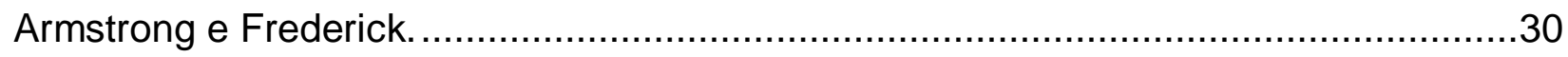

Quadro 2. Resumo do modelo numérico de Gurson com endurecimento cinemático de

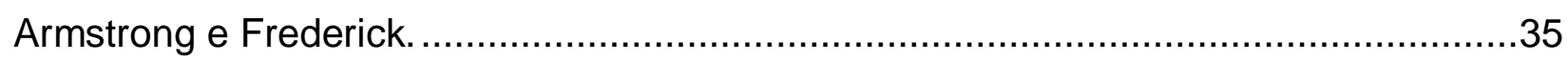

Quadro 3. Algoritmo de resolução do sistema não linear para o modelo de Gurson com endurecimento cinemático de Armstrong e Frederick. .................................................41 


\section{LISTA DE SÍMBOLOS}

$\sigma_{y}=$ Tensão de escoamento;

$p=$ Pressão hidrostática (primeiro invariante do tensor tensão);

$\boldsymbol{\beta}=$ Tensor das tensões cinemáticas;

$q_{n}=$ Parâmetros do material;

$\phi=$ Função de escoamento;

$\dot{\varepsilon}^{p}=$ Lei de fluxo plástico;

$\dot{\gamma}=$ Multiplicador plástico;

$\boldsymbol{N}=$ Vetor de fluxo plástico;

$H=$ Módulo de endurecimento isotrópico;

$H^{k}=$ Módulo de endurecimento cinemático;

$\dot{\bar{\varepsilon}}^{p}=$ Taxa de deformação plástica;

$b=$ Constante material;

$\boldsymbol{\varepsilon}=$ Tensor das deformações;

$\boldsymbol{\varepsilon}^{\boldsymbol{e}}=$ Tensor das deformações elásticas;

$\boldsymbol{\varepsilon}^{\boldsymbol{p}}=$ Tensor das deformações plásticas;

$\boldsymbol{\sigma}=$ Tensor tensão de Cauchy;

$\mathbb{D}^{e}=$ Tensor elástico isotrópico;

$\boldsymbol{S}=$ Tensor das tensões desviadoras;

$\boldsymbol{\eta}=$ Tensor relativo;

$J_{2}(\boldsymbol{\eta})=$ Segundo invariante do tensor relativo;

$\boldsymbol{\varepsilon}_{n+1}^{\boldsymbol{e}}=$ Tensor das deformações elásticas no pseudo - tempo $t_{n+1}$; 
$\boldsymbol{\varepsilon}_{n+1}^{\boldsymbol{p}}=$ Tensor das deformações plásticas no pseudo - tempo $t_{n+1}$;

$\boldsymbol{\sigma}_{n+1}=$ Tensor tensão de Cauchy no pseudo - tempo $t_{n+1}$;

$p_{n+1}=$ Pressão hidrostática no pseudo - tempo $t_{n+1} ;$;

$\boldsymbol{\varepsilon}_{n+1}^{\boldsymbol{e}^{\boldsymbol{T}}}=$ Tensor das deformações elásticas tentativa;

$\boldsymbol{\varepsilon}_{n+1}^{\boldsymbol{p}^{\boldsymbol{T}}}=$ Tensor das deformações plásticas tentativa;

$\boldsymbol{\sigma}_{n+1}^{\boldsymbol{T}}=$ Tensor tensão tentativa;

$\boldsymbol{S}_{n+1}^{\boldsymbol{T}}=$ Tensor das tensões desviadoras tentativa;

$\boldsymbol{\beta}_{n+1}=$ Tensor das tensões cinemáticas tentativa;

$\boldsymbol{\varepsilon}_{d}^{\boldsymbol{p}}=$ Tensor plástico desviador;

$\varepsilon_{v}^{p}=$ Tensor plástico volumétrico;

$f=$ Fração volumétrica de vazios;

$f_{c}=$ Volume crítico do vazio;

$V_{\text {VAZIO }}=$ Volume do vazio;

$V_{E R}=$ Volumero do elemento representativo;

$V_{m}=$ Volumero da matriz de material;

$\rho=$ Densidade do elemento representativo;

$G=$ Módulo de cisalhamento do material;

$k=$ Módulo volumétrico do material;

$\sigma_{1}, \sigma_{2}$ e $\sigma_{3}=$ Espaço das tensões principais;

$\boldsymbol{I}=$ Tensor identidade de segunda ordem;

$R_{(*)}=$ Equacao residual; 


\section{INTRODUÇÃO}

\subsection{CONTEXTUALIZAÇÃO E MOTIVAÇÃO}

A partir das últimas décadas, o avanço tecnológico e o uso de técnicas computacionais baseadas não apenas no Método dos Elementos Finitos, mas também em Métodos de Elementos de Contorno e das Diferenças Finitas passaram a fundamentar abordagens para a solução numérica de problemas da mecânica dos sólidos, tanto na academia, quanto na indústria. (Souza Neto, et al, 2008)

Estes tipos de aplicações estavam inicialmente direcionados para a modelagem do comportamento de sólidos em áreas convencionais da engenharia, onde era possível apenas a análise numérica de componentes através de softwares complexos e que normalmente estavam associados a utilizadores altamente especializados.

Contudo, a partir da crescente demanda industrial por modelos precisos de sólidos, alimentada pelo aumento constante da intenção em reduzir os custos de processamento e, ao mesmo tempo, a evolução das técnicas de plasticidade computacional, tornou-se possível o desenvolvimento de pacotes de software refinados e com um considerável grau de automação que são, hoje, rotineiramente empregados por um grande número de engenheiros e cientistas. (Souza Neto, et al, 2008)

Atualmente, a gama de aplicações para os problemas práticos de interesse da indústria e o número de técnicas que são aplicadas com níveis aceitáveis de capacidade preditiva é muito ampla, indo desde aplicações de engenharia tradicionais até ações como o mapeamento de possíveis falhas de estrutura de um projeto de engenharia, sendo possível a obtenção de resultados, por exemplo, dos pontos que tendem a concentrar tensões e fraturas que ocasionariam falhas a determinados componentes.

A partir da obtenção de tais resultados o engenheiro envolvido com trabalhos que demandam o uso das teorias constitutivas da plasticidade deverá selecionar qual modelo constitutivo melhor define as combinações de tensão para o carregamento previsto no 
projeto, sendo que essa escolha, associada a uma função de escoamento coerente ao mapeamento do material aplicado no componente discretizado, é essencial para uma previsão que convirja em resultados plausíveis com a realidade. (Gurson, 1977).

Dentre os modelos constitutivos clássicos, citam-se os associados com os critérios de escoamento estabelecidos por: Tresca (1868); von Mises (1913); MohrCoulomb (1900); e Drucker-Prager (1952) (Souza Neto, et al, 2008). Sendo que a partir desses modelos a evolução das teorias constitutivas se deu em função das variações dos componentes básicos citados, por exemplo, em casos onde há a presença de carregamentos cíclicos, um modelo constitutivo elastoplástico que não contempla o incremento do chamado efeito de Bauschinger em sua formulação, terá certamente sua capacidade preditiva prejudicada e, provavelmente, apresentará em sua saída um mapeamento do comportamento cinemático do material que não representa a realidade física deste.

Jiang, Y. e Zhang, J. (2008) define o efeito Bauschinger como um fenômeno pelo qual durante a deformação plástica de um material ocorre o aumento de sua tensão de escoamento na direção do fluxo de plástico e a diminui no outro sentido. A Figura 1 esquematiza este efeito, onde após um carregamento cíclico, o limite de escoamento do material passa do ponto A para o ponto B.

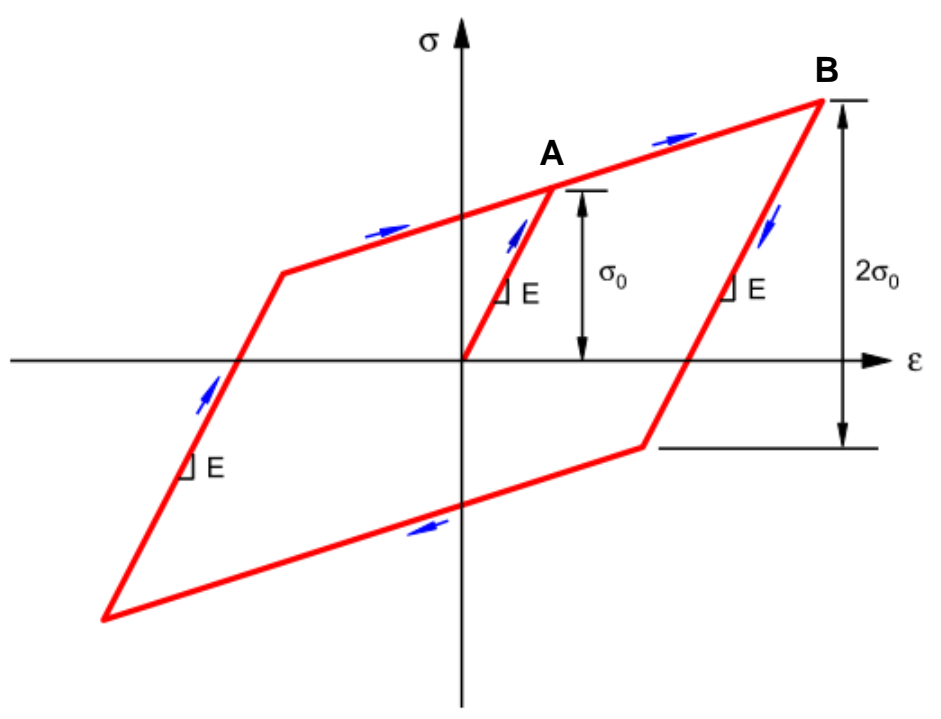

Figura 1. Representação do efeito de Bauschinger. Adaptado de Jiang, Y. \& Zhang, J., 2008. 
Para a análise de casos submetidos a carregamentos cíclicos em regime plástico, destacam-se os modelos de plasticidade cíclica de: Prager (1956); Armstrong-Frederick (1966); Chaboche (1986); Ohno-Wang (1993); e Jiang (1996).

Dessa forma, componentes que tenham sua aplicação em situações de carregamentos cíclicos estão sujeitos a sofrerem falha através da fadiga do material que os compõem, uma vez que o movimento repetitivo de trabalho ocasiona o aparecimento de uma variável conhecida por dano interno. Voyiadjis, George Z., et al. (2012), define dano interno como a presença e a evolução de defeitos - trincas e/ou vazios ao nível microscópico - que pode, eventualmente, levar a fratura de um determinado material.

Destaca-se que em muitas aplicações de engenharia, particularmente naquelas onde as partes mecânicas são submetidas a condições severas, a vida útil dos componentes é um item de informação crucial e que deve ser cuidadosamente considerado durante o processo de design e projeto. Em tais casos, a capacidade do engenheiro em prever a falha mecânica torna-se um fator ligado diretamente ao sucesso do projeto e, em algumas aplicações, é a partir destas previsões que todo um plano de manutenção industrial é traçado, sendo definidas paradas programadas para manutenção e, consequentemente, chegando a diminuição de ocorrências relacionadas a imprevistos que normalmente incorrem em danos econômicos.

Assim, considerando o rápido desenvolvimento das técnicas de mecânica computacional e seguindo com o crescente conhecimento dos mecanismos de análise de danos internos, a possibilidade de previsão de falhas em uma ampla gama de materiais tornou possível a capacidade de predição da evolução da deterioração interna de um determinado componente analisado. Até então, a maior parte dos estudos direcionados para predição de vida a fadiga eram baseados em metodologias empíricas e fundamentadas em experiências acumuladas durante longos períodos de tempo, onde a previsão de falha era conseguida através da realização de testes sistemáticos de modelos reais que tinham suas condições de serviço reproduzidas em laboratório, normalmente este tipo de ensaio demanda um alto custo operacional e financeiro. 
Esse campo de aplicação da mecânica dos sólidos é conhecido como Mecânica do Dano Contínuo - Continuum Damage Mechanics - (CDM) e se mostra uma alternativa para realização da previsão realista da vida de um determinado componente.

Um exemplo de aplicabilidade da plasticidade cíclica se dá em modelos com a capacidade de captura da evolução do dano e do efeito das tensões ligadas ao endurecimento cinemático, sendo possível, a partir destas variáveis, a simulação de um estudo de vida à fadiga do corpo, conforme ilustrado na Figura 2, proveniente de um trabalho de Lestriez, P., et al. (2007).

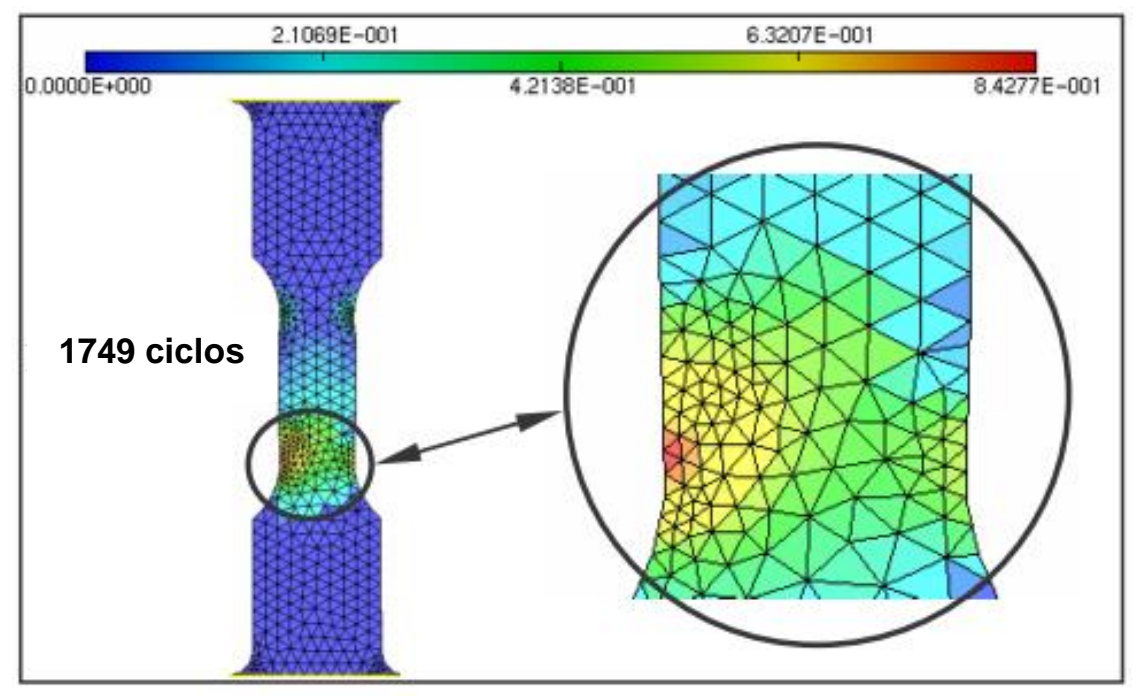

Figura 2. Ensaio numérico de previsão de vida a fadiga. Fonte: Lestriez, P., et al, 2007.

Em conjunto as propostas para modelos da mecânica do dano contínuo (Lemaitre, 1985:1990), outras novas teorias de constitutivas puderam ser propostas, sendo estas com base na micromecânica de defeitos, onde se pode elencar como os principais modelos para esta abordagem: o de Gurson (1977), que conta com uma versão modificada por Gurson-Tvergaard-Needleman - GTN - (Brunig, M., \& Gerke, S., 2011).

Assim, valendo-se da contextualização até aqui exposta, observa-se que a análise da integridade de estruturas através da Mecânica do Dano Contínuo e da plasticidade computacional vem sendo objeto de estudo há várias décadas, no entanto, nos últimos anos esta área de pesquisa tomou um impulso considerável, gerando uma grande quantidade de trabalhos científicos que efetivamente estão sendo aplicados em problemas industriais (Chaboche,1980; Plumtree, 1989). 
Nessa linha, com intuito de análisar materiais dúcteis por meio de uma abordagem ainda pouco empregada, a motivação do presente trabalho nasce a partir do interesse em utilizar um modelo constitutivo embasado na micromecânica de defeitos e integrado a um modelo de plasticidade cíclica, para previsão de vida a fadiga de corpos expostos a carregamentos uniaxiais, multiaxiais proporcionais e multiaxiais não proporcionais.

\subsection{OBJETIVO}

Este trabalho tem como objetivo analisar, por meio de um modelo de micromecânica de defeitos, em carregamento cíclico e com endurecimento cinemático acoplado, o grau de convergência e confiabilidade da previsão de vida à fadiga numérica em comparação com uma determinada experimentalmente.

Para o pleno cumprimento do objetivo proposto, o presente trabalho atenderá em seu desenvolvimento a realização de metas como a elaboração o modelo matemático formulado por Gurson (1977) em conjunto com a lei endurecimento cinemático de Armstrong \& Frederick (1966), além da criação de um esquema implícito de integração numérica capaz de atualizar as variáveis internas conhecidas do problema.

Em seguida, o modelo numérico encontrado será implementado em rotina FORTRAN através do compilador utilizado para simulações em um ambiente de elementos finitos acadêmico.

Por fim, a partir do modelo sugerido, serão inseridos resultados experimentais que permitirão que as saídas numéricas de previsão de vida a fadiga sejam aferidas e analisadas quando a sua convergência e precisão.

\subsection{ORGANIZAÇÃO DO TRABALHO}

Visando atender aos objetivos gerais e específicos expostos no tópico anterior, o presente trabalho prevê que sua organização iniciará, no Capítulo 1, com uma 
contextualização geral do tema proposto, além da explanação de todos os elementos que vieram a motivar a elaboração do estudo aqui desenvolvido. O Capítulo 1 conta ainda com a enumeração das metas que serão atingidos no decorrer do texto.

Passada a contextualização motivacional, o Capítulo 2 traz os conceitos mínimos necessários para uma boa interpretação do tema em questão, abordando tópicos deste a Teoria da Plasticidade até a definição dos modelos mais clássicos de plasticidade cíclica e modelos baseados na micromecânica de defeitos como, por exemplo, Gurson e GTN.

A partir do Capítulo 3 todas as definições e delimitações do trabalho já estarão tomadas, iniciando-se a formulação matemática e numérica do modelo escolhido para o trabalho. Esse capítulo apresentará também a definição do estado tentativa e o algoritmo de integração implícita que será responsável pela atualização das variáveis internas do problema. Durante esse capítulo e quando da conclusão da apresentação de cada modelo, este será resumido dentro de uma caixa que consolidará suas informações básicas.

Com a aplicação dos modelos desenvolvimentos e já detendo o conhecimento técnico científico suficiente para análise, o Capítulo 4 vem para expor toda abordagem já realizada em resultados de curvas numéricas de previsão de vida a fadiga em comparação com outras capturadas experimentalmente. A citada apresentação dos resultados permitirá, ainda no Capítulo 4, a discussão dos valores encontrados e identificação da qualidade do modelo proposto.

O Capítulo 5 consolidará toda a discussão do presente trabalho em função de uma observação crítica entre os objetivos gerais e específicos inicialmente propostos para com os resultados de fato alcançados. Com base na referida análise serão expostas as considerações finais sobre o tema e levantadas sugestões de trabalhos futuros que poderão contribuir com a sequência e evolução do tema aqui abordado.

Por fim, após o Capítulo 5 haverá a apresentação, na forma de anexos, de todos as rotinas programadas e detalhamento matemático das derivadas que deram origem ao sistema de atualização de equações apresentado no Capítulo 3. 


\section{REVISÃO BIBLIOGRAFICA}

\subsection{TEORIA DA PLASTICIDADE}

Souza Neto, el al. (2008), define que a teoria da plasticidade fornece uma visão geral para a descrição da evolução constitutiva do comportamento mecânico de uma importante classe de materiais. Basicamente, a teoria da plasticidade se concentra em sólidos que, após terem sido submetidos a um carregamento, sofrem deformações plásticas, ou seja, mantêm deformações permanentes mesmo após a carga completamente cessada.

Para o entendimento da teoria da plasticidade de forma prática observa-se, na Figura 3, a representação de um gráfico tensão $x$ deformação proveniente do ensaio mecânico de um corpo, o qual inicialmente sofre a aplicação de uma tensão $\sigma_{0}$ suficiente para que seu limite de escoamento inicial $\sigma_{y_{0}}$ seja ultrapassado, ocasionando assim a entrada do material em análise no campo das deformações plásticas, ou seja, depois de cessada a carga aplicada sobre o corpo, este não mais retornará para sua posição zero, mas sim, sofrerá um alongamento permanente $\varepsilon_{1}$.

Esse fato ocasiona um endurecimento do material e consequentemente o aumento do módulo do limite de escoamento do material $\sigma_{y_{1}}$, de forma que para um novo ciclo de carregamento, desta vez em maior intensidade $\sigma_{1}$, nota-se que a curva de característica do corpo passa a seguir por uma nova trajetória, $A$.

Destaca-se ainda que a partir de um corpo de prova já danificado por algum carregamento anterior que tenha ultrapassado seu limite elástico, como no presente exemplo - Figura 3 -, o processo de endurecimento citado ocasiona a alteração dos parâmetros elastoplásticos originais do material, tendo seu envelope de falhas aumentado. (Souza Neto, et al, 2008) 


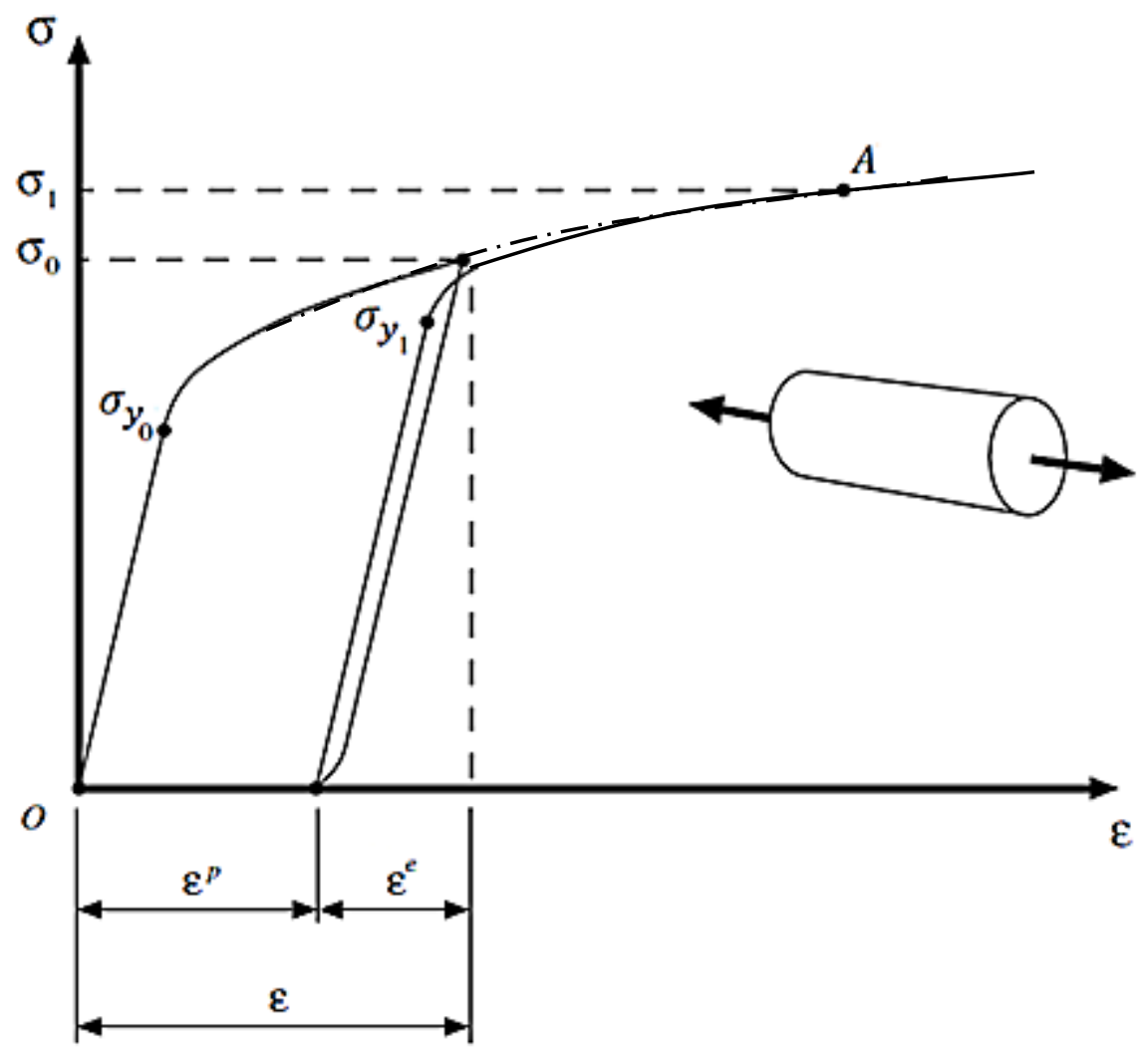

Figura 3. Curva Tensão/Deformação genérica para um material dúctil. Fonte: Adaptado de Souza Neto, el al. (2008).

A partir da Figura 3 nota-se que o comportamento dessa curva segue o conceito citado por Souza Neto, el al. (2008), o qual define que o endurecimento é caracterizado pela dependência do limite de escoamento do material em relação ao histórico de carregamento e deformação plástica a que foi submetido sendo que, em modo geral, o endurecimento pode ser modelado em regimes de: plasticidade ideal, endurecimento isotrópico; ou endurecimento cinemático. Esses conceitos que serão explorados nos tópicos seguintes.

Por fim, se estabelece que os componentes básicos de um modelo constitutivo elastoplástico são sua lei elástica, expressa pela lei de Hooke generalizada, equação 2.1.1 e a observação da decomposição aditiva das deformações, equação 2.1.2. Sendo estes complementados por: um critério de escoamento; uma regra de fluxo de plástico, que define a evolução da deformação plástica; e uma lei de endurecimento, que caracteriza a evolução do limite de escoamento. 


$$
\sigma=\mathbb{D}^{e}: \varepsilon^{e}
$$

onde $\sigma$ expressa o tensor tensão de Cauchy, $\mathbb{D}^{e}$ o tensor constitutivo da matriz elástica do material e $\varepsilon^{e}$ a parcela elástica do tensor das deformações.

$$
\varepsilon=\varepsilon^{e}+\varepsilon^{p}
$$

onde $\varepsilon$ expressa do tensor das deformações sendo, dentro de sua propriedade aditiva, suas parcelas elásticas e plásticas expressas respectivamente por $\varepsilon^{e}$ e $\varepsilon^{p}$.

\subsubsection{Plasticidade ideal}

O endurecimento é dito "perfeitamente plástico" quando não é permitido o encruamento do material, ou seja, o nível de tensão de escoamento não tem nenhuma dependência com o grau de plasticidade aplicado. Nesses casos, o campo de escoamento permanece fixo independentemente do tipo carregamento aplicado, ou seja, o módulo elastoplástico do material será nulo. (Souza Neto, et al, 2008)

A Figura 4 mostra a curva tensão $(\sigma)$ - deformação $(\varepsilon)$ de um carregamento cíclico uniaxial (tensão-compressão) em uma situação perfeitamente plástica.

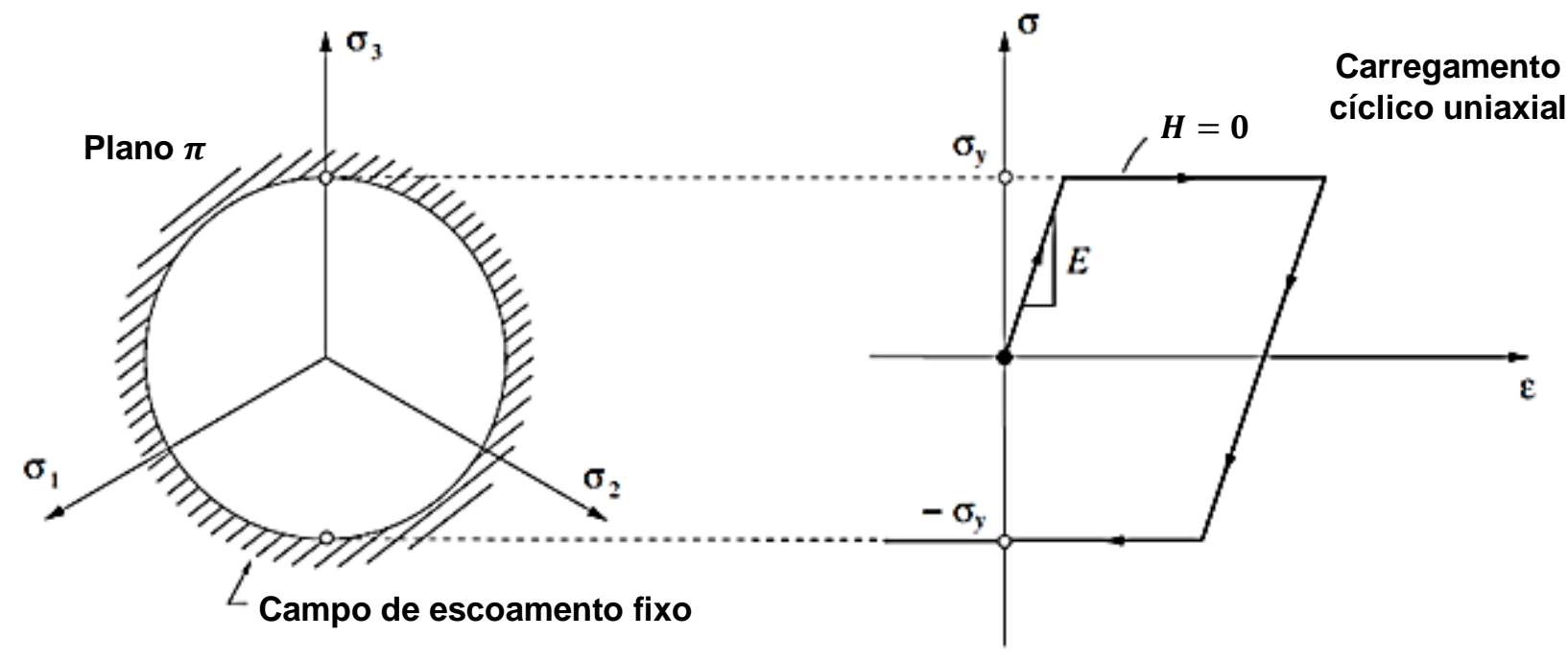

Figura 4. Plasticidade ideal. Fonte: Adaptado Souza Neto, el al, 2008. 
Neste caso, $H$ é o coeficiente de endurecimento, $E$ é o módulo de Young do material, $\sigma_{1}, \sigma_{2}$ e $\sigma_{3}$ é o espaço das tensões principais no plano $\pi$ e $\sigma_{y}$ representa o limite de escoamento. Observa-se que, para plasticidade ideal, o coeficiente de endurecimento cinemático se mantém nulo, não havendo evolução do campo de escoamento inicial, independentemente da deformação aplicada.

\subsubsection{Endurecimento isotrópico}

O endurecimento isotrópico se dá quando a evolução da superfície de escoamento é, em qualquer estado de endurecimento, correspondente a de expansão da superfície de escoamento inicial, sem que haja sua translação. O modelo uniaxial descrito na Figura 3 é um exemplo típico de um modelo de endurecimento isotrópico.

Nesse modelo, ilustrado na Figura 5, o domínio elástico expande igualmente em tensão e compressão durante o fluxo plástico e, para um modelo de plasticidade multiaxial com uma superfície de von Mises, o endurecimento isotrópico corresponde ao aumento do raio do cilindro do espaço das tensões principais. (Souza Neto, et al, 2008)

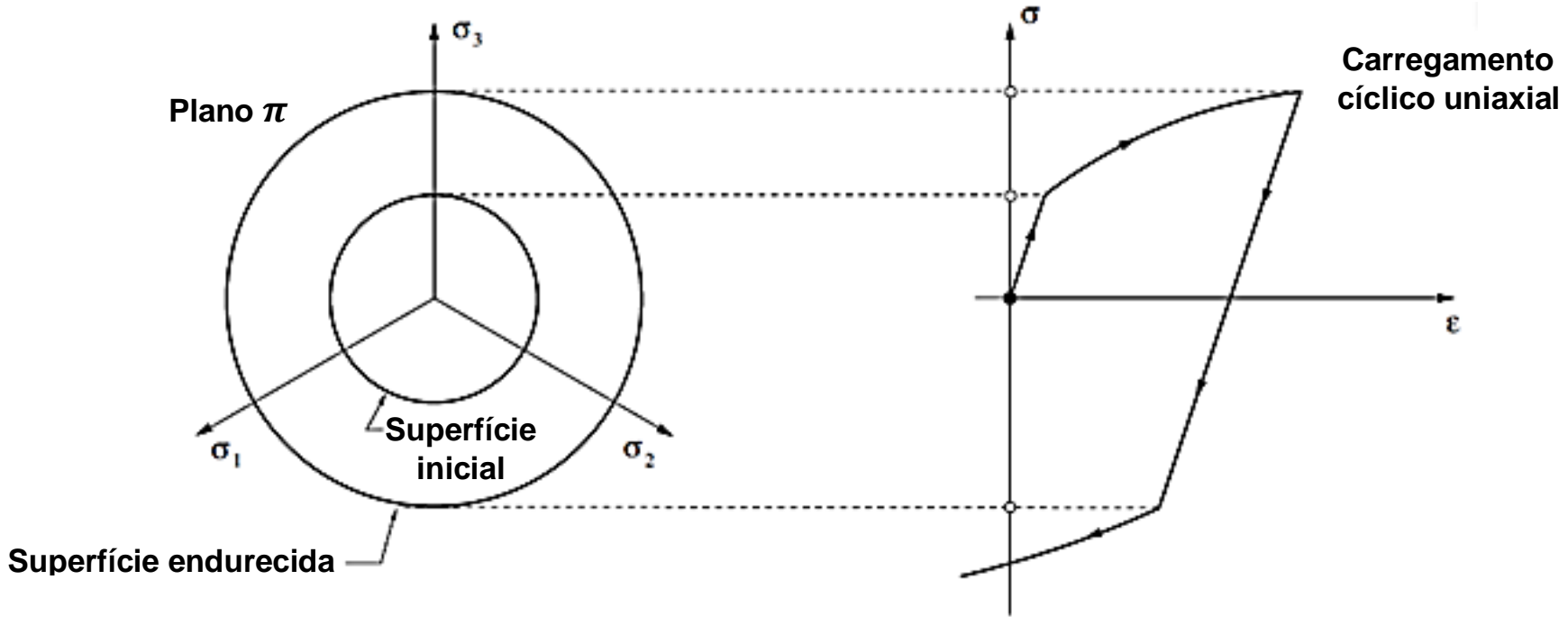

Figura 5. Endurecimento isotrópico não linear. Fonte: Adaptado Souza Neto, el al, 2008. 


\subsubsection{Endurecimento cinemático}

O endurecimento cinemático é caracterizado pela presença do efeito de Bauschinger e, a fim de proporcionar um meio simples de introduzi-lo para as relações tensão-deformação da teoria da plasticidade. Prager W. (1956), introduziu um modelo cinemático no qual a faixa elástica total é mantida constante e o envelope de falha de von Misses tem sua superfície de escoamento inicial transladada, sem deformá-la.

Armnstrong (1966) observou que o chamado de Efeito Bauschinger se torna muito visível em situações relativas à aplicação de regimes de carregamentos cíclicos, pois a reversão do carregamento influencia o limite de escoamento na direção oposta e faz com que a superfície de escoamento esteja constantemente se transladando.

A Figura 6 ilustra o efeito do endurecimento cinemático não linear sobre a superfície de escoamento, e sobre a curva tensão-deformação em um ensaio uniaxial cíclico. Nela, $\sigma_{y}$ representa o limite de escoamento do material e a variável $\boldsymbol{\beta}$ é o símbolo que representa o tensor das tensões cinemáticas e significa a translação da superfície de escoamento em relação ao ponto inicial do domínio elástico no espaço das tensões desviadoras, ele também pode ser chamado de tensão de retorno.

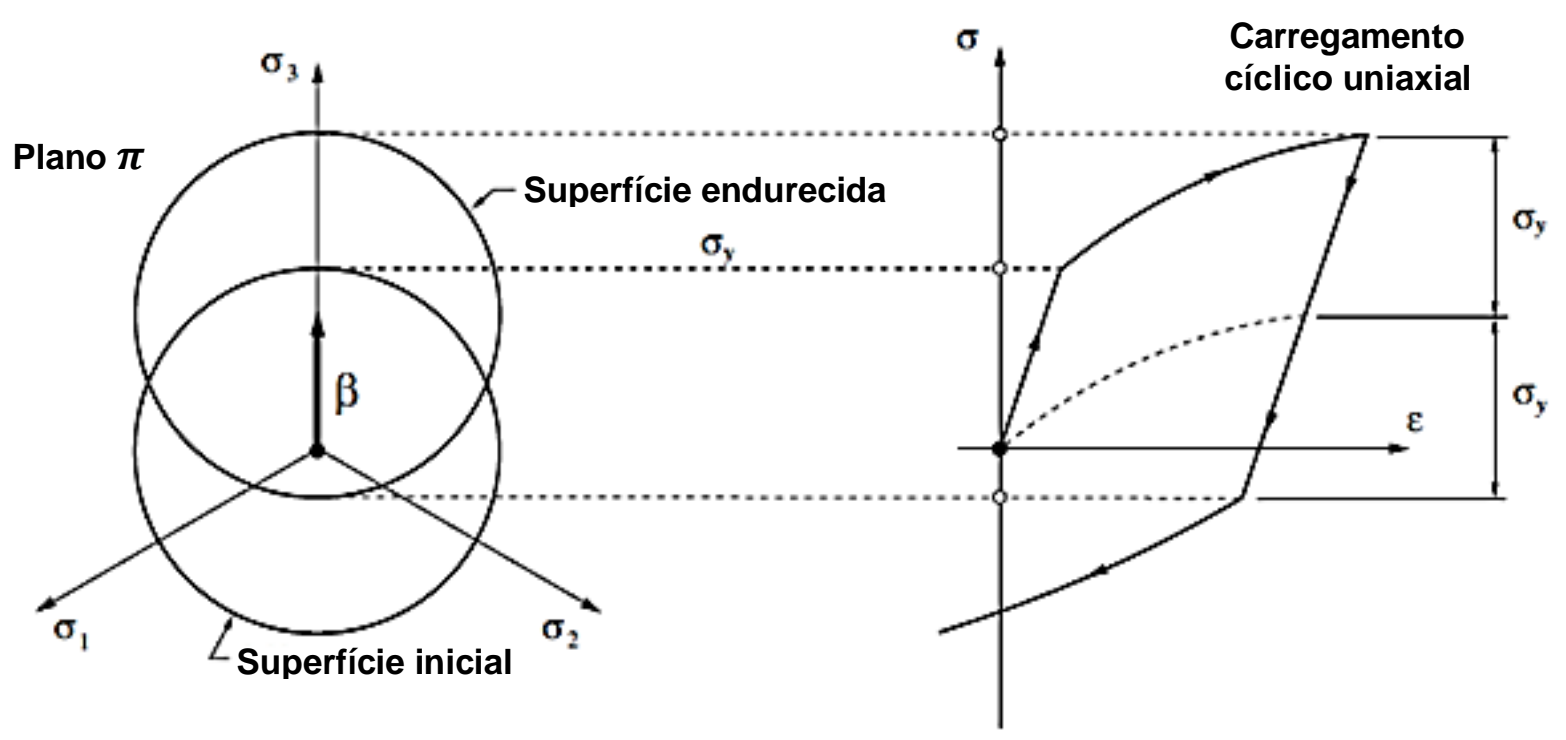

Figura 6. Endurecimento cinemático não linear. Fonte: Adaptado Souza Neto, el al, 2008. 
Dos tipos de endurecimentos apresentados nos tópicos anteriores, o presente trabalho optou pela utilização de uma lei com capacidade de previsão da evolução do endurecimento cinemático não linear, uma vez que o estudo em pauta tem relação com casos de previsão de vida a fadiga, os quais são exemplos clássicos de análises em plasticidade cíclica. O subitem 2.2. apresentará os modelos disponíveis para previsão da evolução do tensor das tensões cinemáticas.

\subsection{MODELO PARA PLASTICIDADE CÍCLICA}

Conforme abordado, a plasticidade cíclica é um fenômeno de interesse prático em diversos projetos, onde muitos componentes são analisados a fim de se estabelecer as margens de falha por fadiga, as quais podem ocorrer devido a um maior número de ciclos de carga do que o previsto para vida do material. (Jiang, Y. \& Zhang, J., 2008)

\subsubsection{Prager Linear}

Visando completar a definição dos modelos de plasticidade com endurecimento cinemático, equações de evolução para $\boldsymbol{\beta}$ são obrigatórias. Assim Prager (1956) propôs uma das leis de endurecimento cinemático mais simples usadas para descrever a resposta plástica dos materiais submetidos a carregamentos cíclicos.

Conhecida como Prager Linear, é a lei mais utilizada para o mapeamento de endurecimentos de cinemáticos, na qual a equação de evolução da taxa tensor das tensões cinemáticas $(\boldsymbol{\beta})$ é dada por uma definição do modelo de plasticidade de endurecimento cinemático que se baseia em uma relação linear entre a taxa de variação de $\boldsymbol{\beta}$ e a lei de fluxo plástico. A lei de endurecimento cinemático de Prager é definida por:

$$
\dot{\boldsymbol{\beta}}=\frac{2}{3} H^{k} \dot{\boldsymbol{\varepsilon}}^{p}
$$

onde $H^{k}$ é o coeficiente de endurecimento cinemático, $\dot{\boldsymbol{\varepsilon}}^{p}$ é o tensor da lei de fluxo plástico e $\dot{\boldsymbol{\beta}}$ é a taxa de evolução do tensor das tensões cinemáticas. 
Nota-se que a linearidade da relação tensão-deformação obtida pela lei de Prager é o principal motivo de críticas ao uso desse modelo, pois, apesar de descrever de maneira adequada alguns aspectos do efeito de Bauschinger, o modelo ainda é bastante falho na convergência com os resultados experimentais.

Isso ocorre porque apesar do modelo proposto por Prager reproduzir de forma matematicamente satisfatória o efeito Bauschinger, este não descreve bem os carregamentos que envolvem cargas e descargas subsequentes em direções reversas (Chaboche, 1986). Além de, devido a sua simplicidade linear, deixar muito a desejar com relação à sua capacidade preditiva em reproduzir a parte inicial não linear da curva de histerese experimental, como apresentado na Figura 7.

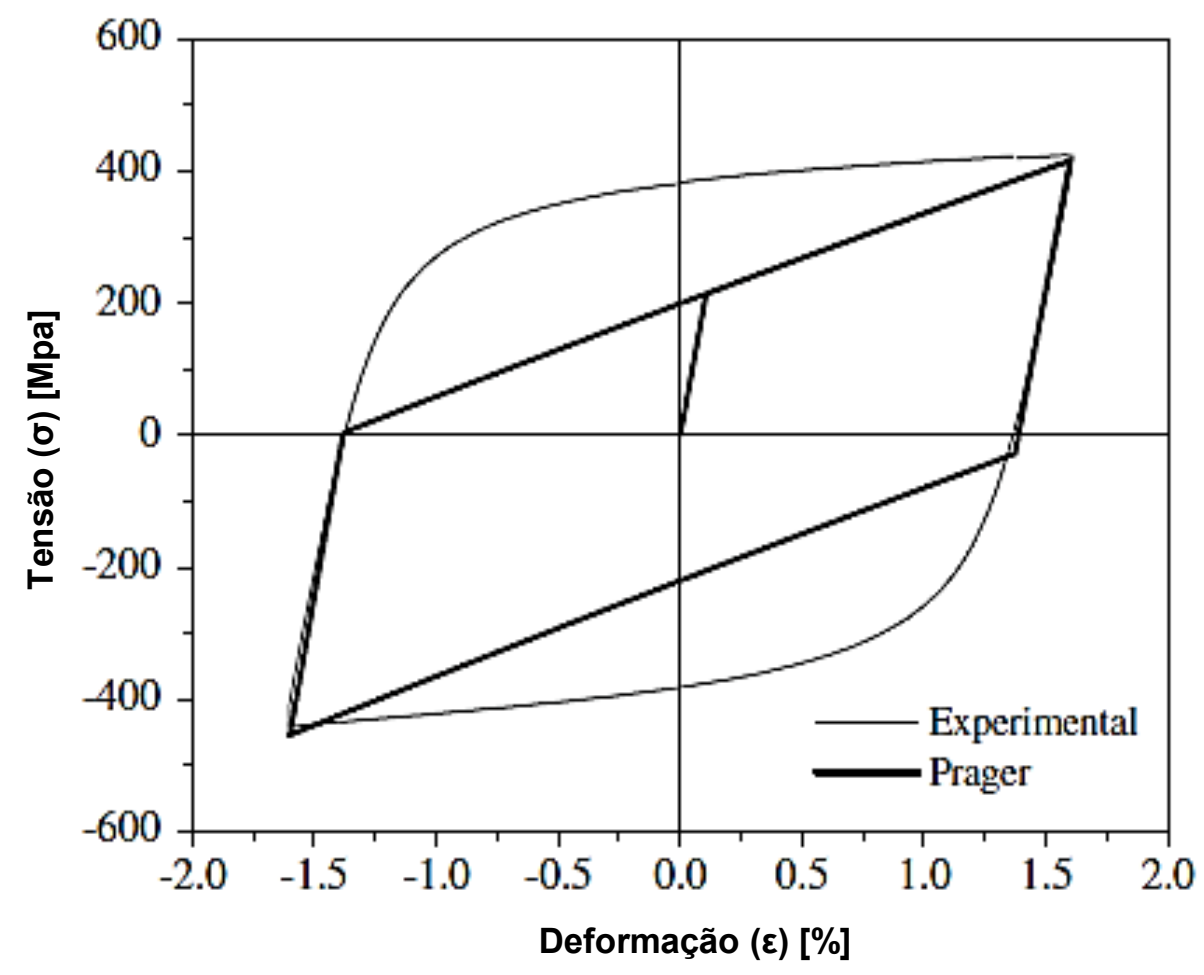

Figura 7. Exemplo de curva tensão-deformação para o modelo de Prager. Diferença entre o laço de histerese experimental e numérico. Fonte: Adaptado de Paul, S.K., et al, 2010. 


\subsubsection{Armstrong-Frederick}

A partir do modelo de Prager, Armstrong e Frederick (1966), propuseram a introdução de um termo de saturação não linear a equação anteriormente apresentada. Este tinha como intuito descrever de maneira mais apropriada a curva tensãodeformação em carregamentos cíclicos.

Chaboche (1986) destaca que a utilização do modelo não linear de ArmstrongFrederick não corrige apenas a forma do laço de histerese, mas também aspectos relativos à estabilização do ciclo, como o fornecimento de relações entre as amplitudes do ciclo estabilizado. Assim, a lei de endurecimento cinemático de Armstrong-Frederick pode ser definida como sendo:

$$
\dot{\boldsymbol{\beta}}=\frac{2}{3} H^{k} \dot{\boldsymbol{\varepsilon}}^{p}-b \dot{\bar{\varepsilon}} \boldsymbol{\beta}^{\beta},
$$

onde $H^{k}$ é o coeficiente de endurecimento cinemático, $\dot{\boldsymbol{\varepsilon}}^{p}$ é a lei de fluxo plástico, $b$ é um parâmetro material, $\dot{\bar{\varepsilon}}^{p}$ é a deformação equivalente, $\boldsymbol{\beta}$ tensor das tensões cinemáticas e $\dot{\boldsymbol{\beta}}$ é a taxa de evolução do tensor das tensões cinemáticas.

Observa-se que ao introduzir esta lei de endurecimento cinemático ao critério de escoamento de von Mises a saturação corresponde a um valor máximo para o módulo de $\boldsymbol{\beta}$, a partir do qual o material passa a se comportar como perfeitamente plástico (Souza Neto, et al, 2008).

A Figura 8 compara curva tensão-deformação do modelo de Armstrong e Frederick com um laço de histerese estável obtido a partir de resultados experimentais (Paul, S.K., et al, 2010). Essa figura permite observar que apesar da considerável melhora do ajuste da curva tensão-deformação, em relação ao modelo de Prager, a utilização de apenas uma equação exponencial não é capaz de representar de maneira adequada a curva experimental. Note que o aumento do valor de $H^{k}$ melhoraria o ajuste no início da deformação plástica, mas prejudicaria sua trajetória ao longo da evolução da deformação. 
Uma desvantagem do modelo de Armstrong e Frederick é que apesar da capacidade de representar com boa aproximação a condição de plasticidade cíclica de materiais, ele ainda não é suficientemente robusto para prever a resposta convirja a curva de histerese do material. Por esta razão, várias modificações do modelo de Armstrong e Frederick têm sido propostas numa tentativa de melhorar esse tipo de resposta. (Paul, S.K., et al, 2010)

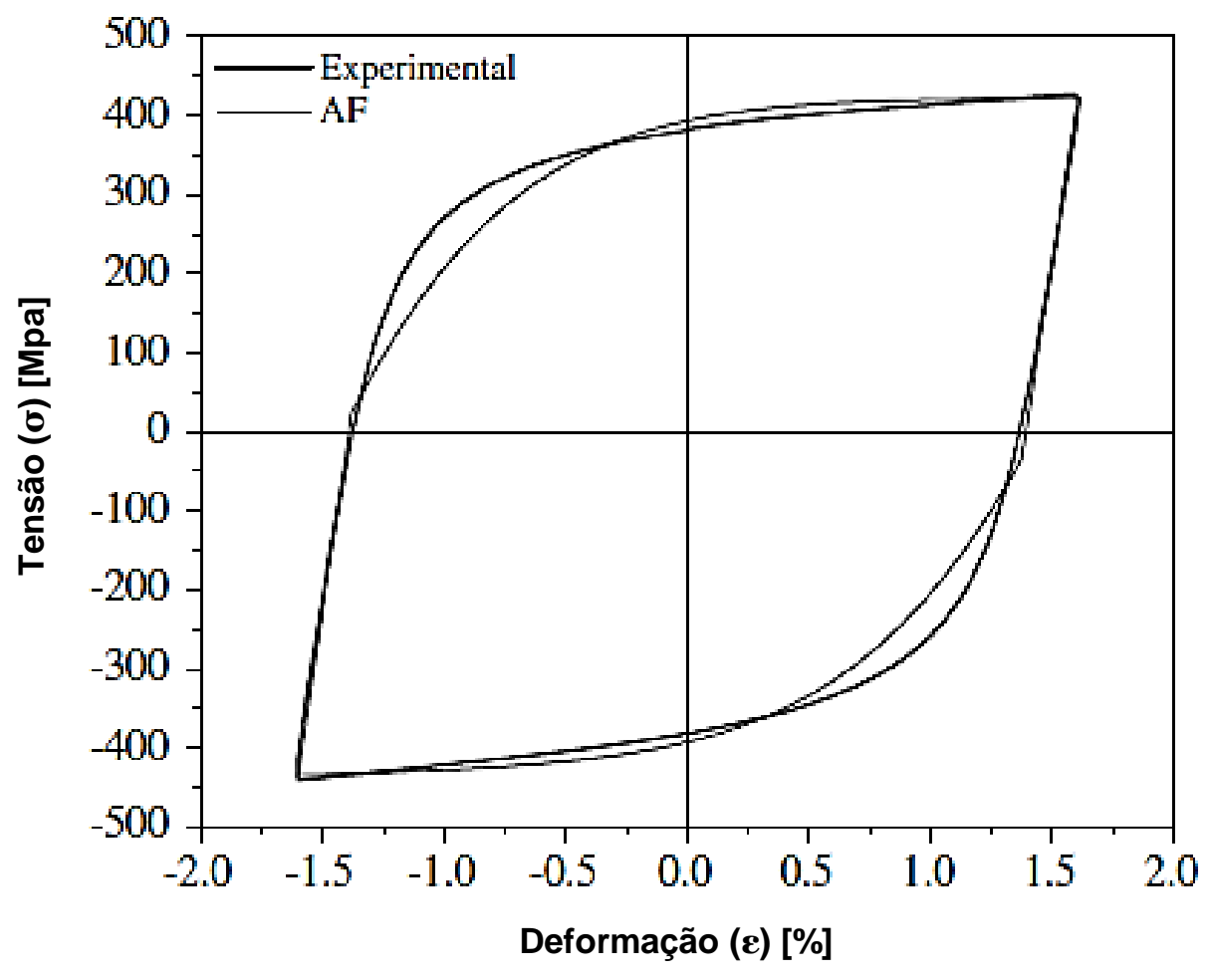

Figura 8. Exemplo de curva tensão-deformação para o modelo de Armstrong e Frederick. Diferença entre o laço de histerese experimental e numérico. Fonte: Adaptado de Paul, S.K., et al, 2010.

\subsubsection{Chaboche}

O modelo de endurecimento cinemático proposto por Chaboche (1986) pode ser considerado como uma superposição de funções do modelo de Armstrong e Frederick (AF), de forma que Chaboche se torna idêntico ao modelo $A F \operatorname{com} n=1$. (Dahlberg M. e Segle P., 2010) 
Matematicamente, o modelo de Chaboche apresenta-se em sua forma generalizada e em função de "n" número de termos, conforme segue:

$$
\dot{\boldsymbol{\beta}}=\sum_{i=1}^{n}\left(\frac{2}{3} H_{i}^{k} \dot{\boldsymbol{\varepsilon}^{p}}-b_{i} \dot{\bar{\varepsilon}}^{p} \boldsymbol{\beta}_{i}\right)
$$

onde $H_{i}^{k}$ é o coeficiente de endurecimento cinemático, $\dot{\boldsymbol{\varepsilon}}^{p}$ é a lei de fluxo plástico, $b_{i}$ é um parâmetro material, $\dot{\varepsilon}^{p}$ é a deformação equivalente, $\boldsymbol{\beta}_{\boldsymbol{i}}$ é o tensor das tensões cinemáticas e $\dot{\boldsymbol{\beta}}$ é a taxa de evolução do tensor das tensões cinemáticas.

A análise desse modelo demonstra proporciona uma maior flexibilidade ao ajuste dos parâmetros materiais $H_{i}^{k}$ e $b_{i}$, mantendo assim vantagens em relação ao modelo de $\mathrm{AF}$, inclusive a não linearidade, permitindo a parametrização do material de forma a mapear a evolução da curva de histerese em precisão razoavelmente mais estável.

Contudo, a introdução do somatório citado faz com que sejam necessárias mais informações a respeito do material, ou seja, enquanto no modelo linear de Prager a única constante de material presente era o módulo de endurecimento cinemático $H^{k}$. No modelo de AF houve a introdução de uma nova constante material $b$, ou seja, passando a ser necessário de calibração de $b$ e $H^{k}$, ou seja, 2 (dois) pontos de calibação. (Paul, S.K., et al, 2010)

Desta forma, o modelo de Chaboche sendo uma extensão de AF, haverá $2 n$ termos a serem calibrados. Geralmente, fazendo-se $n=3$ já é possível uma boa correlação do modelo com os dados experimentais, neste caso, seria necessário calibrar o modelo para 6 constantes materiais. (Chaboche, 1986).

A Figura 9 compara a curva tensão-deformação do modelo de Chaboche com um laço de histerese estável obtido a partir de resultados experimentais. (Paul, S.K., et al, 2010) 


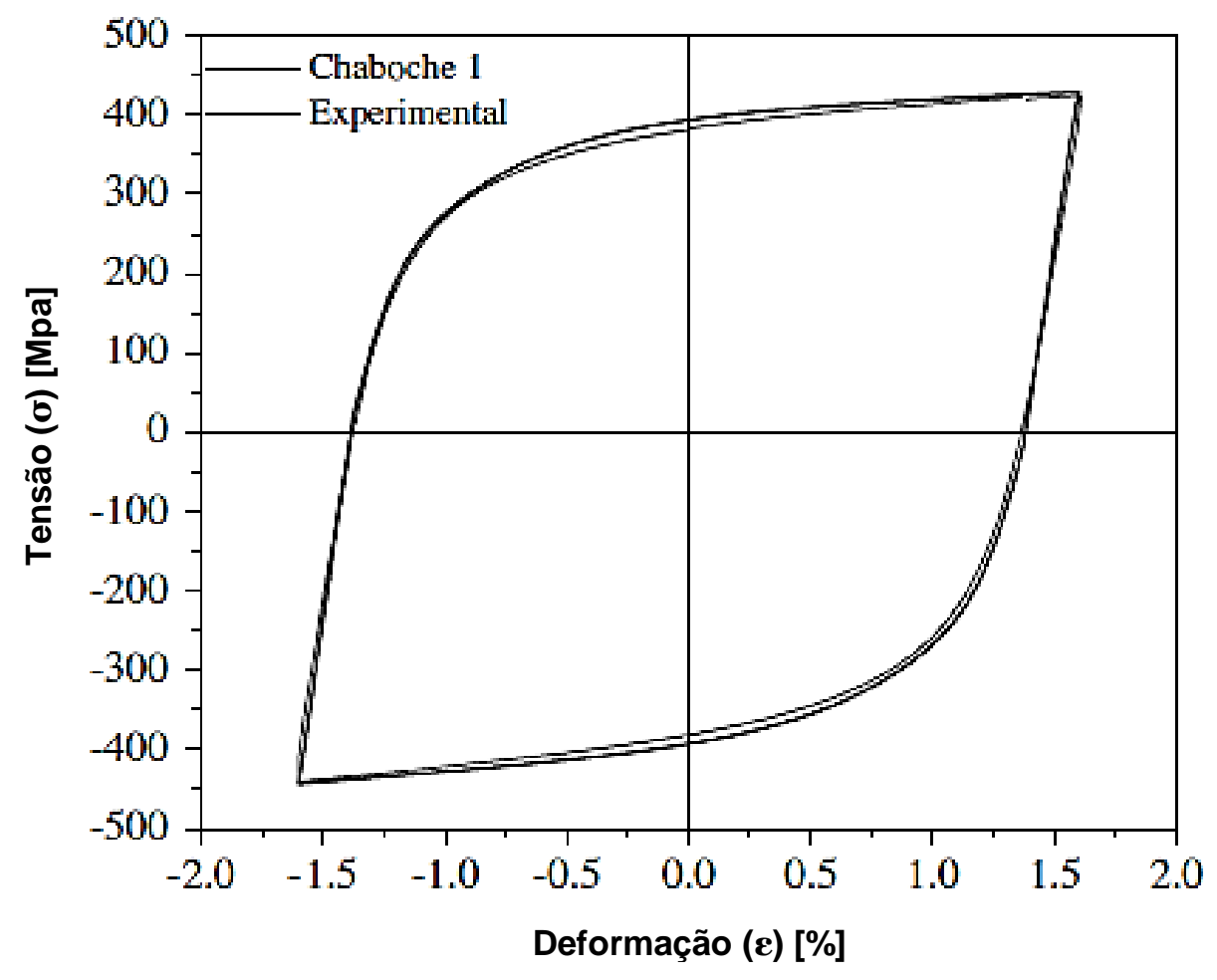

Figura 9. Exemplo de curva tensão-deformação para o modelo de Chaboche. Diferença entre o laço de histerese experimental e numérico. Fonte: Adaptado de Paul, S.K., et al, 2010.

Uma desvantagem do modelo de Chaboche é o fato dele apresentar pouca sensibilidade aos efeitos de carregamentos não proporcionais, sendo está uma característica, também, dos modelos de Prager e AF (Bari e Hassan, 2000).

Por fim, visando à delimitação da lei de plasticidade cíclica a ser empregada neste trabalho, expõe-se que apesar de lei de endurecimento cinemático de Chaboche apresentar considerável melhora na aproximação dos laços de histerese experimentais e numéricos, sua semelhança com AF faz com que uma futura ampliação do modelo se torne demasiadamente simples. Dessa forma, estabelecendo o foco na integração de um modelo baseado na micromecânica de defeitos a uma lei de previsão da taxa de evolução do tensor das tensões cinemáticas, opta-se por seguir com a utilização do critério elaborado por Armstrong e Frederick (1966). 


\subsection{MODELOS BASEADOS NA MICROMECÂNICA DE DEFEITOS}

\subsubsection{Gurson}

Um dos primeiros modelos baseados na micromecânica de defeitos para descrever a fratura dúctil é o de Gurson (1977), onde este introduziu uma relação entre o parâmetro de dano e a deformação plástica quando o material está sob uma deformação finita. $O$ trabalho de Gurson parte de uma extensão do modelo de von Mises e descreve o crescimento dos vazios baseado no desenvolvimento de Rice e Tracey (1969), que analisaram um vazio dentro de uma matriz metálica. (Souza Neto, et al, 2008)

Segundo Gurson (1977), para o aparecimento de micro vazios associados a grandes deformações plásticas, bem como a degradação interna para se encontrar as equações de evolução do modelo, era necessário assumir um vazio na forma esférica contido em uma matriz de material ou volume do elemento representativo, conforme ilustrado na Figura 10.

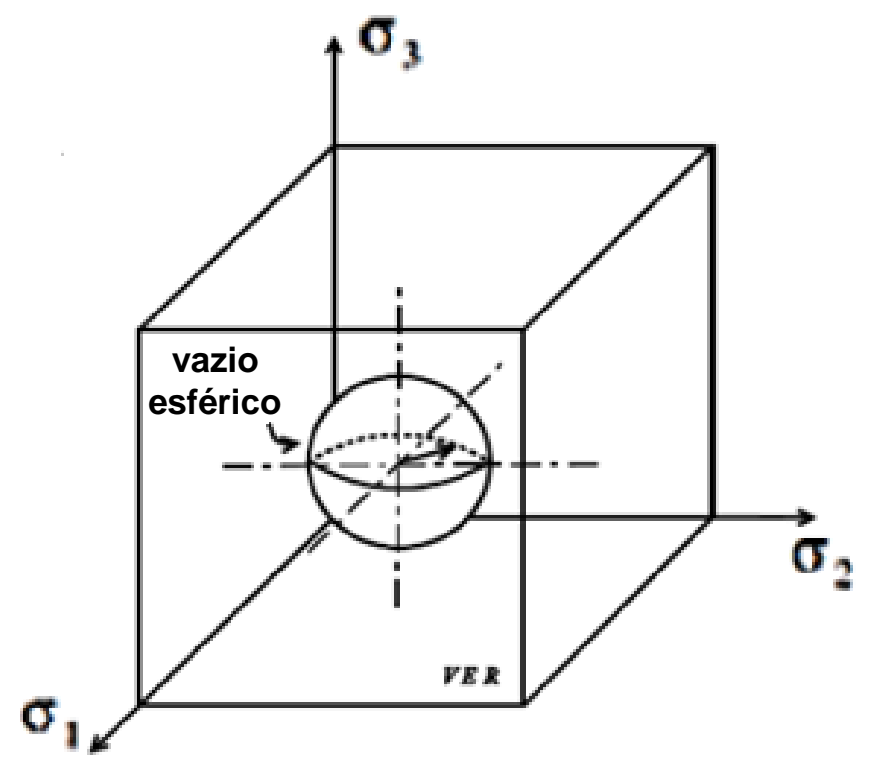

Figura 10. Representação esquemática do elemento representativo com um vazio esfério. Adaptado de Gurson, 1977. 
Onde, através da relação entre o volume do vazio $\left(V_{\text {vazio }}\right)$ e o volume do elemento representativo $\left(V_{E R}\right)$, é dada a fração volumétrica do vazio do material como sendo:

$$
f=\frac{V_{v a z i o}}{V_{E R}}
$$

Em sequência, para carregamentos predominantemente de tração, a Figura 11 apresenta a relação entre a evolução do carregamento macroscópico e a degradação da microestrutura do material, a qual acontece pela existência de micro vazios ou pela nucleação de novos.

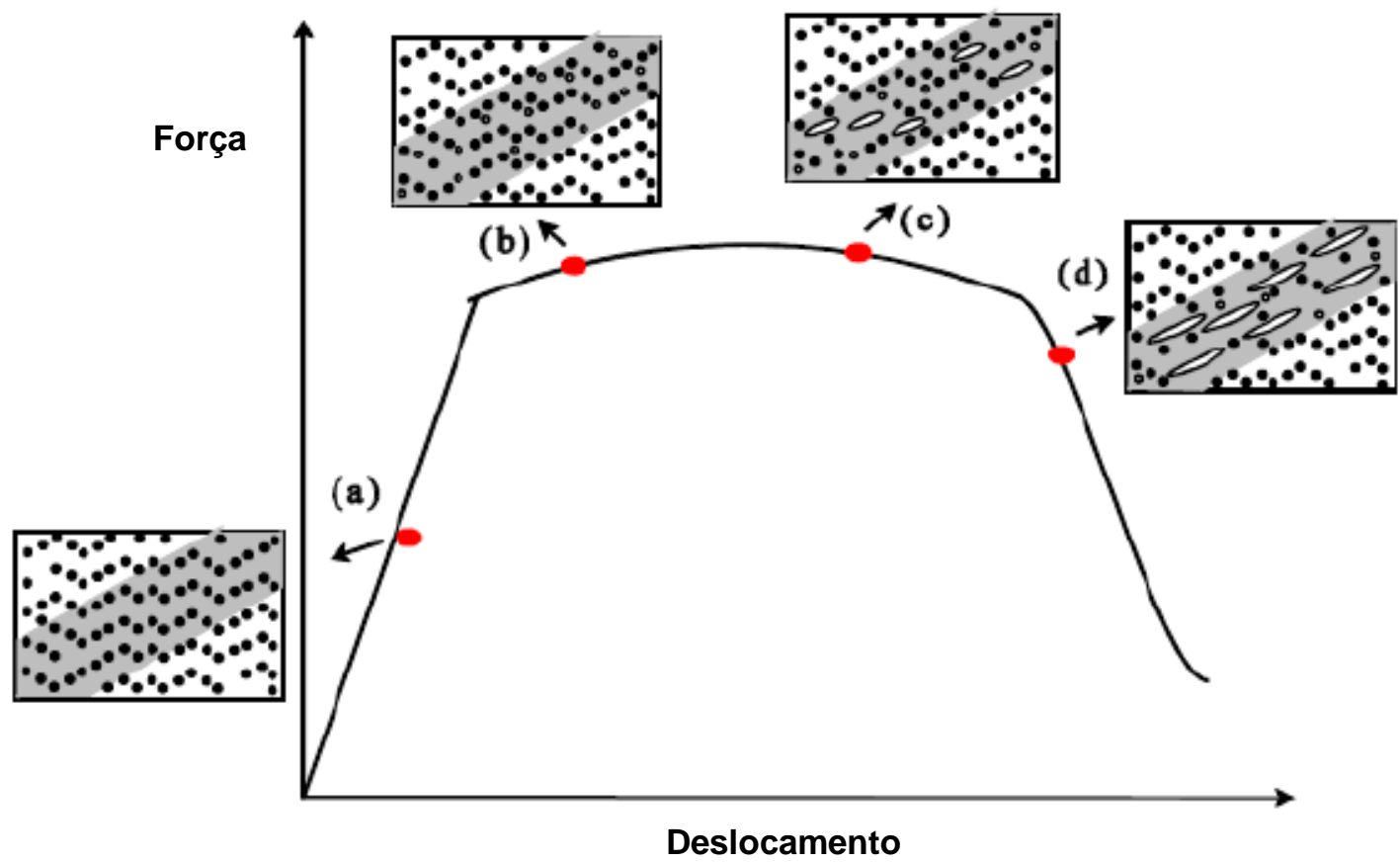

Figura 11. Representação esquemática do processo de nucleação, crescimento e coalescência dos micros vazios e a relação com o carregamento macroscópico. Fonte: Pineau e Pardoen, 2003.

De forma que cada estágio representa: (a) o domínio elástico, não havendo alteração na microestrutura; (b) que o aumento da carga macroscópica provoca deformação plástica localizada e induz a nucleação de vazios; (c) o aumento do crescimento de vazios devido às altas tensões hidrostáticas; e, (d) após o crescimento (fase c), a coalescência dos vazios. 
A Figura 12 ilustra através de uma foto em escala manométrica o processo esquematizado na Figura 11.

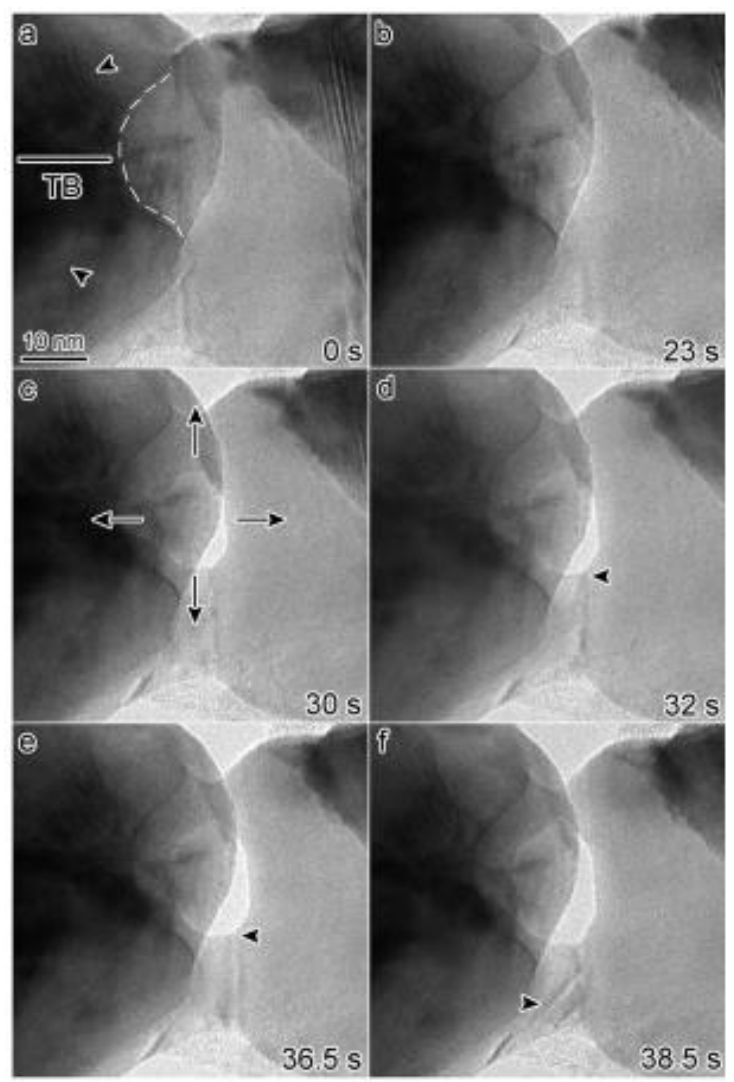

Figura 12. Imagem ótica da nucleação de um vazio em escala manométrica. Zheng, H., et al. (2014).

Assim, observa-se que durante a deformação do corpo há um aumento da fração volumétrica de vazios prevista pelo modelo de Gurson e, assumindo a incompressibilidade plástica do mesmo, a densidade do elemento representativo de um material com vazios pode ser descrita da seguinte forma:

$$
\rho=\rho_{m} \frac{V_{m}}{V_{E R}}
$$

onde, $\rho$ é densidade do elemento representativo, $\rho_{m}$ é a densidade da matriz de material, $V_{m}$ é o volume da matriz de material e $V_{E R}$ é o volume do elemento representativo.

Logo, a relação entre o volume da matriz de material $(\mathrm{Vm})$ e a fração volumétrica de vazios $(f)$, manipulando as equações 2.3.1. e 2.3.2., pode ser definida por: 


$$
\frac{V_{m}}{V_{E R}}=(1-f)
$$

e, substituindo a equação 2.3.3 na 2.3.1, temos:

$$
\rho=\rho_{m}(1-f),
$$

logo, a taxa da densidade do elemento representativo $(\dot{\rho})$, será determinada pela derivada da equação 2.3.4. no tempo, onde $\rho_{m}$ representa a taxa da densidade da matriz do material e $\dot{f}$ a taxa da fração volumétrica de vazios.

$$
\dot{\rho}=\dot{\rho}_{m}(1-f)-\rho_{m} \dot{f}
$$

Assumindo a matriz de material como plasticamente incompressível, a tensão volumétrica elástica sendo presumidamente descartada e observando-se que o princípio de conservação de massa diz que $\dot{\rho}_{m}=0$, após algumas manipulações algébricas, obtém-se que:

$$
\dot{f}=-\frac{\dot{\rho}}{\rho_{m}}=-\frac{\dot{\rho}}{\rho}(1-f)
$$

Pelo princípio da conservação de massas, temos que a taxa de deformação volumétrica será determinada por:

$$
-\frac{\dot{\rho}}{\rho}=\dot{\varepsilon}_{v}=\dot{\varepsilon}_{v}^{e}+\dot{\varepsilon}_{v}^{p}
$$

onde as taxas de deformações volumétricas elástica e plástica são representadas, respectivamente, por $\dot{\varepsilon}_{v}^{e}$ e $\varepsilon_{v}^{p}$. No modelo de Gurson a matriz de material é assumida como sendo rígido-plástica, portanto, desconsiderando a parte elástica, se pode reescreve-la, a partir da equação 2.3.6., como:

$$
\begin{gathered}
\dot{f}=-\frac{\dot{\rho}}{\rho_{m}} \\
\dot{f}=\dot{\varepsilon}_{v}^{p}(1-f)
\end{gathered}
$$


A equação 2.3.9 representa a contribuição mais significante para a degradação de um material poroso e expressa a lei de evolução para fração volumétrica de vazios.

Partindo da teoria apresentada, promoveu-se a apresentação da função de escoamento originalmente determinada por Gurson, 1977, onde, para uma matriz de vazios agregados, foi expresso que:

$$
\phi=J_{2}-\frac{1}{3}\left[1+f^{2}-2 f \cosh \left(\frac{3 p}{2 \sigma_{y}}\right)\right] \sigma_{y}^{2},
$$

onde $J_{2}$ é o segundo invariante do tensor tensão desviador, $\rho$ é a pressão hidrostática (primeiro invariante do tensor tensão), $f$ é a fração volumétrica de vazios e $\sigma_{y}$ o limite de escoamento do material.

Uma característica do modelo original de Gurson (1977) é sua limitação quando a captura dos efeitos de distorção e cisalhamento, uma vez que sua aplicação considera um único parâmetro de dano, a porosidade $f$, como a fração volumétrica de vazios. Como consequência, em uma situação onde o carregamento do corpo prever componentes cisalhantes, o modelo não irá prever a distorção do vazio por partes destas forças e apresentará uma evolução falha da porosidade.

Observa-se ainda que tornando a fração volumétrica de vazios inicial nula, $f_{o}=$ 0, o modelo de Gurson reduz-se ao modelo de von Mises, não apresentando evolução da porosidade efetiva ao longo do crescimento da deformação plástica equivalente. Contudo, para situações em que a fração volumétrica de vazios inicial for positiva, $\mathrm{f}_{\mathrm{o}}>0$, haverá um aumento exponencial da porosidade em função da evolução da deformação plástica equivalente do corpo. A Figura 13 ilustra essa definição. 


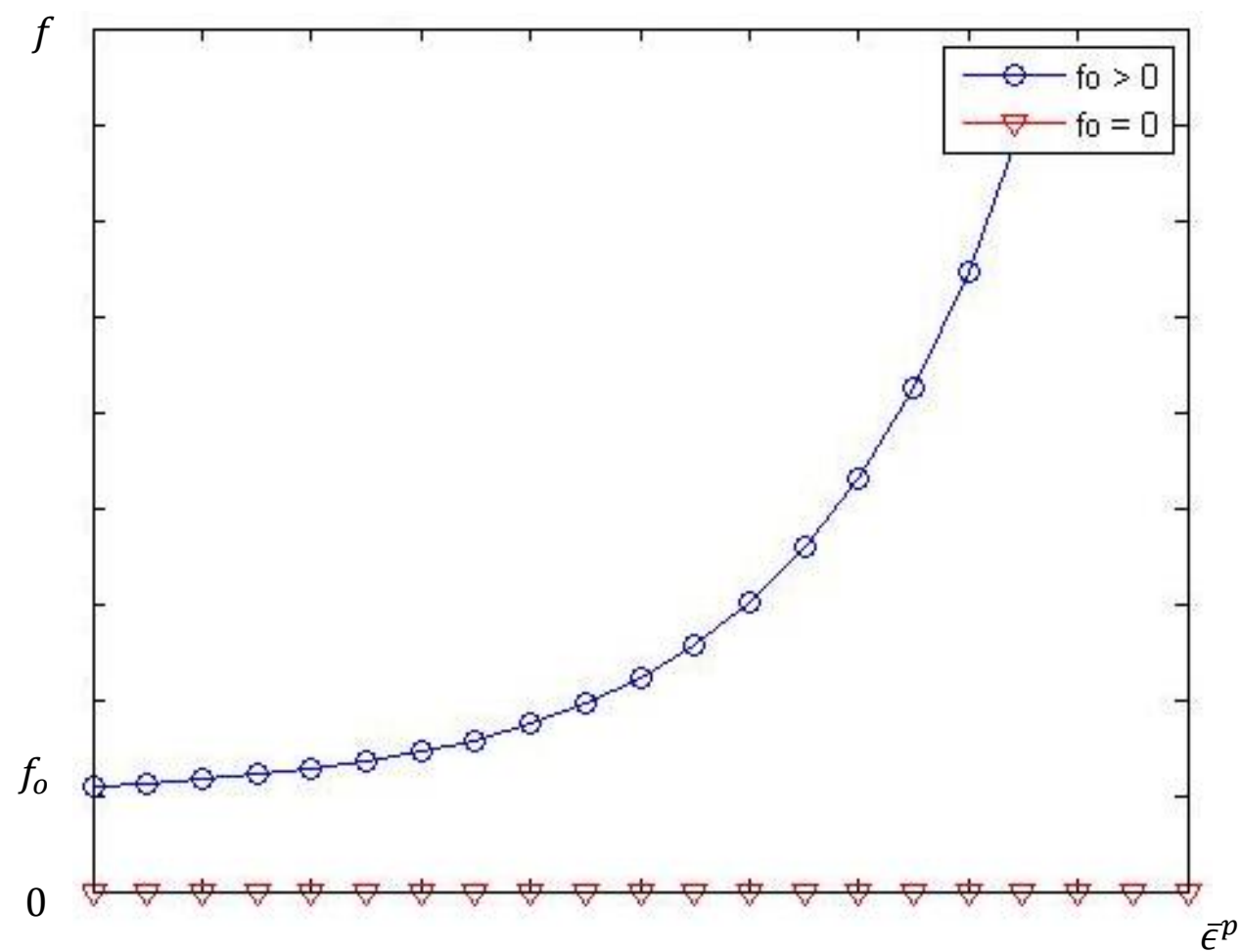

Figura 13. Gráfico comparativo da evolução da porosidade efetiva em função da porosidade inicial $\left(f_{o}=\right.$ o ou $f_{o}>0$ ), e da deformação plástica equivalente.

Dessa forma, segundo Gurson (1977), pode-se afirmar que a degradação das propriedades mecânicas de qualquer material é função dos micros vazios pré-existentes ou que venham a se formar na matriz desse material.

\subsubsection{Gurson-Tvergaard-Needleman (modelo GTN)}

O modelo original de Gurson traz em si uma deficiência percebida onde, em qualquer que seja a tensão, não é possível prever qualquer evolução da fratura se a porosidade inicial for zero. Dessa forma, uma das mais conhecidas modificações proposta para aprimorar o citado modelo foi apresentada por Chu \& Needleman (1980) e mais tarde utilizado por Tvergaard (1984), em um modelo em que havia a introdução do histórico das tensões e que passou a ser conhecido como Gurson-Tvergaard- Needleman (GTN). 
A Figura 14 ilustra a evolução da porosidade efetiva para cada um dos modelos citados.

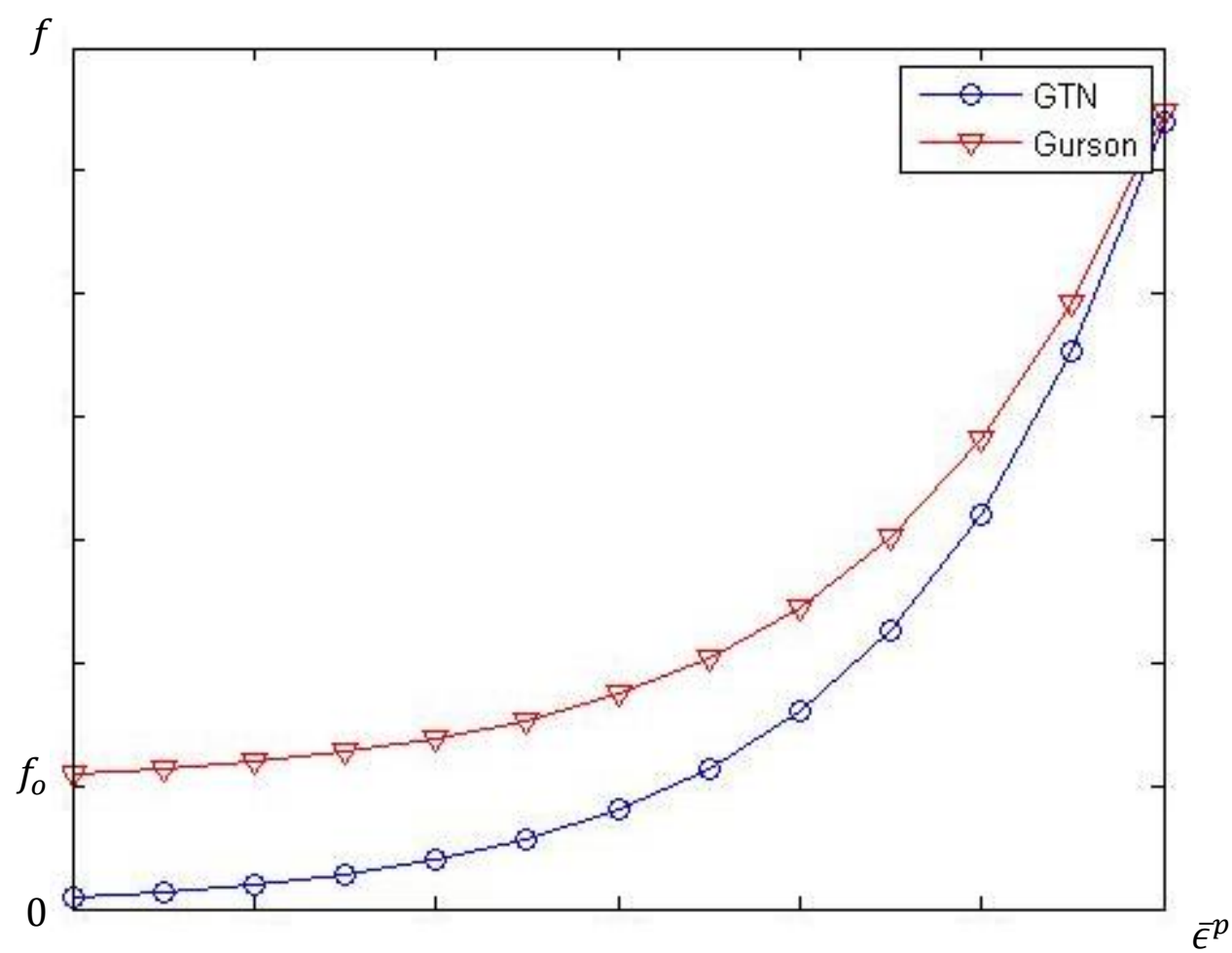

Figura 14. Gráfico comparativo da evolução da porosidade efetiva nos modelos de Gurson e GTN.

Nesse modelo, a evolução do dano é representada por três mecanismos simultâneos ou sucessivos: nucleação, crescimento e coalescência de vazios, sendo a porosidade efetiva $\left(f^{*}\right)$ introduzida e calculada através da equação bi-linear apresentada na equação 2.3.11.

$$
f^{*}=\left\{\begin{aligned}
f, & f<f_{c} \\
f_{c}+\left(\frac{1}{q_{1}}-f_{c}\right) \frac{\left(f-f_{c}\right)}{\left(f_{f}-f_{c}\right)}, & f \geq f c,
\end{aligned}\right.
$$

onde $f_{c}$ é o volume crítico do vazio, $f_{f}$ é o volume do vazio na fratura e $q_{n}$ são os parâmetros de ajuste numérico.

Assim, a equação 2.3.11 apresenta que para situações em que a porosidade é inferior ao valor crítico $f_{c}$, está passa a ser definida pela contribuição dos mecanismos de nucleação e crescimento, enquanto que para situações em que $f \geq f c$, o mecanismo de coalescência é ativado e passa a contribuir com a porosidade efetiva. 
Destaca-se que no modelo GTN a taxa de crescimento da porosidade passa a ser o somatório da taxa de nucleação com a taxa de crescimento de vazios.

$$
\dot{f}=\dot{f}^{n}+\dot{f}^{g},
$$

onde, $\dot{f}^{n}$ é a taxa de nucleação do vazio e $\dot{f}^{g}$ é a taxa de crescimento de vazios.

Ou seja, para descrever o mecanismo de nucleação por deformação plástica ou pela tensão hidrostática, GTN formularam a definição do mecanismo de nucleação com base na deformação plástica equivalente, conforme equação 2.3.13.

$$
\dot{f}^{n}=\frac{f_{N}}{S_{N} \sqrt{2 \pi}} \exp \left[-\frac{1}{2}\left(\frac{\bar{\varepsilon}^{p}-\varepsilon_{N}}{S_{N}}\right)^{2}\right] \dot{\bar{\varepsilon}}^{p},
$$

onde, $f_{N}$ é o volume das frações de todas as partículas de vazios com potencial para nuclear, $\varepsilon_{N}$ é a deformação média para nucleação, $S_{N}$ é o desvio padrão para deformação média para nucleação e $\dot{\bar{\varepsilon}}^{p}$ é a taxa de deformação plástica equivalente.

Concluindo, a partir das novas definições postas por GTN, que a função de escoamento original de Gurson, 1977, pode ser reescrita como:

$$
\phi=J_{2}-\frac{1}{3}\left[1+q_{3} f^{* 2}-2 q_{1} f^{*} \cosh \left(\frac{q_{2} 3 p}{2 \sigma_{y}}\right)\right] \sigma_{y}^{2}
$$

Apresentado o modelo GTN e conhecidas suas vantagens em relação ao modelo original de Gurson, 1977, opta-se pela escolha do modelo clássico para continuidade da formulação do presente trabalho, uma vez que este necessita uma quantidade menor de parâmetros materiais a serem calibrados, neste caso, somente a porosidade inicial $f_{o}$.

A partir do Capítulo 3, o modelo de micromecânica de defeitos baseado no critério de Gurson será desenvolvido acoplado à lei de endurecimento cinemático de ArmstrongFrederick. 


\section{DESENVOLVIMENTO DO MODELO ELASTO-PLÁSTICO DE GURSON COM ENDURECIMENTO CINEMÁTICO}

Neste capítulo será desenvolvido o modelo elastoplástico proposto por Gurson com endurecimento cinemático, sendo adotada a lei de Armstrong \& Frederick para evolução do tensor das tensões cinemáticas, $\boldsymbol{\beta}$.

\subsection{DESENVOLVIMENTO DO MODELO MATEMÁTICO}

Definidas a lei elástica, expressa pela lei de Hooke generalizada, equação 2.1.1 e a propriedade aditiva do tensor das deformações, equação 2.1.2, o desenvolvimento da função de escoamento do modelo matemático originalmente proposto por Gurson, 1977, parte pela definição apresentada na equação 2.3.10, devendo agora o segundo invariante do tensor tensão, $J_{2}$, ser expresso em função do tensor das tensões relativas $(\boldsymbol{\eta})$, definido como:

$$
J_{2}=\frac{1}{2} \boldsymbol{\eta}: \boldsymbol{\eta} ; \boldsymbol{\eta}=\boldsymbol{S}-\boldsymbol{\beta}
$$

dessa forma a função de escoamento passa a ser reescrita conforme a equação 3.1.2.

$$
\phi=J_{2}(\boldsymbol{\eta})-\frac{1}{3}\left[1+f^{2}-2 f \cosh \left(\frac{3 p}{2 \sigma_{y}}\right)\right] \sigma_{y}^{2}
$$

A partir dessa equação, o primeiro passo para continuidade da elaboração do modelo matemático consiste na determinação do vetor de fluxo plástico, definido na equação 3.1.3.

$$
\boldsymbol{N}=\frac{\partial \phi}{\partial \boldsymbol{\sigma}}
$$


De forma que, aplicada a equação 3.1 .2 e executadas as devidas derivações e operações algébricas, o vetor de fluxo plástico será representado pela equação 3.1.5.

$$
\begin{gathered}
\boldsymbol{N}=\frac{\partial}{\partial \boldsymbol{\sigma}} J_{2}(\boldsymbol{\eta})-\frac{1}{3} \sigma_{y}^{2} \frac{\partial}{\partial \boldsymbol{\sigma}}\left[1+f^{2}-2 f \cosh \left(\frac{3 p}{2 \sigma_{y}}\right)\right] \\
\boldsymbol{N}=\boldsymbol{\eta}+\frac{1}{3} \sigma_{y} f \operatorname{senh}\left(\frac{3 p}{2 \sigma_{y}}\right) \boldsymbol{I},
\end{gathered}
$$

onde, decompondo em sua parcela desviadora e hidrostática, temos,

$$
\boldsymbol{N}=\boldsymbol{N}_{\boldsymbol{d}}+N_{v} \boldsymbol{I}
$$

$\log$,

$$
N_{d}=\eta
$$

e

$$
N_{v}=\frac{1}{3} \sigma_{y} f \operatorname{senh}\left(\frac{3 p}{2 \sigma_{y}}\right)
$$

A partir do resultado encontrado, determina-se a lei de fluxo plástico, apresentada na equação 3.1 .9 e que consiste no produto entre o vetor de fluxo plástico e um multiplicador plástico definido por $\gamma$.

$$
\begin{gathered}
\dot{\boldsymbol{\varepsilon}}^{p}=\dot{\gamma} \boldsymbol{N} \\
\dot{\boldsymbol{\varepsilon}}^{p}=\dot{\gamma}\left(\boldsymbol{N}_{\boldsymbol{d}}+N_{v} \boldsymbol{I}\right)
\end{gathered}
$$

Considerando a o passo distributivo realizado na equação 3.1.10 e a lei de fluxo plástico apresentada na equação 3.1.9., temos:

$$
\dot{\varepsilon}^{p}=\dot{\varepsilon}_{\boldsymbol{d}}^{p}+\frac{1}{3} \dot{\varepsilon}_{v}^{p} \boldsymbol{I}
$$

onde, a partir das definições postas nas equações 3.1.7. e 3.1.8., resulta-se: 


$$
\begin{aligned}
\dot{\boldsymbol{\varepsilon}}^{p} & =\dot{\gamma}\left[\boldsymbol{\eta}+\frac{1}{3} \sigma_{y} f \operatorname{senh}\left(\frac{3 p}{2 \sigma_{y}}\right) \boldsymbol{I}\right] \\
\dot{\boldsymbol{\varepsilon}}^{p} & =\dot{\gamma} \boldsymbol{\eta}+\frac{1}{3}\left[\dot{\gamma} \sigma_{y} f \operatorname{senh}\left(\frac{3 p}{2 \sigma_{y}}\right)\right] \boldsymbol{I}
\end{aligned}
$$

Além da lei de evolução do fluxo plástico, outras variáveis internas ainda precisam ter suas funções definidas como, por exemplo, a deformação plástica equivalente $\dot{\bar{\varepsilon}}^{p}$, o tensor das tensões cinemáticas $\boldsymbol{\beta}$ e a lei de evolução da fração volumétrica de vazios $f$. Sendo:

i. a deformação plástica equivalente " $\dot{\bar{\varepsilon}}^{p "}$;

$$
\dot{\bar{\varepsilon}}^{p}=\sqrt{\frac{2}{3} \dot{\varepsilon}^{p}: \dot{\varepsilon}^{p}}
$$

Substituindo a equação 3.1.13., operara-se matematicamente e expandese a expressão da plasticidade equivalente, equação 3.1.14, para:

$$
\dot{\bar{\varepsilon}}^{p}=\dot{\gamma} \sqrt{\frac{2}{3}\left\{\boldsymbol{\eta}: \boldsymbol{\eta}+\frac{1}{3}\left[\sigma_{y} f \operatorname{senh}\left(\frac{3 p}{2 \sigma_{y}}\right)\right]^{2}\right\}}
$$

ii. o tensor das tensões cinemáticas, $\boldsymbol{\beta}$;

$$
\dot{\boldsymbol{\beta}}=\frac{2}{3} H^{k} \dot{\boldsymbol{\varepsilon}}^{p}-b \dot{\bar{\varepsilon}}^{p} \boldsymbol{\beta}
$$

A partir do emprego da equação 3.1.7, 3.1.8 e 3.1.10, expande-se a função de evolução do tensor de encruamento cinemático para:

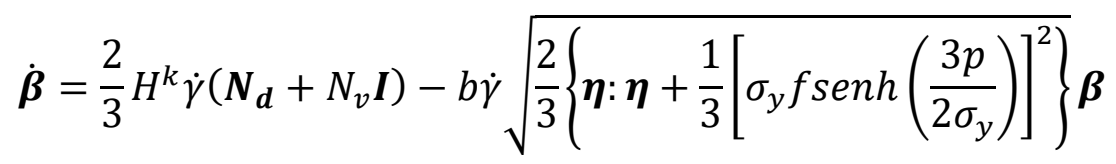


iii. a lei de evolução da fração volumétrica de vazios, $f$, anteriormente definida pela equação 2.3.9, onde, substituindo-se a parcela volumétrica do vetor de fluxo plástico, Eq. 3.1.8 e 3.1.9, temos:

$$
\dot{f}=(1-f) \dot{\gamma} \sigma_{y} f \operatorname{senh}\left(\frac{3 p}{2 \sigma_{y}}\right)
$$

Por fim, a condição de complementaridade de Kuhn-Tucker é fundamental para modelagem do comportamento elastoplástico e estabelece que a evolução da deformação pode ocorrer somente quando o estado de tensão estiver definido sobre a superfície de escoamento $\phi=0$, seguindo as condições expressas na equação 3.1.20.

$$
\dot{\gamma} \geq 0 ; \phi \leq 0 ; \dot{\gamma} \phi=0
$$


Quadro 1. Resumo do modelo matemático de Gurson com endurecimento cinemático de Armstrong e Frederick.

1. Decomposição aditiva da deformação;

$$
\varepsilon=\varepsilon^{e}+\varepsilon^{p}
$$

2. Lei Elástica;

$$
\sigma=\mathbb{D}^{e}: \varepsilon^{e}
$$

3. Função de escoamento;

$$
\phi=J_{2}(\boldsymbol{\eta})-\frac{1}{3} \sigma_{y}^{2}\left[1+f^{2}-2 f \cosh \left(\frac{3 p}{2 \sigma_{y}}\right)\right]
$$

onde,

$$
J_{2}=\frac{1}{2} \boldsymbol{\eta}: \boldsymbol{\eta} ; \boldsymbol{\eta}=\boldsymbol{S}-\boldsymbol{\beta}
$$

4. Lei de fluxo plástico;

$$
\dot{\varepsilon}^{p}=\dot{\gamma}\left(N_{d}+N_{v} I\right)
$$

onde,

$$
\begin{gathered}
\boldsymbol{N}_{\boldsymbol{d}}=\boldsymbol{\eta} \\
N_{v}=\frac{1}{3} \sigma_{y} f \operatorname{senh}\left(\frac{3 p}{2 \sigma_{y}}\right)
\end{gathered}
$$

Lei de outras variáveis internas:

$$
\begin{gathered}
\dot{\bar{\varepsilon}}^{p}=\dot{\gamma} \sqrt{\frac{2}{3}\left\{\boldsymbol{\eta}: \boldsymbol{\eta}+\frac{1}{3}\left[\sigma_{y} f \operatorname{senh}\left(\frac{3 p}{2 \sigma_{y}}\right)\right]^{2}\right\}} \\
\dot{\boldsymbol{\beta}}=\frac{2}{3} H^{k} \dot{\boldsymbol{\varepsilon}}^{p}-b \dot{\bar{\varepsilon}}^{p} \boldsymbol{\beta} \\
\dot{f}=(1-f) \dot{\gamma} \sigma_{y} f \operatorname{senh}\left(\frac{3 p}{2 \sigma_{y}}\right)
\end{gathered}
$$

5. Lei da complementaridade de Kuhn-Tucker:

$$
\dot{\gamma} \geq 0 ; \phi \leq 0 ; \dot{\gamma} \phi=0
$$




\subsection{DESENVOLVIMENTO DO MODELO NUMÉRICO}

Souza Neto, et al. (2008), introduz que materiais e modelos com comportamento dependente da trajetória, por exemplo, modelos elastoplásticos, têm sua solução em um conjunto de equações do modelo constitutivo e requer uma formulação através de um algoritmo de integração numérica (Souza Neto et al., 2008). Assim, para a solução do modelo matemático apresentado no tópico anterior se faz necessário formular um algoritmo capaz de atualizar as variáveis internas conhecidas.

Para tanto, deve-se estabelecer a concepção de um pseudo-intervalo de tempo $\left[t_{n}, t_{n+1}\right]$, onde o estado $n$ é conhecido e o estado $n+1$ é o qual se busca encontrar, sendo este uma tentativa dentro do algoritmo. A partir desse chamado "estado-tentativa", é aplicada uma discretização das equações constitutivas no pseudo-tempo, com base na metodologia de Euler implícita.

Já para atualizar as tensões, é utilizada a metodologia da decomposição do operador (Simo e Hughes, 1998; De Souza Neto et al. 2008), a qual se mostra em constante utilização em trabalhos de plasticidade computacional. Esse método baseia-se em dividir o problema em duas partes, o preditor elástico, aplicado para quando o problema ainda se encontra em uma fase completamente elástica e um corretor plástico, que compreende soluções através de um sistema de equações residuais formado pela lei elástica, pela função de escoamento e pelas equações de evolução, sendo este resolvido por meio do emprego de valores obtidos na construção do preditor elástico, o qual fornece os dados inicias do problema.

Nessa implementação, uma vez que a função de escoamento for violada, o corretor plástico será iniciado junto ao método de Newton-Rapshon, o qual foi utilizado para resolver o conjunto de equações não lineares discretizado.

Adiante serão determinados os algoritmos de retorno e o de Newton-Rapshon para resolução do sistema de equações não lineares do modelo Gurson com endurecimento cinemático segundo a lei de evolução da tensão de endurecimento cinemático de Armstrong e Frederick. $O$ algoritmo de integração numérica desenvolvido consta no Anexo II. 


\subsubsection{Algoritmo de retorno: Gurson com Armstrong-Frederick}

Estabelecido o conceito prévio a ser empregado neste capítulo, inicia-se o desenvolvimento do algoritmo de retorno do modelo de Gurson acoplado a ArmstrongFrederick, o qual utiliza como base as equações de atualização das seguintes variáveis:

i. do tensor de deformação elástico;

A partir da equação 2.1.1 e do conceito do mecanismo de evolução do tensor das deformações através da Figura 3, a equação 3.2.1 traz a expressão que representa a evolução da deformação elástica conforme a passagem do pseudo-tempo n+1.

$$
\varepsilon_{n+1}^{e}=\varepsilon_{n+1}^{e^{T}}-\Delta \varepsilon^{p}
$$

onde $\boldsymbol{\varepsilon}_{n+1}^{e^{T}}$ é o tensor deformação elástico tentativa no pseudo-tempo $n+1 \mathrm{e}$ $\Delta \varepsilon^{p}$ um incremento de deformação plástica que, durante o regime elástico, será nulo.

Aplicando a equação 3.1 .9 e subdividindo o vetor de fluxo plástico $\boldsymbol{N}$ em suas parcelas desviadora e volumétrica, Eq. 3.1.10, reescreve-se a 3.2.1 como sendo:

$$
\boldsymbol{\varepsilon}_{n+1}^{e}=\boldsymbol{\varepsilon}_{n+1}^{\boldsymbol{e}^{T}}-\Delta \gamma\left(\boldsymbol{N}_{\boldsymbol{d}_{n+1}}+N_{v_{n+1}} \boldsymbol{I}\right)
$$

onde substituindo os valores de $N_{\boldsymbol{d}_{n+1}}$ e $N_{v_{n+1}}$ em função das equações 3.1.7. e 3.1.9., tem-se:

$$
\boldsymbol{\varepsilon}_{n+1}^{e}=\boldsymbol{\varepsilon}_{n+1}^{\boldsymbol{e}^{T}}-\Delta \gamma\left[\boldsymbol{\eta}_{n+1}+\frac{1}{3} f_{n+1} \sigma_{y} \operatorname{senh}\left(\frac{3 p_{n+1}}{2 \sigma_{y}}\right) \boldsymbol{I}\right]
$$

ii. as componentes do tensor tensão;

Considerando a lei de Hooke generalizada expressa pela equação 2.1.1 e que a deformação elástica no tempo $n+1$ será a deformação elástica tentativa subtraída a parcela plástica segundo a lei de fluxo plástico, tem-se, conforme Eq. 3.2.5 que :

$$
\boldsymbol{\sigma}_{n+1}=\mathbb{D}^{e}: \boldsymbol{\varepsilon}_{n+1}^{\boldsymbol{e}^{T}}-\Delta \gamma \mathbb{D}^{e}: \boldsymbol{N}_{n+1}
$$


e, retornando esta representação para expressão em função do tensor tensão de Cauchy, com aplicação dos módulos volumétricos $(K)$ e de cisalhamento $(G)$ do material, apresenta-se a equação 3.2.5 como:

$$
\boldsymbol{\sigma}_{n+1}=\boldsymbol{\sigma}_{n+1}^{T}-2 G \Delta \gamma \boldsymbol{N}_{\boldsymbol{d}_{n+1}}-K \Delta \gamma N_{v_{n+1}} \boldsymbol{I}
$$

onde, substituindo as expressões apresentados nas equações 3.1.7. e 3.1.8., tem-se:

$$
\boldsymbol{\sigma}_{n+1}=\boldsymbol{\sigma}_{n+1}^{T}-2 G \Delta \gamma \boldsymbol{\eta}_{n+1}-\frac{1}{3} K \Delta \gamma f_{n+1} \sigma_{y} \operatorname{senh}\left(\frac{3 p_{n+1}}{2 \sigma_{y}}\right) \boldsymbol{I},
$$

logo, decompondo o tensor tensão Cauchy em sua parcela desviadora e hidrostática, resulta-se na expressão dada pela Eq 3.2.8,

$$
\boldsymbol{S}_{n+1}+p_{n+1} \boldsymbol{I}=\boldsymbol{S}_{n+1}^{T}+p_{n+1}^{T} \boldsymbol{I}-2 G \Delta \gamma \boldsymbol{N}_{\boldsymbol{d}_{n+1}}-K \Delta \gamma N_{v_{n+1}} \boldsymbol{I},
$$

onde organizando os termos algebricamente, conforme sua natureza, permitese destacar as seguintes equação:

$$
\begin{gathered}
\boldsymbol{S}_{n+1}=\boldsymbol{S}_{n+1}^{T}-2 G \Delta \gamma \boldsymbol{N}_{\boldsymbol{d}_{n+1}} \\
p_{n+1}=p_{n+1}^{T}-K \Delta \gamma N_{v_{n+1}}
\end{gathered}
$$

iii. a deformação equivalente;

Assim como para o tensor da deformação plástica, a deformação equivalente terá seu módulo no tempo $n+1$ como sendo a deformação equivalente tentativa subtraída sua parcela plástica, ou seja,

$$
\bar{\varepsilon}_{n+1}^{p}=\bar{\varepsilon}_{n}^{p}+\Delta \bar{\varepsilon}^{p},
$$

onde, substituindo a equação 3.1.15. na equação acima, encontra-se:

$$
\bar{\varepsilon}_{n+1}^{p}=\bar{\varepsilon}_{n}^{p}+\Delta \gamma \sqrt{\frac{2}{3}\left\{\boldsymbol{\eta}_{n+1}: \boldsymbol{\eta}_{n+1}+\frac{1}{3}\left[\sigma_{y} f_{n+1} \operatorname{senh}\left(\frac{3 p_{n+1}}{2 \sigma_{y}}\right)\right]^{2}\right\}},
$$


Em continuidade as expressões já determinadas para o algoritmo em questão, dá-se agora sequência da atualização das variáveis internas, seguindo o mesmo princípio do somatório de um incremento da variável em questão a um passo tentativa do pseudotempo definido, dentro dos critérios do modelo de Gurson, como sendo:

iv. atualização da função volumétrica de vazios;

$$
f_{n+1}=f_{n+1}^{T}+\Delta f
$$

onde a substituindo das equações 2.3.9., 3.1.8 e 3.1.9 na equação acima resulta na forma:

$$
f_{n+1}=f_{n+1}^{T}+\left(1-f_{n+1}\right) \Delta \gamma f_{n+1} \sigma_{y} \operatorname{senh}\left(\frac{3 p_{n+1}}{2 \sigma_{y}}\right)
$$

v. atualização do tensor das tensões cinemáticas;

$$
\boldsymbol{\beta}_{n+1}=\boldsymbol{\beta}_{n+1}^{T}+\Delta \boldsymbol{\beta}
$$

de forma que, a partir do emprego das equações 3.1.16, 3.1.7, 3.1.8 e 3.1.10, expande-se a função de evolução do tensor de encruamento cinemático para:

$$
\begin{aligned}
\boldsymbol{\beta}_{n+1}=\boldsymbol{\beta}_{n+1}^{T} & +\frac{2}{3} H^{k} \Delta \gamma\left(\boldsymbol{N}_{\boldsymbol{d}_{n+1}}+N_{v_{n+1}} \boldsymbol{I}\right) \\
& -b \Delta \gamma \sqrt{\frac{2}{3}\left\{\boldsymbol{\eta}_{n+1}: \boldsymbol{\eta}_{n+1}+\frac{1}{3}\left[\sigma_{y} f_{n+1} \operatorname{senh}\left(\frac{3 p_{n+1}}{2 \sigma_{y}}\right)\right]^{2}\right\}} \boldsymbol{\beta}_{n+1}
\end{aligned}
$$

Por fim, definidas as funções de atualização, estabelece-se a atualização da função de escoamento de Gurson dentro dos parâmetros do estado tentativa, resultando em:

$$
\phi_{n+1}=J_{2}(\boldsymbol{\eta})_{n+1}-\frac{1}{3} \sigma_{y}^{2}\left[1+f_{n+1}^{2}-2 f_{n+1} \cosh \left(\frac{3 p_{n+1}}{2 \sigma_{y}}\right)\right]
$$


1. Determinação de um estado tentativa e um incremento $\Delta \varepsilon$;

$$
\begin{gathered}
\boldsymbol{\varepsilon}_{n+1}^{\boldsymbol{e}^{T}}=\boldsymbol{\varepsilon}_{n}^{\boldsymbol{e}}+\Delta \boldsymbol{\varepsilon} \quad ; \quad f_{n+1}=f_{n} \quad ; \quad \boldsymbol{\beta}_{n+1}=\boldsymbol{\beta}_{n} \\
\boldsymbol{\sigma}_{n+1}^{\boldsymbol{T}}=\mathbb{D}^{\boldsymbol{e}}: \boldsymbol{\varepsilon}_{n+1}^{\boldsymbol{e}^{T}} \quad ; \quad \bar{\varepsilon}_{n+1}^{p}=\bar{\varepsilon}_{n}^{p}
\end{gathered}
$$

2. Admissibilidade;

$$
\phi_{n+1}^{T}=J_{2}^{T}(\boldsymbol{\eta})_{n+1}-\frac{1}{3} \sigma_{y}^{2}\left[1+f_{n+1}^{T^{2}}-2 f_{n+1}^{T} \cosh \left(\frac{3 p_{n+1}^{T}}{2 \sigma_{y}}\right)\right]
$$

se, $\phi_{n+1}^{T} \leq 0$

passo elástico: $(*)_{n+1}=(*)_{n+1}^{T}$;

caso contrário, passo plástico: aplicar corretor plástico;

3. Corretor plástico;

Resolver o sistema de equações não lineares, tendo como variáveis: $\boldsymbol{S}_{n+1}, p_{n+1}, f_{n+1}, \Delta \gamma$ e $\boldsymbol{\beta}_{n+1}$

(provenientes das equações: 3.2.9.; 3.2.10.; 3.2.17.; 3.2.14.; 3.2.16.)

$$
\begin{aligned}
& \boldsymbol{S}_{n+1}=\boldsymbol{S}_{n+1}^{T}-2 G \Delta \gamma \boldsymbol{N}_{\boldsymbol{d}_{n+1}} \\
& p_{n+1}=p_{n+1}^{T}-K \Delta \gamma N_{v_{n+1}} \\
& f_{n+1}=f_{n+1}^{T}+\left(1-f_{n+1}\right) \Delta \gamma f_{n+1} \sigma_{y} \operatorname{senh}\left(\frac{3 p_{n+1}}{2 \sigma_{y}}\right) \\
& \phi_{n+1}=J_{2}(\boldsymbol{\eta})_{n+1}-\frac{1}{3}\left[1+f_{n+1}^{2}-2 f_{n+1} \cosh \left(\frac{3 p_{n+1}}{2 \sigma_{y}}\right)\right] \sigma_{y}^{2} \\
& \boldsymbol{\beta}_{n+1}=\boldsymbol{\beta}_{n+1}^{T}+\frac{2}{3} H^{k} \Delta \gamma\left(\boldsymbol{N}_{\boldsymbol{d}_{n+1}}+N_{v_{n+1}} \boldsymbol{I}\right)-b \bar{\varepsilon}_{n+1}^{p} \boldsymbol{\beta}_{n+1}
\end{aligned}
$$

atualizar outras variáveis;

$$
\begin{gathered}
\boldsymbol{\varepsilon}_{n+1}^{\boldsymbol{e}}=\boldsymbol{\varepsilon}_{n+1}^{\boldsymbol{e}^{T}}-\Delta \gamma \boldsymbol{N}_{n+1} \\
\bar{\varepsilon}_{n+1}^{p}=\bar{\varepsilon}_{n}^{p}+\Delta \gamma \sqrt{\frac{2}{3}\left\{\boldsymbol{\eta}_{n+1}: \boldsymbol{\eta}_{n+1}+\frac{1}{3}\left[\sigma_{y} f_{n+1} \operatorname{senh}\left(\frac{3 p_{n+1}}{2 \sigma_{y}}\right)\right]^{2}\right\}}
\end{gathered}
$$

4. Fim. 


\subsubsection{Algoritmo de Newton-Raphson: Sistema de equações não linear}

Para que se estabeleça o corretor plástico, primeiramente deve-se considerar valores iniciais aleatórios para as variáveis como sendo:

$$
\begin{gathered}
\boldsymbol{S}_{n+1}^{(0)}=\boldsymbol{S}_{n+1}^{T} ; \quad p_{n+1}^{(0)}=p_{n+1}^{T} ; \Delta \gamma^{(0)}=0 \\
f_{n+1}^{(0)}=f_{n} ; \boldsymbol{\beta}_{n+1}^{(0)}=\boldsymbol{\beta}_{n},
\end{gathered}
$$

em que $n+1$ representa o pseudo-tempo do estado tentativa.

Sequencialmente, reescreve-se o sistema de equações não lineares na forma de equações residuais, resultando nas expressões:

$$
\begin{aligned}
& R_{\boldsymbol{S}_{n+1}}=\boldsymbol{S}_{n+1}-\boldsymbol{S}_{n+1}^{T}+2 G \Delta \gamma \boldsymbol{N}_{\boldsymbol{d}_{n+1}} \\
& R_{p_{n+1}}=p_{n+1}-p_{n+1}^{T}+K \Delta \gamma N_{v_{n+1}} \\
& R_{f_{n+1}}=f_{n+1}-f_{n+1}^{T}-\left(1-f_{n+1}\right) \Delta \gamma f_{n+1} \sigma_{y} \operatorname{senh}\left(\frac{3 p_{n+1}}{2 \sigma_{y}}\right) \\
& R_{\Delta \gamma}=\frac{1}{2} \boldsymbol{\eta}_{n+1}: \boldsymbol{\eta}_{n+1}-\frac{1}{3} \sigma_{y}^{2}\left[1+f_{n+1}^{2}-2 f_{n+1} \cosh \left(\frac{3 p_{n+1}}{2 \sigma_{y}}\right)\right] \\
& R_{\boldsymbol{\beta}_{n+1}=} \boldsymbol{\beta}_{n+1}-\boldsymbol{\beta}_{n+1}^{T}-\frac{2}{3} H^{k} \Delta \gamma\left(\boldsymbol{N}_{\boldsymbol{d}_{n+1}}+N_{v_{n+1}} \boldsymbol{I}\right) \\
& \quad+b \Delta \gamma \sqrt{\frac{2}{3}\left\{\boldsymbol{\eta}_{n+1}: \boldsymbol{\eta}_{n+1}+\frac{1}{3}\left[\sigma_{y} f_{n+1} \operatorname{senh}\left(\frac{3 p_{n+1}}{2 \sigma_{y}}\right)\right]^{2}\right\}} \boldsymbol{\beta}_{n+1}
\end{aligned}
$$

Desta forma, as equações residuais descritas podem ser linearizadas na forma do sistema:

$$
\left[\frac{\partial R_{(*)}}{\partial(*)}\right]^{k} \cdot[\delta(*)]^{k+1}=-\left[R_{(*)}\right]^{k},
$$

logo, o sistema matricial a ser solucionado será: 


$$
\left[\begin{array}{lllll}
\frac{\partial R_{\boldsymbol{S}_{n+1}}}{\partial \boldsymbol{S}_{n+1}} & \frac{\partial R_{\boldsymbol{S}_{n+1}}}{\partial p_{n+1}} & \frac{\partial R_{\boldsymbol{S}_{n+1}}}{\partial f_{n+1}} & \frac{\partial R_{\boldsymbol{S}_{n+1}}}{\partial \Delta \gamma} & \frac{\partial R_{\boldsymbol{S}_{n+1}}}{\partial \boldsymbol{\beta}_{n+1}} \\
\frac{\partial R_{p_{n+1}}}{\partial \boldsymbol{S}_{n+1}} & \frac{\partial R_{p_{n+1}}}{\partial p_{n+1}} & \frac{\partial R_{p_{n+1}}}{\partial f_{n+1}} & \frac{\partial R_{p_{n+1}}}{\partial \Delta \gamma} & \frac{\partial R_{p_{n+1}}}{\partial \boldsymbol{\beta}_{n+1}} \\
\frac{\partial R_{\Delta \gamma}}{\partial \boldsymbol{S}_{n+1}} & \frac{\partial R_{\Delta \gamma}}{\partial p_{n+1}} & \frac{\partial R_{\Delta \gamma}}{\partial f_{n+1}} & \frac{\partial R_{\Delta \gamma}}{\partial \Delta \gamma} & \frac{\partial R_{\Delta \gamma}}{\partial \boldsymbol{\beta}_{n+1}} \\
\frac{\partial R_{f_{n+1}}}{\partial \boldsymbol{S}_{n+1}} & \frac{\partial R_{f_{n+1}}}{\partial p_{n+1}} & \frac{\partial R_{f_{n+1}}}{\partial f_{n+1}} & \frac{\partial R_{f_{n+1}}}{\partial \Delta \gamma} & \frac{\partial R_{f_{n+1}}}{\partial \boldsymbol{\beta}_{n+1}} \\
\frac{\partial R_{\boldsymbol{\beta}_{n+1}}}{\partial \boldsymbol{S}_{n+1}} & \frac{\partial R_{\boldsymbol{\beta}_{n+1}}}{\partial p_{n+1}} & \frac{\partial R_{\boldsymbol{\beta}_{n+1}}}{\partial f_{n+1}} & \frac{\partial R_{\boldsymbol{\beta}_{n+1}}}{\partial \Delta \gamma} & \frac{\partial R_{\boldsymbol{\beta}_{n+1}}}{\partial \boldsymbol{\beta}_{n+1}}
\end{array}\right]^{k+1} \cdot\left[\begin{array}{c}
\delta \boldsymbol{S}_{n+1} \\
\delta p_{n+1} \\
\delta f_{n+1} \\
\delta \Delta \gamma \\
\delta \boldsymbol{\beta}_{n+1}
\end{array}\right]^{R_{\boldsymbol{S}_{n+1}}}{ }^{k}=-\left[\begin{array}{c}
R_{p_{n+1}} \\
R_{f_{n+1}} \\
R_{\Delta \gamma} \\
R_{\boldsymbol{\beta}_{n+1}}
\end{array}\right]^{2}
$$

Eq. 3.2.24

Antes da solução do sistema, determina-se que o erro de convergência estará ligado a tolerância desejada, conforme segue e considerando que este trabalho empregou uma tolerância equivalente a $10^{-6}$ para implementação do modelo numérico.

$$
\text { Erro }=\frac{\phi_{n+1}}{\sigma y} \leq \text { tolerância }
$$

Por fim, solucionam-se as derivadas do sistema de equações residuais resultando em:

i. primeira equação residual;

$$
\frac{\partial R_{\boldsymbol{S}_{n+1}}}{\partial \boldsymbol{S}_{n+1}}=\boldsymbol{I}^{4}+2 G \Delta \gamma \frac{\partial \boldsymbol{N}_{\boldsymbol{d}_{n+1}}}{\partial \boldsymbol{S}_{n+1}}
$$

A solução da derivada $\frac{\partial N_{d_{n+1}}}{\partial S_{n+1}}$ consta no item i do Anexo I.

$$
\begin{aligned}
& \frac{\partial R_{\boldsymbol{S}_{n+1}}}{\partial p_{n+1}}=0 ; \\
& \frac{\partial R_{\boldsymbol{S}_{n+1}}}{\partial f_{n+1}}=0 ; \\
& \frac{\partial R_{\boldsymbol{S}_{n+1}}}{\partial \Delta \gamma}=2 G \boldsymbol{N}_{\boldsymbol{d}_{n+1}} ;
\end{aligned}
$$


$\frac{\partial R_{\boldsymbol{S}_{n+1}}}{\partial \boldsymbol{\beta}_{n+1}}=2 G \Delta \gamma \frac{\partial \boldsymbol{N}_{\boldsymbol{d}_{n+1}}}{\partial \boldsymbol{\beta}_{n+1}}$

Eq. 3.2 .30

A solução da derivada $\frac{\partial N_{d_{n+1}}}{\partial \boldsymbol{\beta}_{n+1}}$ consta no item ii do Anexo I.

ii. segunda equação residual

$$
\begin{aligned}
& \frac{\partial R_{p_{n+1}}}{\partial \boldsymbol{S}_{n+1}}=0 \\
& \frac{\partial R_{p_{n+1}}}{\partial p_{n+1}}=1+K \Delta \gamma \frac{\partial N_{v_{n+1}}}{\partial p_{n+1}} ;
\end{aligned}
$$

Eq. 3.2.32

A solução da derivada $\frac{\partial N_{v_{n+1}}}{\partial p_{n+1}}$ consta no item iii do Anexo I.

$$
\frac{\partial R_{p_{n+1}}}{\partial f_{n+1}}=K \Delta \gamma \frac{\partial N_{v_{n+1}}}{\partial f_{n+1}}
$$

A solução da derivada $\frac{\partial N_{v_{n+1}}}{\partial f_{n+1}}$ consta no item iv do Anexo I.

$$
\begin{aligned}
& \frac{\partial R_{p_{n+1}}}{\partial \Delta \gamma}=K N_{v_{n+1}} \\
& \frac{\partial R_{p_{n+1}}}{\partial \boldsymbol{\beta}_{n+1}}=0 ;
\end{aligned}
$$

Eq. 3.2.35

iii. terceira equação residual;

$$
\frac{\partial R_{f_{n+1}}}{\partial \boldsymbol{S}_{n+1}}=0 ;
$$




$$
\begin{aligned}
& \frac{\partial R_{f_{n+1}}}{\partial p_{n+1}}=-\left(1-f_{n+1}\right) \frac{3}{2} \Delta \gamma f_{n+1} \cosh \left(\frac{3 p_{n+1}}{2 \sigma_{y}}\right) \\
& \frac{\partial R_{f_{n+1}}}{\partial f_{n+1}}=1-\left[\left(1-2 f_{n+1}\right) \Delta \gamma \sigma_{y} \operatorname{senh}\left(\frac{3 p_{n+1}}{2 \sigma_{y}}\right)\right] \\
& \frac{\partial R_{f_{n+1}}}{\partial \Delta \gamma}=-\left(1-2 f_{n+1}\right) f_{n+1} \sigma_{y} \operatorname{senh}\left(\frac{3 p_{n+1}}{2 \sigma_{y}}\right) ; \\
& \frac{\partial R_{f_{n+1}}}{\partial \boldsymbol{\beta}_{n+1}}=0 ;
\end{aligned}
$$

iv. quarta equação residual;

$$
\begin{aligned}
& \frac{\partial R_{\Delta \gamma}}{\partial \boldsymbol{S}_{n+1}}=\boldsymbol{\eta}_{n+1} \\
& \frac{\partial R_{\Delta \gamma}}{\partial p_{n+1}}=2 f_{n+1} \sigma_{y} \operatorname{senh}\left(\frac{3 p_{n+1}}{2 \sigma_{y}}\right) ; \\
& \frac{\partial R_{\Delta \gamma}}{\partial f_{n+1}}=-\frac{1}{3} \sigma_{y}\left[2 f_{n+1}-2 \cosh \left(\frac{3 p_{n+1}}{2 \sigma_{y}}\right)\right] \\
& \frac{\partial R_{\Delta \gamma}}{\partial \Delta \gamma}=0 \\
& \frac{\partial R_{\Delta \gamma}}{\partial \boldsymbol{\beta}_{n+1}}=-\boldsymbol{\eta}_{n+1}
\end{aligned}
$$

v. quinta equação residual;

Assumindo $A=\frac{2}{3}\left\{\boldsymbol{\eta}_{n+1}: \boldsymbol{\eta}_{n+1}+\frac{1}{3}\left[\sigma_{y} f_{n+1} \operatorname{senh}\left(\frac{3 p_{n+1}}{2 \sigma_{y}}\right)\right]^{2}\right\}$, tem-se: 


$$
\frac{\partial R_{\boldsymbol{\beta}_{n+1}}}{\partial \boldsymbol{S}_{n+1}}=-\frac{2}{3} H^{k} \Delta \gamma\left[\frac{\partial \boldsymbol{N}_{\boldsymbol{d}_{n+1}}}{\partial \boldsymbol{S}_{n+1}}\right]+b \Delta \gamma \frac{\partial \sqrt{A}}{\partial \boldsymbol{S}_{n+1}} \otimes \boldsymbol{\beta}_{n+1}
$$

A solução da derivada $\frac{\partial \boldsymbol{N}_{d_{n+1}}}{\partial \boldsymbol{S}_{n+1}}$ e da expressão $\frac{\partial \sqrt{A}}{\partial \boldsymbol{s}_{n+1}} \otimes \boldsymbol{\beta}_{n+1}$ constam, respectivamente, nos itens i e vii do Anexo I.

$$
\frac{\partial R_{\boldsymbol{\beta}_{n+1}}}{\partial p_{n+1}}=-\frac{2}{3} H^{k} \Delta \gamma\left[\frac{\partial N_{v_{n+1}}}{\partial p_{n+1}} \boldsymbol{I}\right]+b \Delta \gamma \frac{\partial \sqrt{A}}{\partial p_{n+1}} \boldsymbol{\beta}_{n+1}
$$

A solução das derivadas $\frac{\partial N_{v_{n+1}}}{\partial p_{n+1}}$ e $\frac{\partial \sqrt{A}}{\partial p_{n+1}}$ constam, respectivamente, nos itens iii e viii do Anexo I.

$$
\frac{\partial R_{\boldsymbol{\beta}_{n+1}}}{\partial f_{n+1}}=-\frac{2}{3} H^{k} \Delta \gamma\left[\frac{\partial N_{v_{n+1}}}{\partial f_{n+1}} \boldsymbol{I}\right]+b \Delta \gamma \frac{\partial \sqrt{A}}{\partial f_{n+1}} \boldsymbol{\beta}_{n+1} ;
$$

A solução das derivadas $\frac{\partial N_{v_{n+1}}}{\partial f_{n+1}}$ e $\frac{\partial \sqrt{A}}{\partial f_{n+1}}$ constam, respectivamente, nos itens iv e ix do Anexo I.

$$
\begin{aligned}
& \frac{\partial R_{\boldsymbol{\beta}_{n+1}}}{\partial \Delta \gamma}=-\frac{2}{3} H^{k}\left(\boldsymbol{N}_{\boldsymbol{d}_{n+1}}+N_{v_{n+1}} \boldsymbol{I}\right)+b \sqrt{A} \boldsymbol{\beta}_{n+1} \\
& \frac{\partial R_{\boldsymbol{\beta}_{n+1}}}{\partial \boldsymbol{\beta}_{n+1}}=\boldsymbol{I}^{4}-\frac{2}{3} H^{k} \Delta \gamma\left[\frac{\partial \boldsymbol{N}_{\boldsymbol{d}_{n+1}}}{\partial \boldsymbol{\beta}_{n+1}}\right]+b \Delta \gamma\left(\frac{\partial \sqrt{A}}{\partial \boldsymbol{\beta}_{n+1}} \otimes \boldsymbol{\beta}_{n+1}+\sqrt{A} \boldsymbol{I}^{4}\right)
\end{aligned}
$$

A solução da derivada $\frac{\partial \boldsymbol{N}_{d_{n+1}}}{\partial \boldsymbol{\beta}_{n+1}}$ e da expressão $\frac{\partial \sqrt{A}}{\partial \boldsymbol{\beta}_{n+1}} \otimes \boldsymbol{\beta}_{n+1}$ constam, respectivamente, nos itens ii e x do Anexo I. 
Quadro 3. Algoritmo de resolução do sistema não linear para o modelo de Gurson com endurecimento cinemático de Armstrong e Frederick.

1. Dados o estado tentativa como parâmetros iniciais;

$$
\begin{gathered}
\boldsymbol{S}_{n+1}^{(0)}=\boldsymbol{S}_{n+1}^{T} ; \quad p_{n+1}^{(0)}=p_{n+1}^{T} ; \Delta \gamma^{(0)}=0 \\
f_{n+1}^{(0)}=f_{n} ; \boldsymbol{\beta}_{n+1}^{(0)}=\boldsymbol{\beta}_{n}
\end{gathered}
$$

2. Resolver o Sistema de equações para $\boldsymbol{S}_{n+1}, p_{n+1}, f_{n+1}, \Delta \gamma$ e $\boldsymbol{\beta}_{n+1}$;

$$
\left.\left[\begin{array}{lllll}
\frac{\partial R_{\boldsymbol{S}_{n+1}}}{\partial \boldsymbol{S}_{n+1}} & \frac{\partial R_{\boldsymbol{S}_{n+1}}}{\partial p_{n+1}} & \frac{\partial R_{\boldsymbol{S}_{n+1}}}{\partial f_{n+1}} & \frac{\partial R_{\boldsymbol{S}_{n+1}}}{\partial \Delta \gamma} & \frac{\partial R_{\boldsymbol{S}_{n+1}}}{\partial \boldsymbol{\beta}_{n+1}} \\
\frac{\partial R_{p_{n+1}}}{\partial \boldsymbol{S}_{n+1}} & \frac{\partial R_{p_{n+1}}}{\partial p_{n+1}} & \frac{\partial R_{p_{n+1}}}{\partial f_{n+1}} & \frac{\partial R_{p_{n+1}}}{\partial \Delta \gamma} & \frac{\partial R_{p_{n+1}}}{\partial \boldsymbol{\beta}_{n+1}} \\
\frac{\partial R_{\Delta \gamma}}{\partial \boldsymbol{S}_{n+1}} & \frac{\partial R_{\Delta \gamma}}{\partial p_{n+1}} & \frac{\partial R_{\Delta \gamma}}{\partial f_{n+1}} & \frac{\partial R_{\Delta \gamma}}{\partial \Delta \gamma} & \frac{\partial R_{\Delta \gamma}}{\partial \boldsymbol{\beta}_{n+1}} \\
\frac{\partial R_{f_{n+1}}}{\partial \boldsymbol{S}_{n+1}} & \frac{\partial R_{f_{n+1}}}{\partial p_{n+1}} & \frac{\partial R_{f_{n+1}}}{\partial f_{n+1}} & \frac{\partial R_{f_{n+1}}}{\partial \Delta \gamma} & \frac{\partial R_{f_{n+1}}}{\partial \boldsymbol{\beta}_{n+1}} \\
\frac{\partial R_{\boldsymbol{\beta}_{n+1}}}{\partial \boldsymbol{S}_{n+1}} & \frac{\partial R_{\boldsymbol{\beta}_{n+1}}}{\partial p_{n+1}} & \frac{\partial R_{\boldsymbol{\beta}_{n+1}}}{\partial f_{n+1}} & \frac{\partial R_{\boldsymbol{\beta}_{n+1}}}{\partial \Delta \gamma} & \frac{\partial R_{\boldsymbol{\beta}_{n+1}}}{\partial \boldsymbol{\beta}_{n+1}}
\end{array}\right]^{k+1} \cdot\left[\begin{array}{c}
\delta \boldsymbol{S}_{n+1} \\
\delta p_{n+1} \\
\delta f_{n+1} \\
\delta \Delta \gamma \\
\delta \boldsymbol{\beta}_{n+1}
\end{array}\right]^{R_{\boldsymbol{S}_{n+1}}}\right]^{k}=-\left[\begin{array}{c}
R_{p_{n+1}} \\
R_{f_{n+1}} \\
R_{\Delta \gamma} \\
R_{\boldsymbol{\beta}_{n+1}}
\end{array}\right]^{2}
$$

3. Calcular;

$$
\begin{gathered}
\boldsymbol{S}_{n+1}^{(k+1)}=\boldsymbol{S}_{n+1}^{(k)}+\delta \boldsymbol{S}_{n+1}^{(k+1)} \quad ; \quad p_{n+1}^{(k+1)}=p_{n+1}^{(k)}+\delta p_{n+1}^{(k+1)} \\
\Delta \gamma^{(k+1)}=\Delta \gamma^{(k)}=\delta \Delta \gamma^{(k+1)} ; \\
\boldsymbol{\beta}_{n+1}^{(k+1)}=\boldsymbol{\beta}_{n+1}^{(k)}+\delta \boldsymbol{\beta}_{n+1}^{(k+1)}
\end{gathered}
$$

4. Verificar a convergência.

$$
\text { Erro }=\frac{\phi_{n+1}}{\sigma y} \leq \text { tolerância }
$$




\section{RESULTADOS E DISCUSSÕES}

Neste capítulo serão apresentados os resultados da implementação do modelo de Gurson com endurecimento cinemático segundo a lei de Armstrong-Frederick, utilizado para previsão de vida à fadiga com base na micromecânica de defeitos.

Para tanto, os algoritmos apresentados no Capítulo 03 e resumidos nos Quadros 02 e 03, tiveram sua implementação realizada através de uma ferramenta acadêmica de Elementos Finitos, onde se utilizou a linguagem FORTRAN. O resultado desse trabalho numérico consta no Anexo II.

O modelo desenvolvido foi aplicado para análise de três materiais, sendo: os aços 304 e S460N e o alumínio 6061-T6. Todos submetidos a carregamentos uniaxiais e multiaxiais proporcionais e não proporcionais.

\subsection{PARÂMETROS DO MATERIAL}

Para se utilizar o modelo de Gurson com endurecimento cinemático de Armstrong-Frederick se fez necessária a obtenção de parâmetros materiais, sendo: o coeficiente de endurecimento cinemático $H^{k}$, um parâmetro material $b$ e a fração volumétrica de vazios inicial $f_{o}$.

Os parâmetros associados com a evolução das tensões cinemáticas e com o parâmetro de saturação de $\boldsymbol{\beta}$ serão adotados com base na relação de Ramberg-Osgood (1943), equação 4.1.1.

$$
\varepsilon_{a}^{p}=\left(\frac{\sigma_{a}}{H^{\prime}}\right)^{\frac{1}{n^{\prime}}}
$$

onde $\sigma_{a}$ e $\varepsilon_{a}^{p}$ são as amplitudes de tensão e deformação, respectivamente.

$\mathrm{Na}$ Equação 4.1.1, o coeficiente de endurecimento $H^{\prime}$ e $n^{\prime}$ são parâmetros que descrevem o encruamento do material, os quais foram obtidos de Jiang et al. (2007) para o aço S460N e Itoh (2001) para o aço 304 e para o alumínio 6061-T6. 
Seguindo os dados experimentais e a rotina Matlab® empregada por Lopes (2016), a Tabela 01 apresenta os resultados da calibração atingida através do ajuste dos parâmetros $H^{k}$, b e $\sigma_{y_{0}}$ da integração da equação analítica de Armstrong-Frederick, Eq. 2.2.4, em relação à curva de Ramberg-Osgood, equação 4.1.1.

Tabela 1. Resultados da calibração por amplitude para o modelo de Armstrong-Frederick e parâmetros da curva de Ramberg-Osgood. Fonte: adaptado, Pereira, 2014.

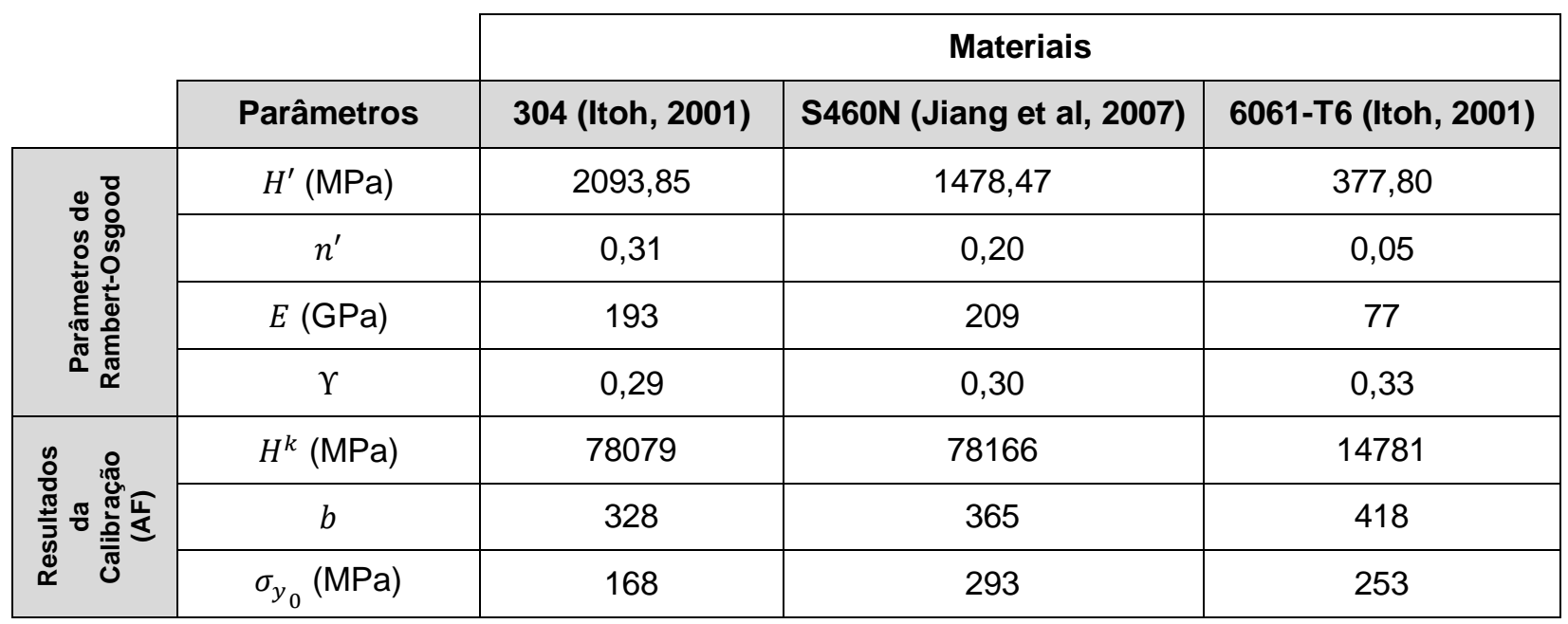

Observa-se que parâmetro da porosidade inicial $f_{o}$ será associado a evolução do dano ou da fração volumétrica de vazios e, segundo Gurson (1977), este será calibrado a partir de uma trajetória axial, Figura 15. Nessa linha, através da aplicação de uma amplitude de deformação e de um número de ciclos para uma falha conhecida, foi possível se estabelecer os dados das Tabela 2, Tabela 3 e Tabela 4, os quais apresentam a vida experimental de cada material citado. (Itoh, 2001) (Jiang et al, 2007)

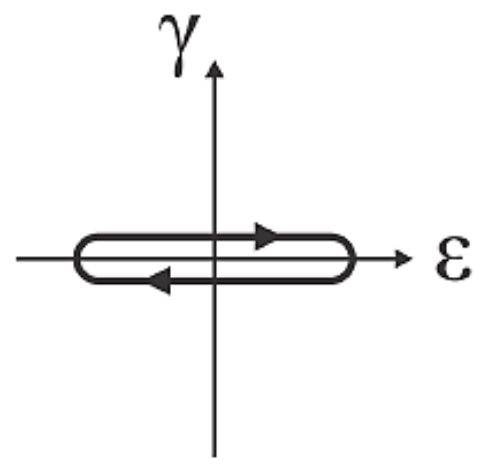

Figura 15. Trajetória uniaxial de tração-compressão imposta para calibração. Fonte: Jiang et al, 2007. 
Tabela 2. Resultados de identificação de vida experimental para o aço 304. Fonte: Itoh (2001).

\begin{tabular}{|c|c|c|}
\hline \multicolumn{3}{|c|}{304 (Itoh, 2001) } \\
\hline $\boldsymbol{\varepsilon}_{\boldsymbol{a}}$ (\%) & $\boldsymbol{N}_{\text {exp }}$ (Ciclos) & $\boldsymbol{f}_{\boldsymbol{o}}$ \\
\hline 0,600 & 690 & $5,60 \cdot 10^{-5}$ \\
\hline 0,500 & 1500 & $5,50 \cdot 10^{-5}$ \\
\hline 0,400 & 7100 & $2,47 \cdot 10^{-5}$ \\
\hline 0,325 & 23400 & $1,40 \cdot 10^{-5}$ \\
\hline 0,250 & 49000 & $1,32 \cdot 10^{-5}$ \\
\hline
\end{tabular}

Tabela 3. Resultados de identificação de vida experimental para o aço S460N. Fonte: Jiang, et al. (2007).

\begin{tabular}{|c|c|c|}
\hline \multicolumn{3}{|c|}{ S460N (Jiang et al, 2007) } \\
\hline $\boldsymbol{\varepsilon}_{\boldsymbol{a}}$ (\%) & $\boldsymbol{N}_{\text {exp }}$ (Ciclos) & $\boldsymbol{f}_{\boldsymbol{o}}$ \\
\hline 0,500 & 1600 & $2,37 \cdot 10^{-4}$ \\
\hline 0,330 & 7690 & $1,47 \cdot 10^{-4}$ \\
\hline 0,220 & 33100 & $1,08 \cdot 10^{-4}$ \\
\hline
\end{tabular}

Tabela 4. Resultados de identificação de vida experimental para o alumínio 6061-T6. Fonte: Itoh (2001).

\begin{tabular}{|c|c|c|}
\hline \multicolumn{3}{|c|}{ 6061-T6 (Itoh, 2001) } \\
\hline $\boldsymbol{\varepsilon}_{\boldsymbol{a}}$ (\%) & $\boldsymbol{N}_{\text {exp }}$ (Ciclos) & $\boldsymbol{f}_{\boldsymbol{o}}$ \\
\hline 0,900 & 225 & $3,11 \cdot 10^{-3}$ \\
\hline 0,600 & 740 & $3,00 \cdot 10^{-3}$ \\
\hline 0,400 & 2900 & $4,12 \cdot 10^{-3}$ \\
\hline
\end{tabular}

A partir dos valores das Tabela 2, Tabela 3 e Tabela 4, e adotando uma metodologia inversa de identificação paramétrica, os seguintes valores de porosidade inicial $f_{o}$ foram encontrados, assumindo que a vida experimental observada pode ser determinada numericamente a partir de um valor de porosidade crítica $f_{c}=$ 0,017; 0,013; e 0,015, respectivamente para os aços 304; S460N e alumínio 6061-T6. A Tabela 5 resume todas as propriedades dos materiais para o modelo de Gurson com endurecimento cinemático de Armstrong-Frederick, considerando a porosidade inicial igual ao valor médio apresentado para os ciclos experimentais definidos. 
Tabela 5. Resumo das propriedades dos materiais envolvidos na análise.

\begin{tabular}{|c|c|c|c|}
\cline { 2 - 4 } \multicolumn{1}{c|}{} & \multicolumn{3}{c|}{ Materiais } \\
\hline Parâmetros & $\mathbf{3 0 4}$ (Itoh, 2001) & S460N (Jiang et al, 2007) & 6061-T6 (Itoh, 2001) \\
\hline$H^{k}(\mathrm{MPa})$ & 78079 & 78166 & 14781 \\
\hline$b$ & 328 & 365 & 418 \\
\hline$f_{o}$ & $3,26 \cdot 10^{-5}$ & $1,64 \cdot 10^{-4}$ & $3,41 \cdot 10^{-3}$ \\
\hline$f_{c}$ & 0,017 & 0,013 & 0,015 \\
\hline
\end{tabular}

\subsection{TRAJETÓRIAS PROPORCIONAIS E NÃO PROPORCIONAIS}

Baseando-se nos estudos de Jiang, et al. (2007), e Itoh (2001), objetiva-se estabelecer trajetórias e carregamentos que permitam a simulação de vida à fadiga dos materiais em diferentes situações, sendo que foram analisados quatro históricos de carregamento distintos para o aço $\mathrm{S} 460 \mathrm{~N}$, Tabela 6 , e três históricos de carregamento distintos para o aço 304 e a liga de alumínio 6061-T6,Tabela 7.

Para ambos os materiais, os autores consideram trajetórias uniaxiais, multiaxiais proporcionais e multiaxiais não proporcionais, estando as rotinas de simulação destes carregamentos implementadas em FORTRAN.

Tabela 6. Históricos de carregamento impostos para o aço S460N. Fonte: Jiang et al., 2007.

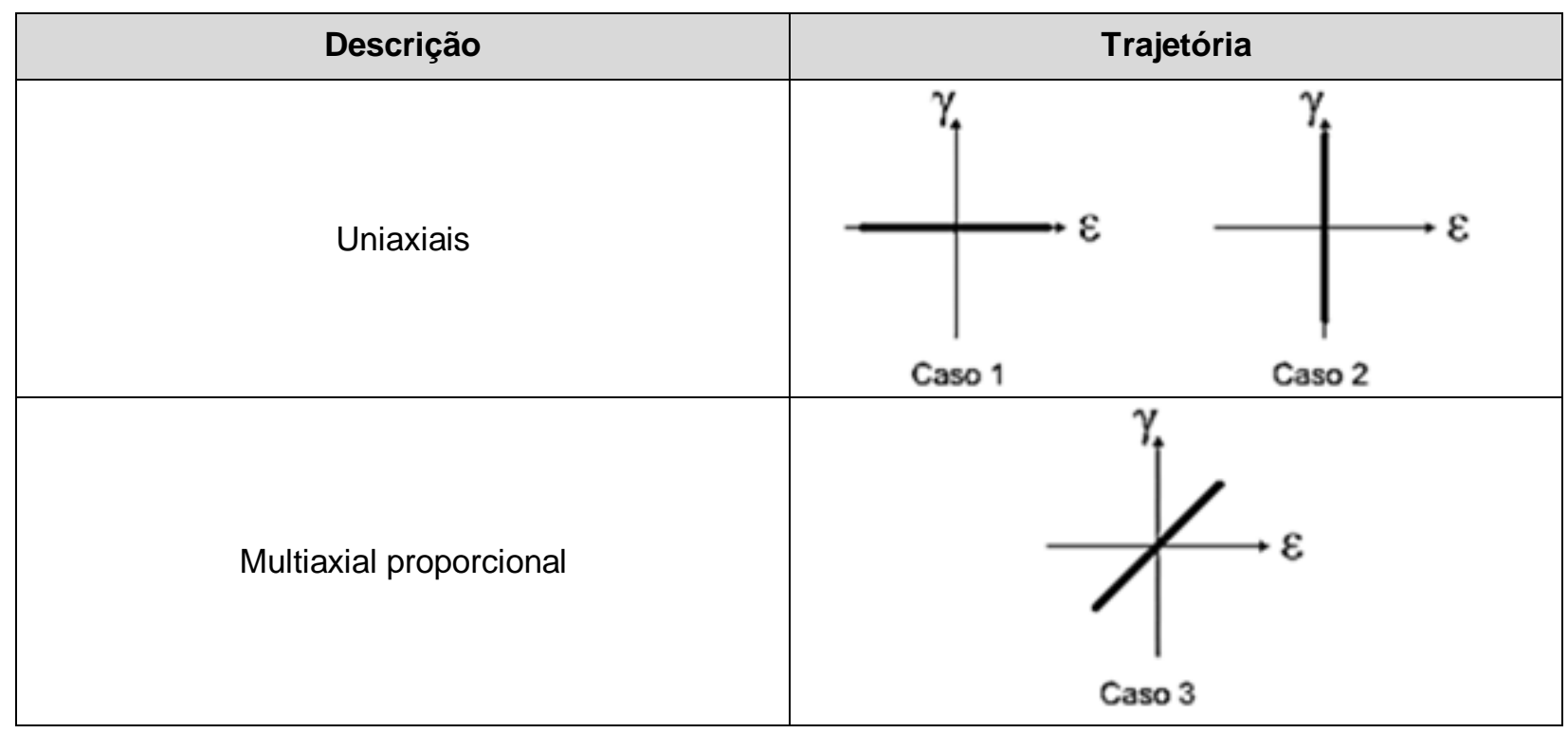




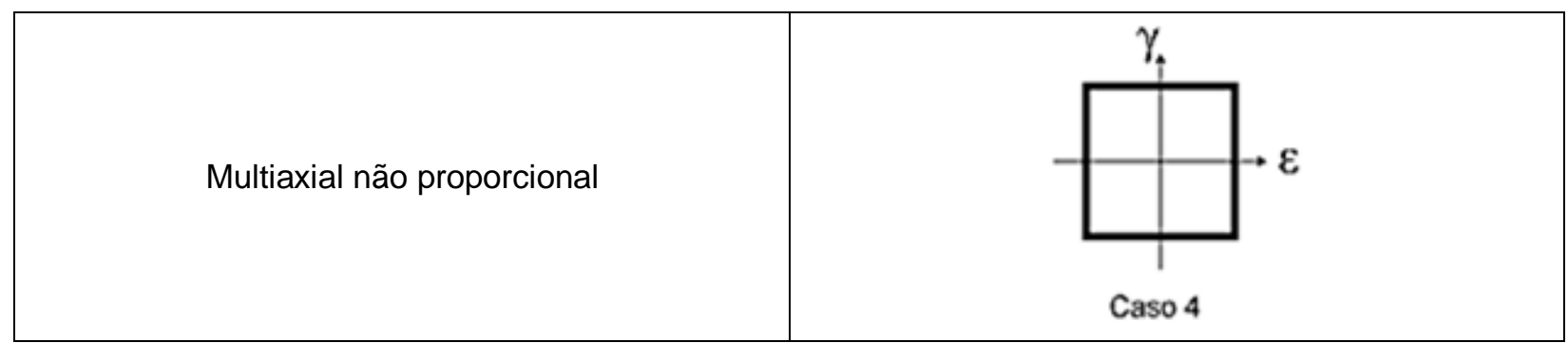

Tabela 7. Históricos de carregamento impostos para o aço 304 e a liga de alumínio 6061-T6. Fonte: Itoh, 2001.

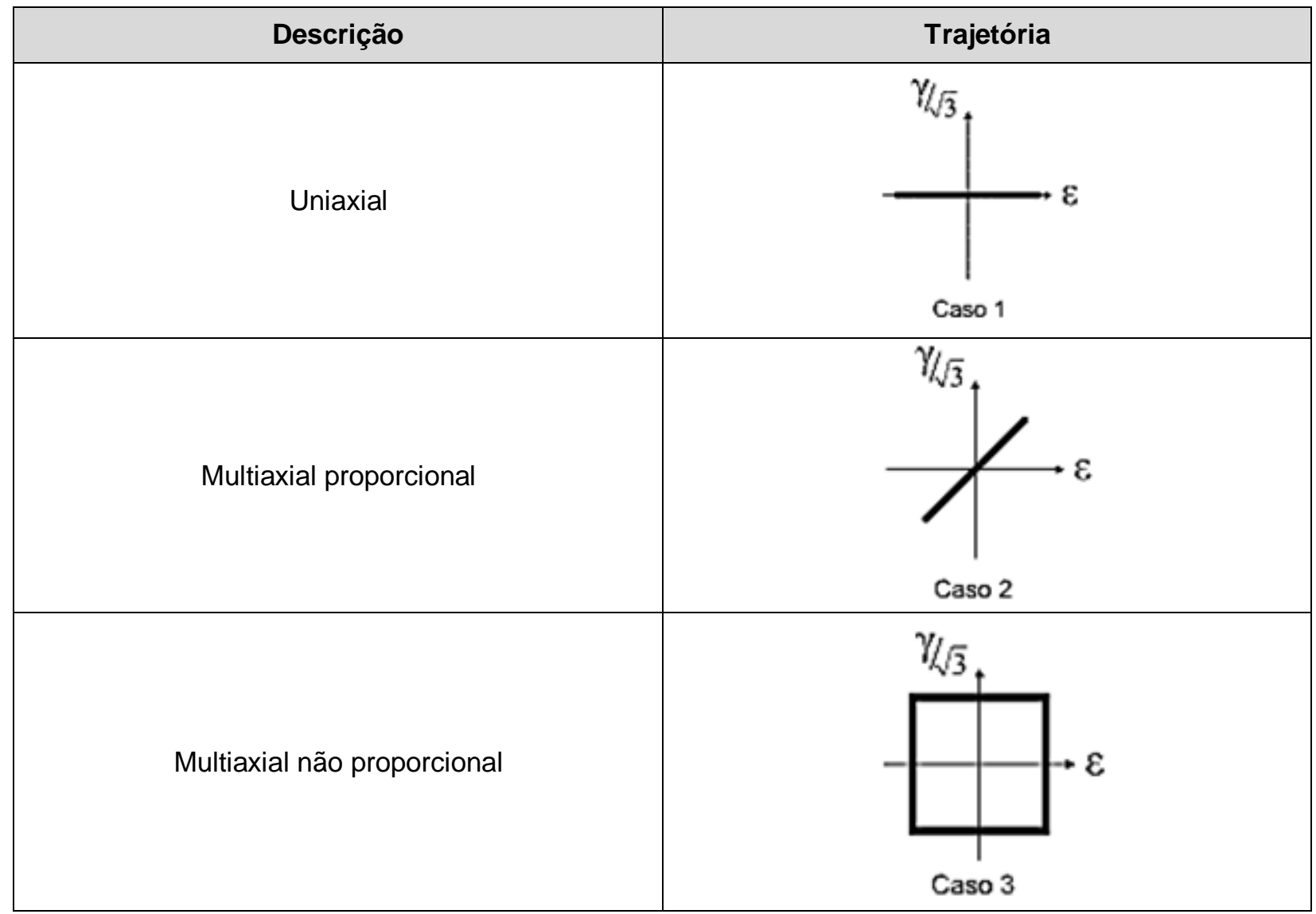

\subsubsection{Previsão de vida a fadiga para trajetórias uniaxiais}

A partir das trajetórias uniaxiais apresentadas nos casos 1 e 2 para o aço $5460 \mathrm{~N}$ (Tabela 6) e da trajetória uniaxial do caso 1 para os o aço 304 e alumínio 6061-T6 (Tabela 7 ), as seguintes estimativas de vida a fadiga foram determinadas através do modelo da micromecânica de defeito de Gurson, resumidas nas Tabela 8, Tabela 9 e Tabela 10 e ilustradas nas Figura 16, Figura 17 e Figura 18. 
Tabela 8. Análise comparativa da vida numérica para vida experimental em diferentes trajetórias uniaxiais. Aço 304 (Itoh, 2001).

\begin{tabular}{|c|c|c|c|c|}
\hline \multicolumn{5}{|c|}{ Aço 304 (Itoh, 2001) } \\
\hline \multirow{3}{*}{ Caso } & $\boldsymbol{\varepsilon}_{\boldsymbol{a}}$ & $\boldsymbol{\gamma}_{\boldsymbol{a}}$ & $\boldsymbol{N}_{\boldsymbol{E X P}}$ & $\boldsymbol{N}_{\text {NUM }}$ \\
\hline \multirow{3}{*}{1} & 0,600 & 0 & 690 & 1146 \\
\cline { 2 - 5 } & 0,500 & 0 & 1500 & 2488 \\
\cline { 2 - 5 } & 0,400 & 0 & 7100 & 5459 \\
\cline { 2 - 5 } & 0,325 & 0 & 23400 & 10246 \\
\cline { 2 - 5 } & 0,250 & 0 & 49000 & 20287 \\
\hline
\end{tabular}

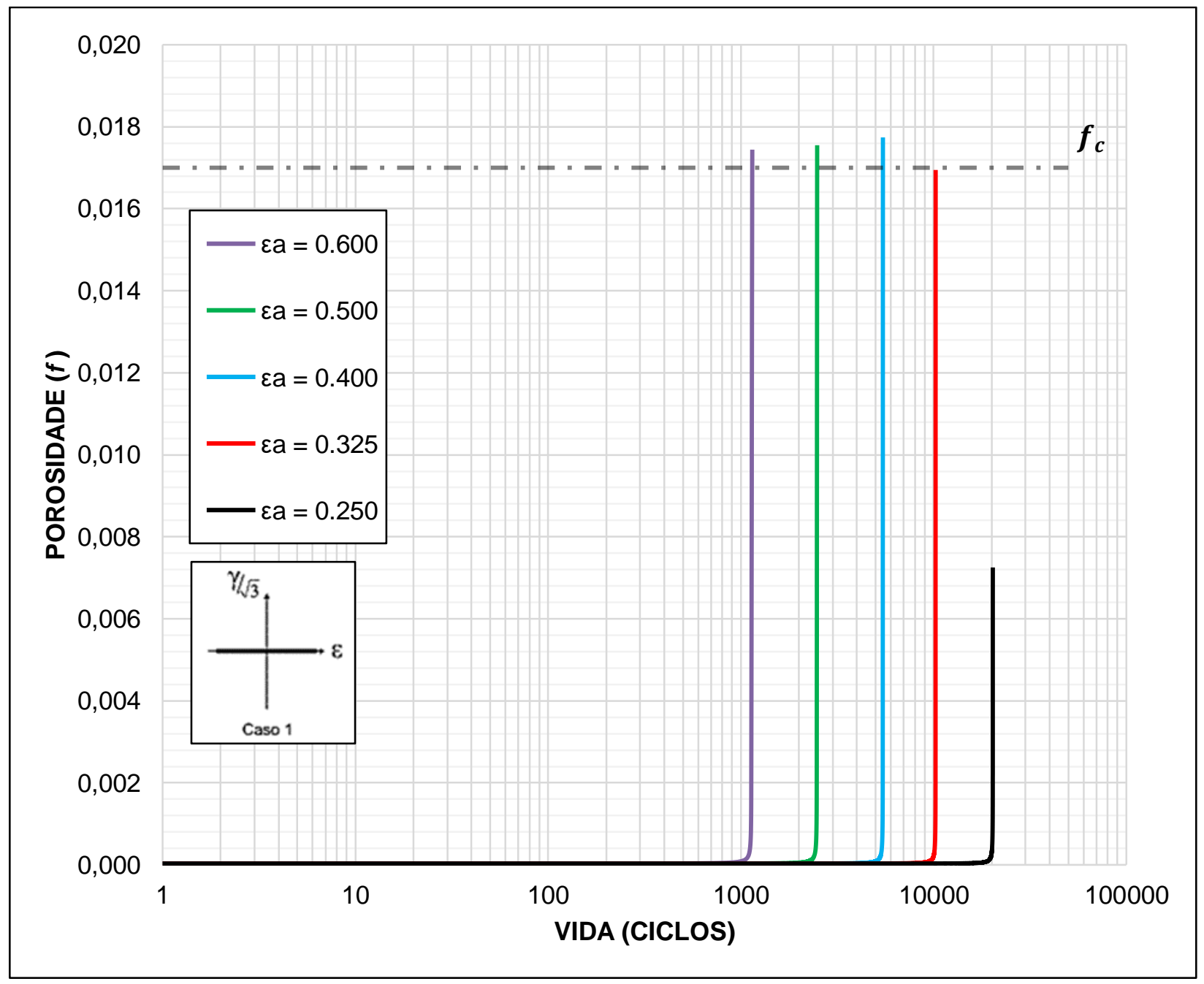

Figura 16. Evolução da porosidade em função do ciclo de vida do Aço 304. 
Tabela 9. Análise comparativa da vida numérica para vida experimental em diferentes trajetórias uniaxiais. Aço S460N (Jiang, et al. 2007).

\begin{tabular}{|c|c|c|c|c|}
\hline \multicolumn{5}{|c|}{ Aço S460N (Jiang, et al. 2007) } \\
\hline \multirow{3}{*}{ Caso } & $\boldsymbol{\varepsilon}_{\boldsymbol{a}}$ & $\boldsymbol{\gamma}_{\boldsymbol{a}}$ & $\boldsymbol{N}_{\boldsymbol{E X P}}$ & $\boldsymbol{N}_{\boldsymbol{N} \text { UM }}$ \\
\hline \multirow{3}{*}{1} & 0,500 & 0 & 1600 & 2302 \\
\cline { 2 - 5 } & 0,330 & 0 & 7690 & 6884 \\
\cline { 2 - 5 } & 0,220 & 0 & 33100 & 21991 \\
\hline \multirow{3}{*}{2} & 0 & 1,00 & 1820 & Infinita \\
\cline { 2 - 5 } & 0 & 0,45 & 23000 & Infinita \\
\cline { 2 - 5 } & 0 & 0,43 & 38250 & Infinita \\
\hline
\end{tabular}

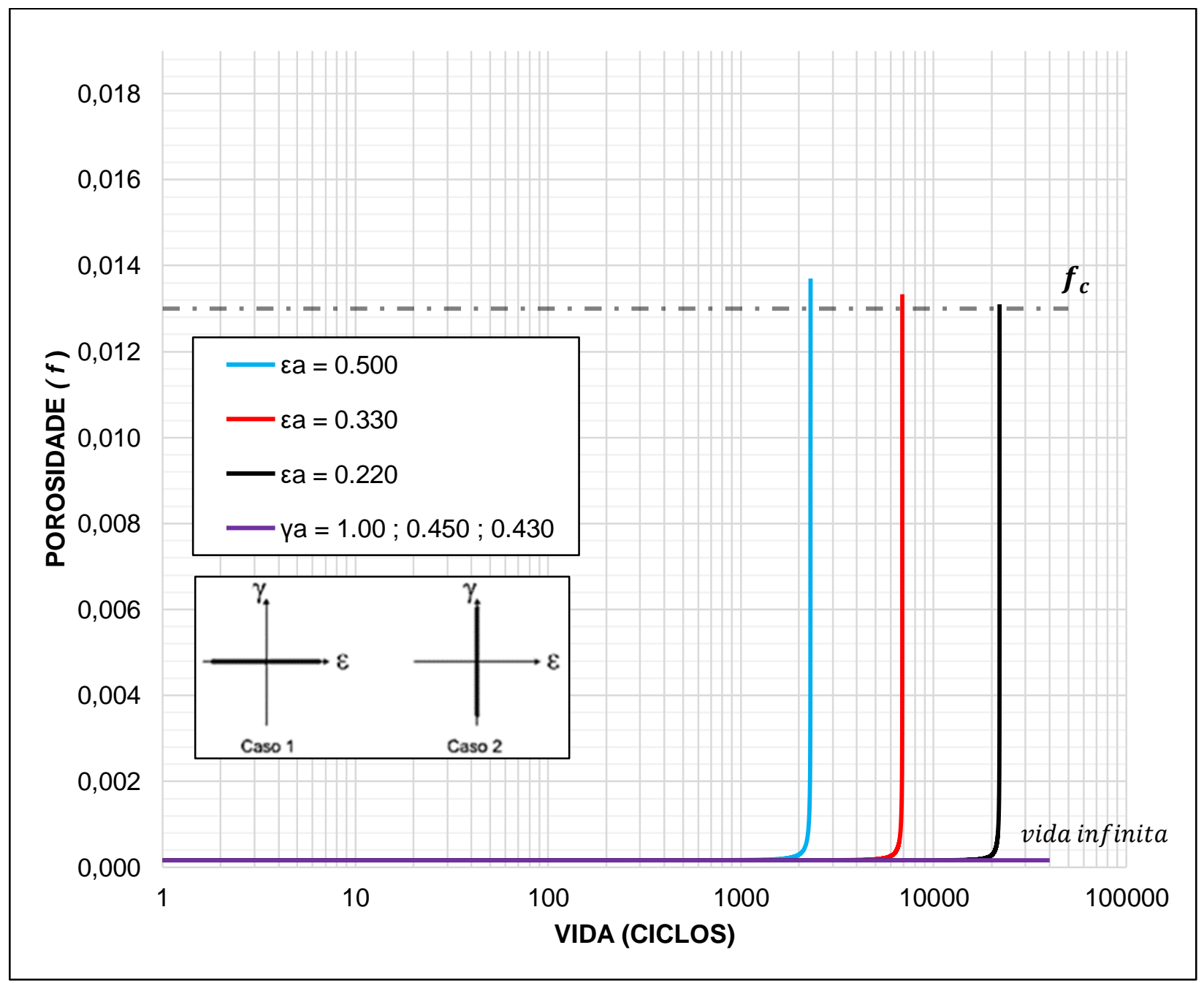

Figura 17. Evolução da porosidade em função do ciclo de vida do Aço S460N. 
Tabela 10. Análise comparativa da vida numérica para vida experimental em diferentes trajetórias uniaxiais. Alumínio 6061-T6 (Itoh, 2001).

\begin{tabular}{|c|c|c|c|c|}
\hline \multicolumn{5}{|c|}{ Alumínio 6061-T6 (Itoh, 2001) } \\
\hline \multirow{2}{*}{ Caso } & $\boldsymbol{\varepsilon}_{\boldsymbol{a}}$ & $\boldsymbol{\gamma}_{\boldsymbol{a}}$ & $\boldsymbol{N}_{\boldsymbol{E X P}}$ & $\boldsymbol{N}_{\boldsymbol{N} \text { UM }}$ \\
\hline \multirow{3}{*}{1} & 0,90 & 0 & 225 & 205 \\
\cline { 2 - 5 } & 0,60 & 0 & 740 & 648 \\
\cline { 2 - 5 } & 0,40 & 0 & 2900 & 3538 \\
\hline
\end{tabular}

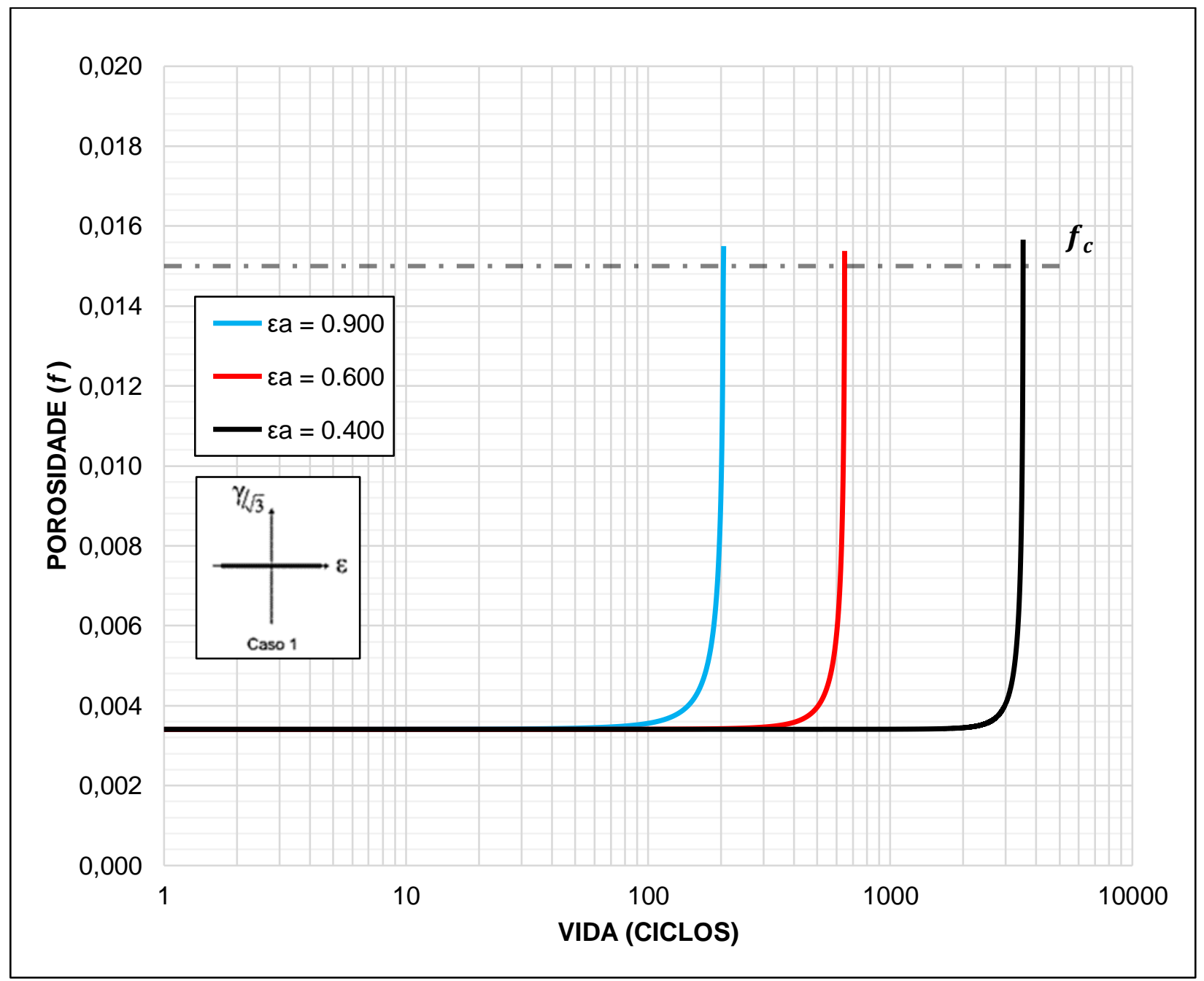

Figura 18. Evolução da porosidade em função do ciclo de vida do Alumínio 6061-T6. 
Observa-se que para valores elevados de amplitude de deformação a porosidade atinge o seu valor crítico para uma vida próxima da vida observada experimentalmente, enquanto que, para amplitudes menores de deformação, onde o nível de deformação plástica macroscópica é baixo, o valor de porosidade crítica só é atingido para níveis elevados de vida calculada, estando distante dos valores experimentalmente observados. Para alguns casos, observa-se que a evolução da porosidade não atinge o valor crítico.

Tal análise pode ser conferida através das amplitudes de deformação $\varepsilon_{a}=0,250$ para o aço 304 (Figura 16) e $\varepsilon_{a}=0,230$ para o aço S406N (Figura 17), as quais não atingem o valor de porosidade crítica por conta da não ocorrência de um nível de plasticidade que pudesse ter sido capturado pelo modelo de Gurson (1977).

Para o caso do alumínio 6061-T6, Tabela 10 e Figura 18, observa-se que este demonstrou boa convergência para o valor da porosidade crítica definida, mesmo para casos de baixa amplitude de deformação, tal resultado pode ser justificado por, mesmo em baixas amplitudes, o referido material ainda demonstrar um nível de deformação plástica macroscópica elevado.

Pelo modelo original de Gurson (1977) não incluir os efeitos de distorção do vazio e, portanto, não permitir a evolução da porosidade através de carregamentos predominantemente cisalhantes, nota-se que para as amplitudes de deformação aplicadas no Aço S460N, Tabela 9 e Figura 17, quando aplicados os carregamentos cisalhantes do Caso 2, a porosidade inicial não evolui e o material apresenta vida infinita, o que é dado como inconsistente do ponto de vista físico.

Por fim, a simulação dos casos executados para trajetórias uniaxiais normais traz boa confiabilidade para a análise da precisão do modelo da micromecânica do defeito de Gurson (1977) para previsão de vida a fadiga, considerando a faixa de vida estimada entre metade e duas vezes a vida observada. Nessa linha, as Figura 19, Figura 20 e Figura 21 apresentam os gráficos de comparação entre a vida em fadiga obtida experimentalmente e a vida estimada pelo modelo de Gurson para os aços 304 e S460N e para o alumínio 6061-T6. 


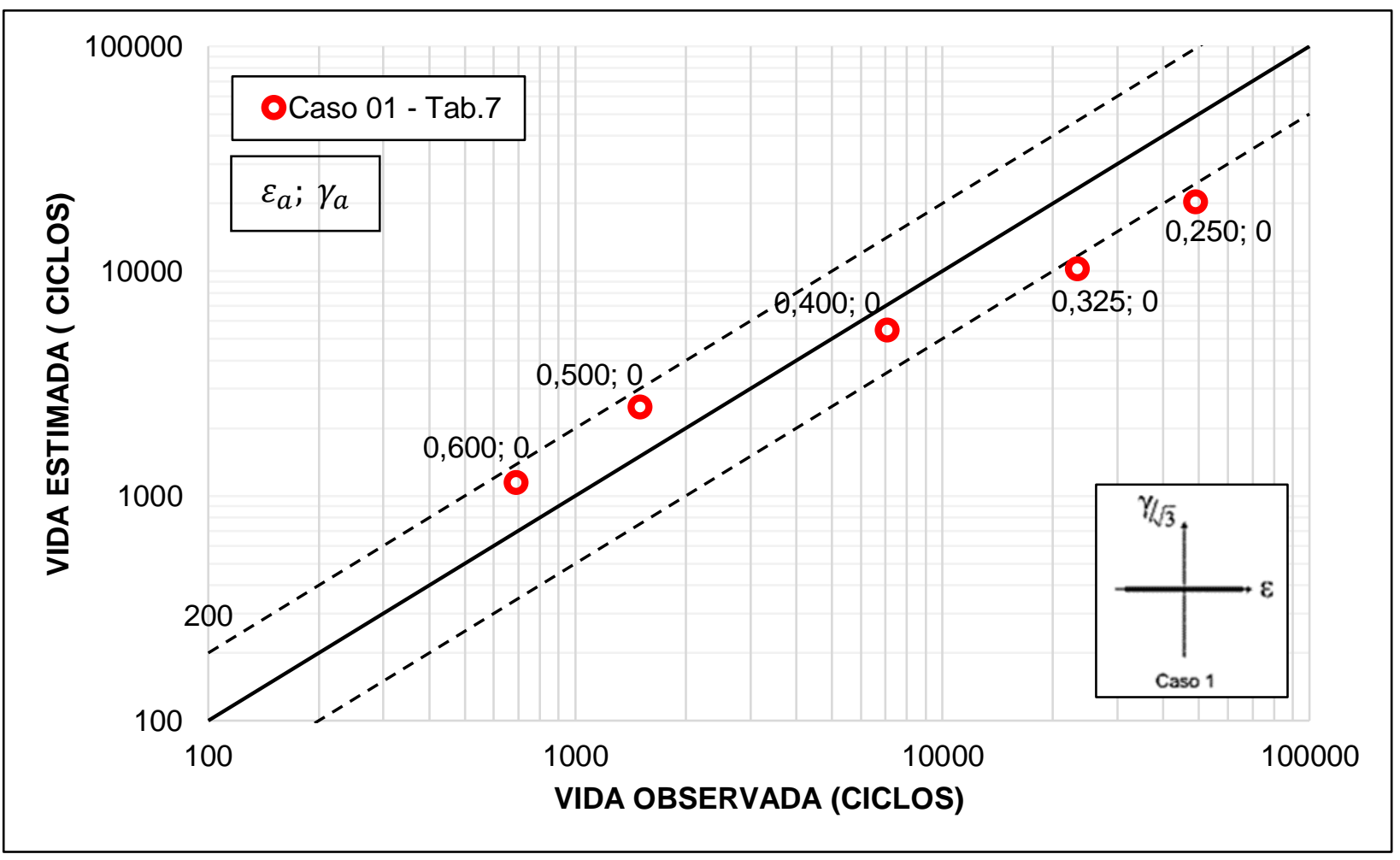

Figura 19. Comparação entre a vida observada experimentalmente e a vida estimada numericamente pelo modelo de Gurson. Aço 304 em trajetória uniaxial.

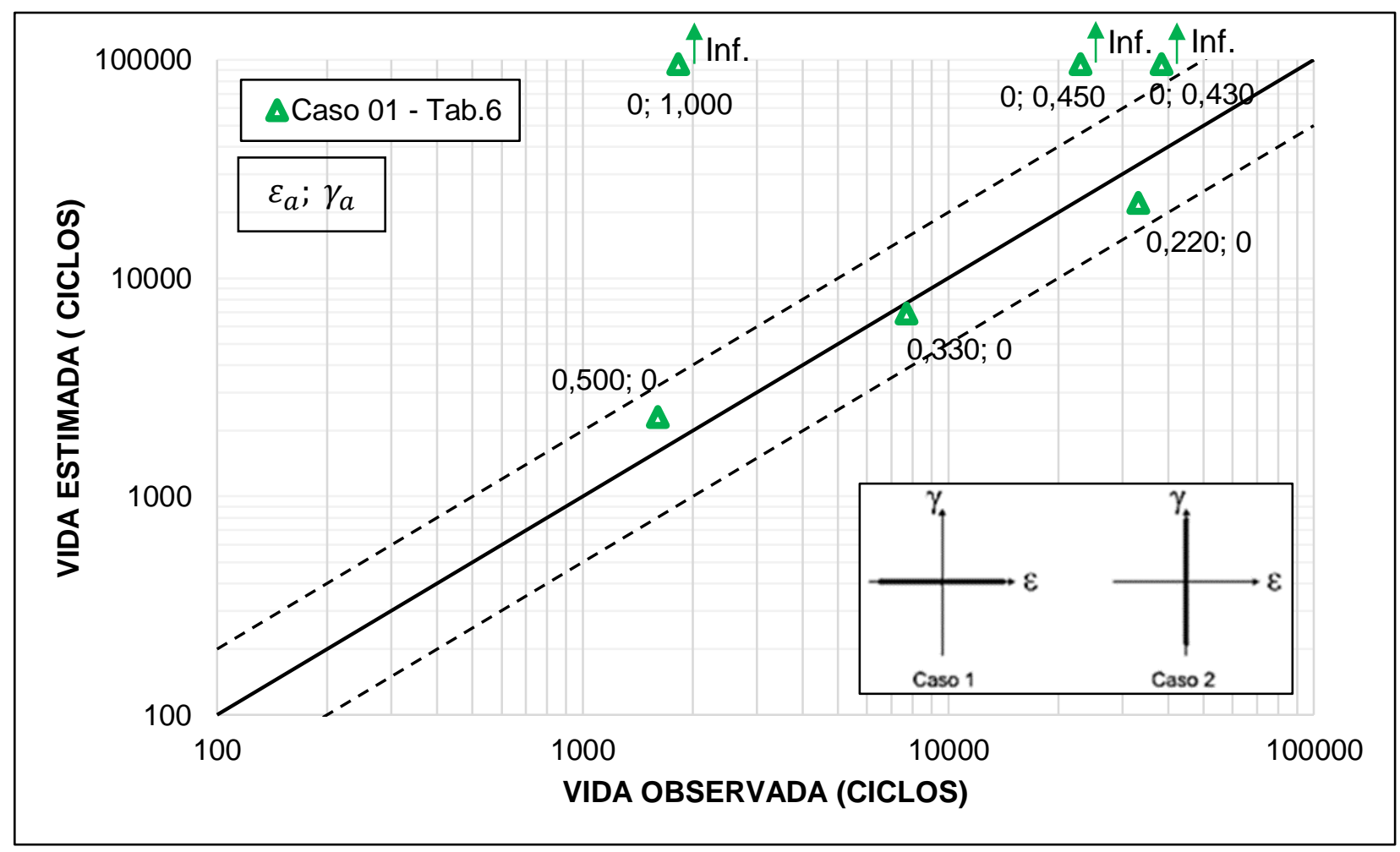

Figura 20. Comparação entre a vida observada experimentalmente e a vida estimada numericamente pelo modelo de Gurson. Aço S460N em trajetória uniaxial. 


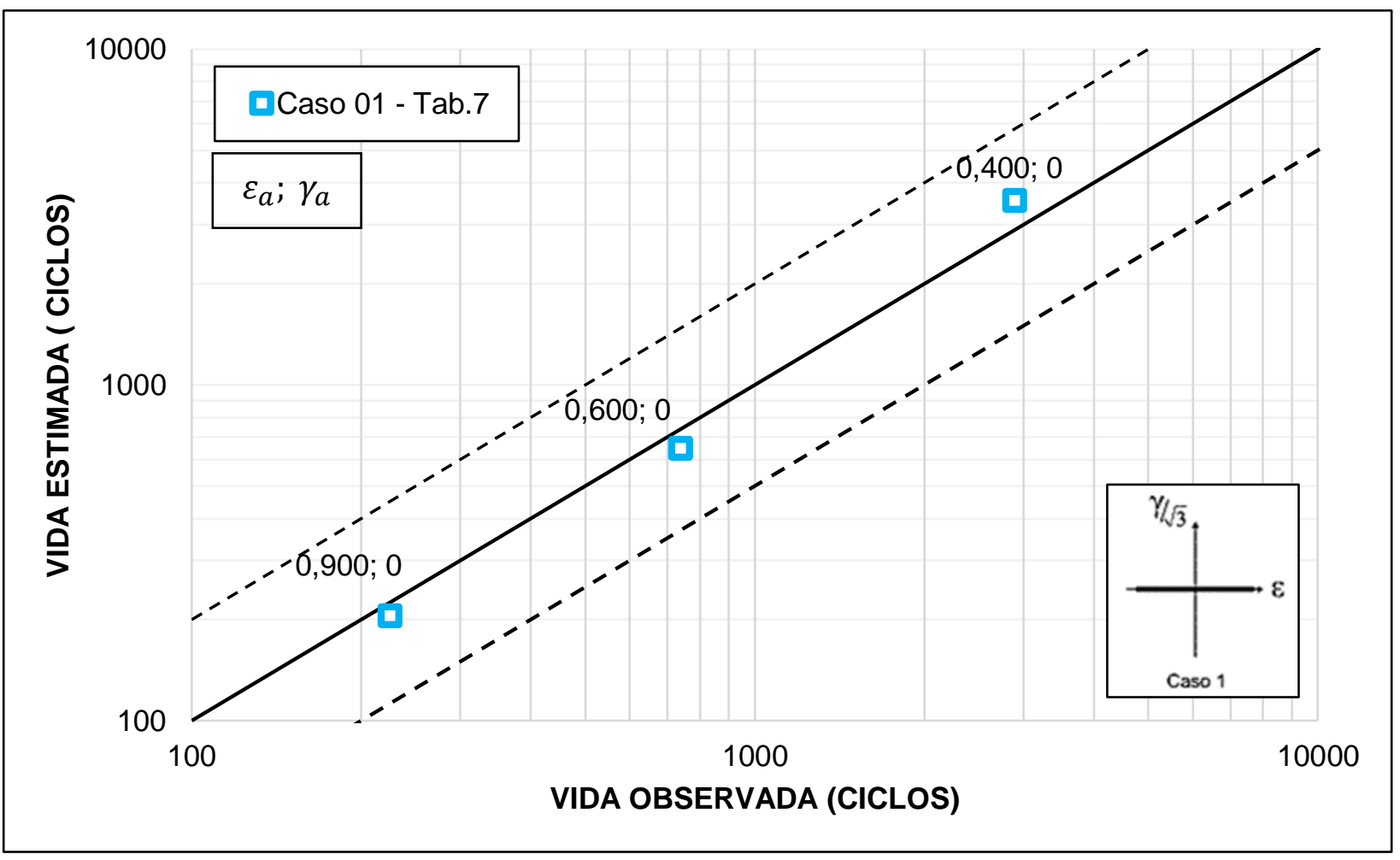

Figura 21. Comparação entre a vida observada experimentalmente e a vida estimada numericamente pelo modelo de Gurson. Alumínio 6061-T6 em trajetória uniaxial.

\subsubsection{Previsão de vida a fadiga para trajetórias multiaxiais proporcionais}

A partir da trajetória multiaxial proporcional apresentada no caso 3 para o aço S460N (Tabela 6) e do caso 2 para os o aço 304 e alumínio 6061-T6 (Tabela 7), as seguintes estimativas de vida a fadiga foram determinadas através do modelo da micromecânica de defeito de Gurson, resumidas na Tabela 11, Tabela 12 e Tabela 13 e ilustradas nas Figura 22, Figura 23 e Figura 24. 
Tabela 11. Análise comparativa da vida numérica para vida experimental em diferentes trajetórias multiaxiais proporcionais. Aço 304 (Itoh, 2001).

\begin{tabular}{|c|c|c|c|c|}
\hline \multicolumn{5}{|c|}{ Aço 304 (Itoh, 2001) } \\
\hline Caso & $\boldsymbol{\varepsilon}_{\boldsymbol{a}}$ & $\boldsymbol{\gamma}_{\boldsymbol{a}}$ & $\boldsymbol{N}_{\boldsymbol{E X P}}$ & $\boldsymbol{N}_{\text {NUM }}$ \\
\hline \multirow{2}{*}{2} & 0,250 & 0,435 & 17500 & 9779 \\
\cline { 2 - 5 } & 0,400 & 0,695 & 3200 & 2708 \\
\hline
\end{tabular}

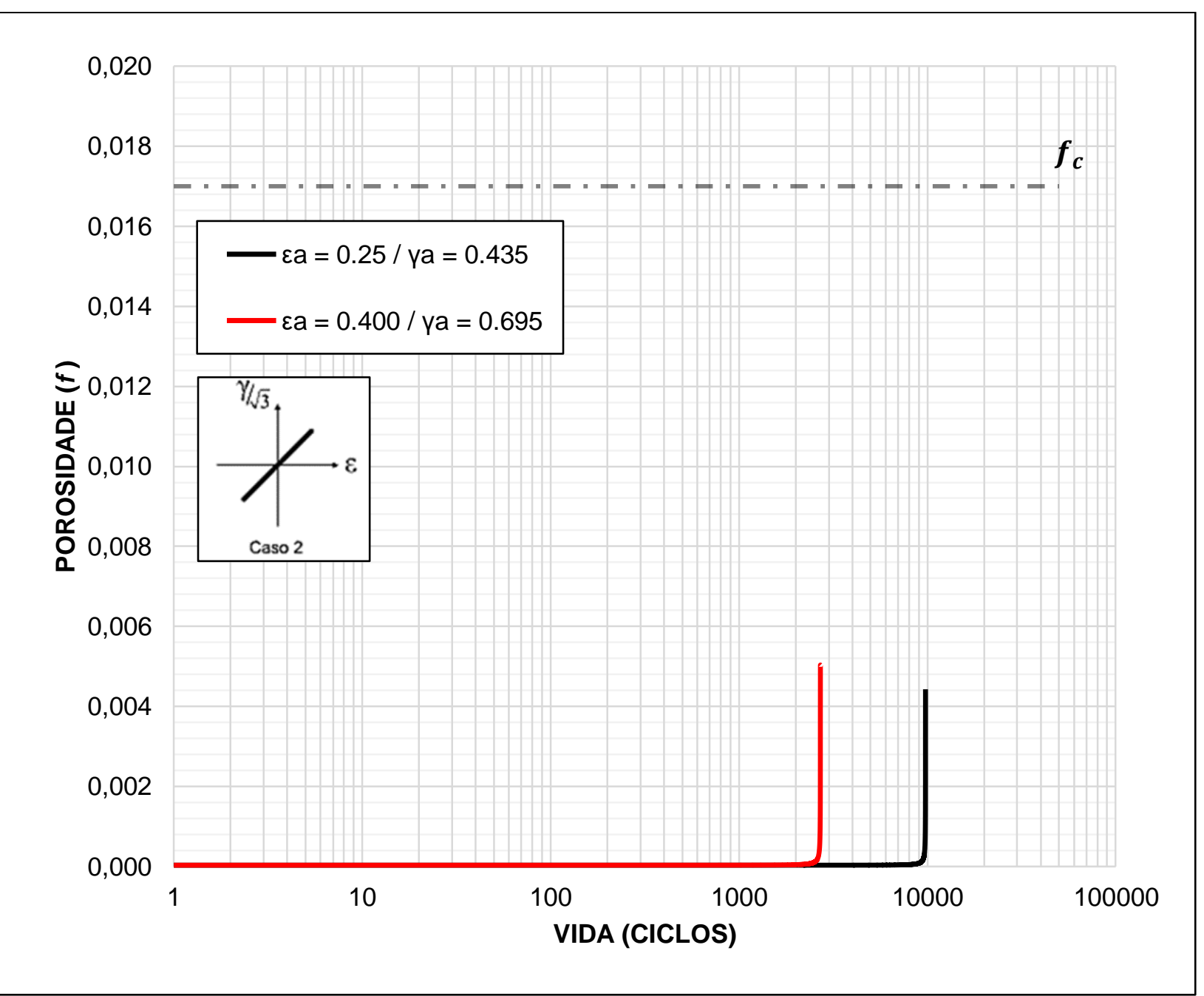

Figura 22. Evolução da porosidade em função do ciclo de vida do Aço 304. 
Tabela 12. Análise comparativa da vida numérica para vida experimental em diferentes trajetórias multiaxiais proporcionais. Aço S460N (Jiang, et al. 2007).

\begin{tabular}{|c|c|c|c|c|}
\hline \multicolumn{5}{|c|}{ Aço S460N (Jiang, et al. 2007) } \\
\hline \multirow{2}{*}{ Caso } & $\boldsymbol{\varepsilon}_{\boldsymbol{a}}$ & $\boldsymbol{\gamma}_{\boldsymbol{a}}$ & $\boldsymbol{N}_{\boldsymbol{E X P}}$ & $\boldsymbol{N}_{\boldsymbol{N} \text { UM }}$ \\
\hline \multirow{3}{*}{3} & 0,173 & 0,30 & 31100 & 14589 \\
\cline { 2 - 5 } & 0,104 & 0,18 & 521000 & 134720 \\
\cline { 2 - 5 } & 0,144 & 0,25 & 130300 & 25224 \\
\hline
\end{tabular}

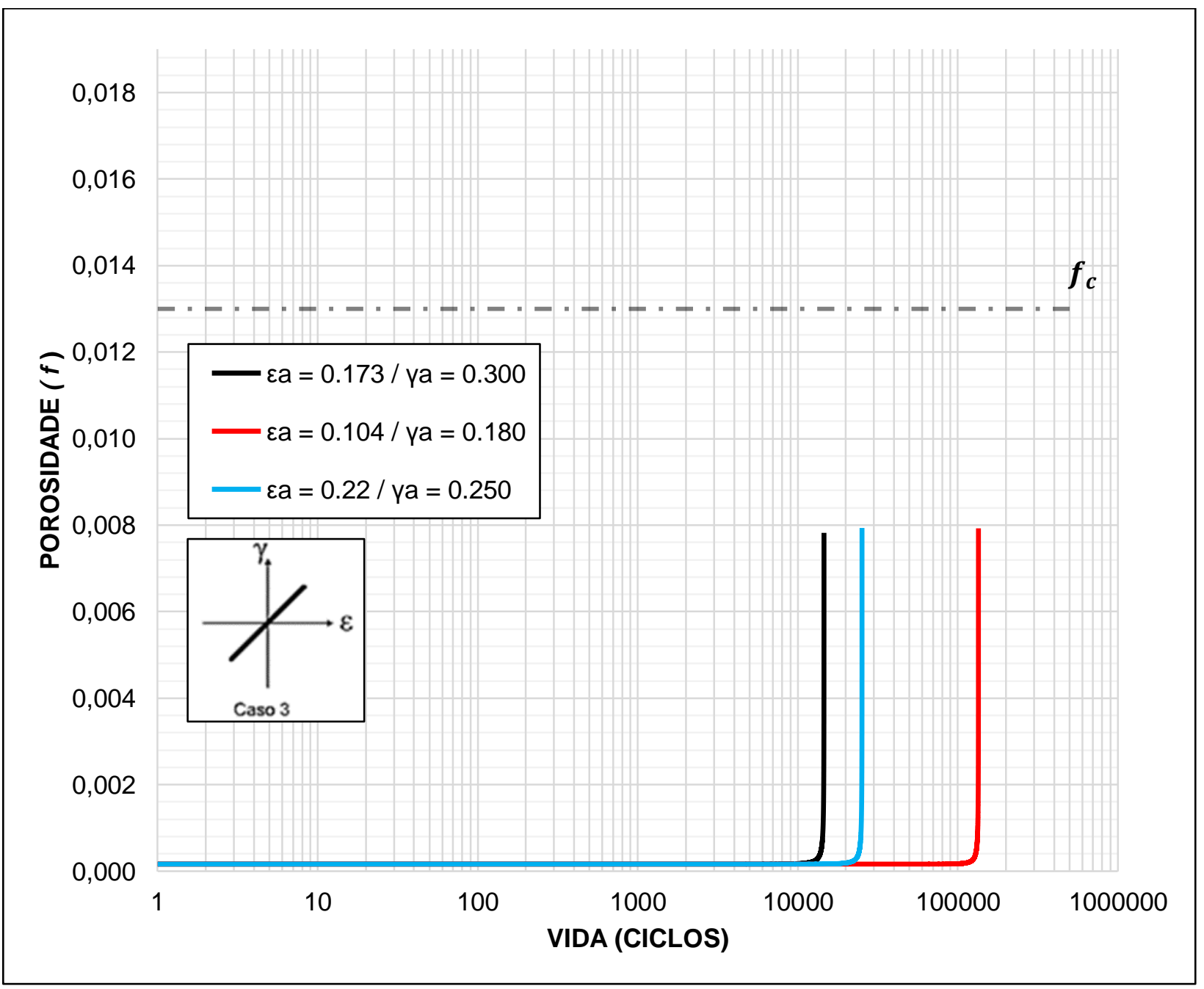

Figura 23. Evolução da porosidade em função do ciclo de vida do Aço S460N. 
Tabela 13. Análise comparativa da vida numérica para vida experimental em trajetória multiaxial proporcional. Alumínio 6061-T6 (Itoh, 2001).

\begin{tabular}{|c|c|c|c|c|}
\hline \multicolumn{5}{|c|}{ Alumínio 6061-T6 (Itoh, 2001) } \\
\hline Caso & $\varepsilon_{\boldsymbol{a}}$ & $\gamma_{\boldsymbol{a}}$ & $\boldsymbol{N}_{\boldsymbol{E X P}}$ & $\boldsymbol{N}_{\boldsymbol{N} \text { UM }}$ \\
\hline 2 & 0,600 & 0,735 & 970 & 327 \\
\hline
\end{tabular}

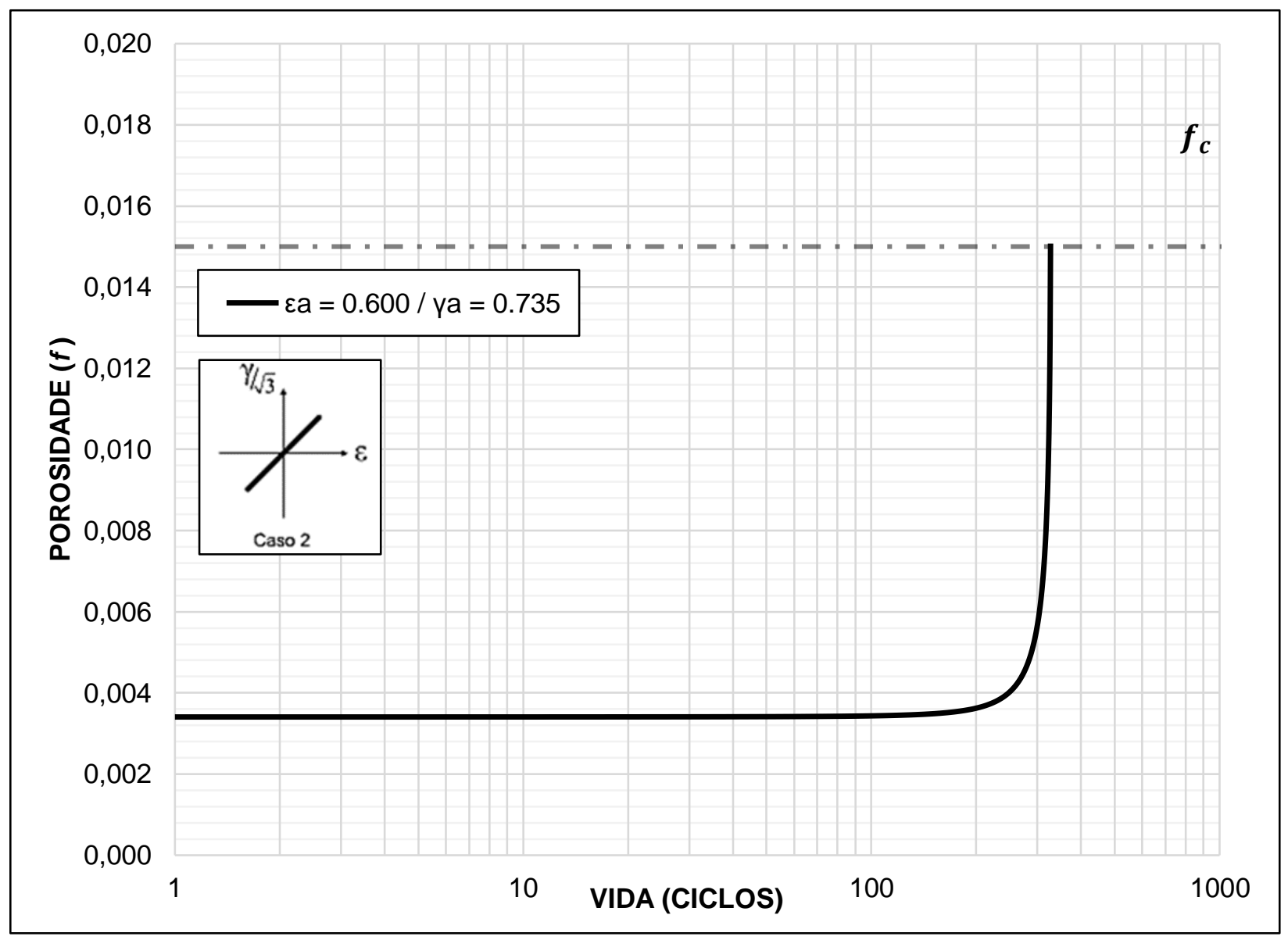

Figura 24. Evolução da porosidade em função do ciclo de vida do Alumínio 6061-T6.

Observa-se que, conforme discutido para as trajetórias uniaxiais, para valores onde o nível de deformação plástica macroscópica é baixo, mesmo com o valor de porosidade crítica atingido, os valores calculados estão distantes dos experimentalmente observados. Essa ocorrência possivelmente é ocasionada pela característica do modelo de Gurson (1977) não incluir os efeitos de distorção do vazio e, portanto, não permitir a evolução da porosidade através de carregamentos predominantemente cisalhantes.

Tal análise pode ser conferida através das amplitudes de deformação $\varepsilon_{a}=0,250$ para o aço 304 (Figura 16) e $\varepsilon_{a}=0,230$ para o aço S406N (Figura 17), as quais não 
atingem o valor de porosidade crítica por conta da não ocorrência de um nível de plasticidade que pudesse ter sido capturado pelo modelo de Gurson (1977).

Para o caso dos aços 304 e S460N, Tabela 11 e Tabela 12, observa-se que estes, mesmo não atingindo o valor de porosidade crítica, demonstraram boa convergência para o valor da porosidade similar para as faixas de amplitudes aplicadas, o que pode ser observado como a definição de um ponto de porosidade crítica estabelecido pelo modelo de Gurson (1977) quando desprezadas as distorções causadas pelas forças cisalhantes.

Por fim, a simulação dos casos executados para trajetórias multiaxiais não agregou boa confiabilidade para a análise da precisão do modelo da micromecânica do defeito de Gurson (1977) para previsão de vida a fadiga, considerando a faixa de vida estimada entre metade e duas vezes a vida observada. Nessa linha, as Figura 25, Figura 26 e Figura 27 apresentam os gráficos de comparação entre a vida em fadiga obtida experimentalmente e a vida estimada pelo modelo de Gurson para os aços 304 e S460N e para o alumínio 6061-T6.

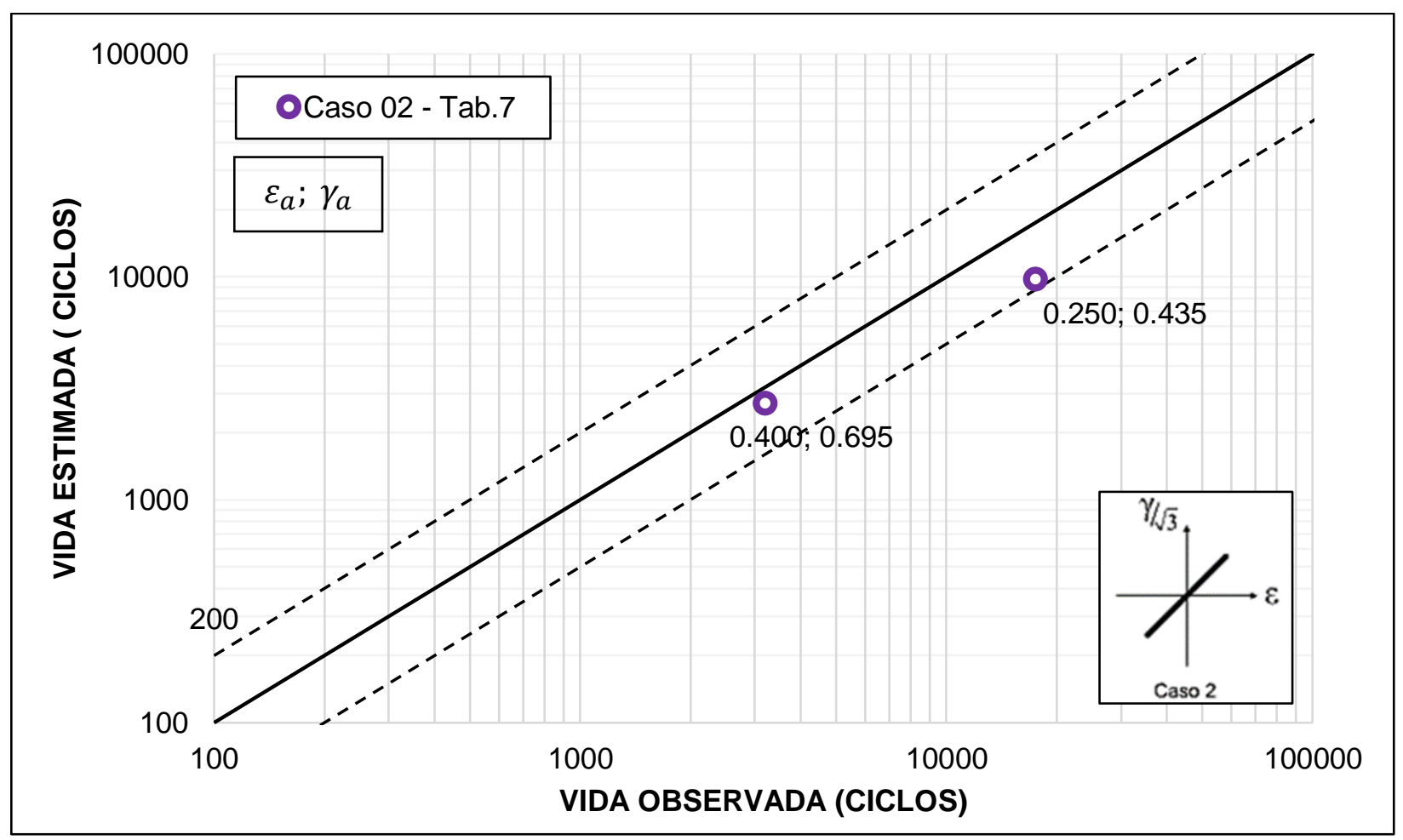

Figura 25. Comparação entre a vida observada experimentalmente e a vida estimada numericamente pelo modelo de Gurson. Aço 304 em trajetória multiaxial proporcional. 


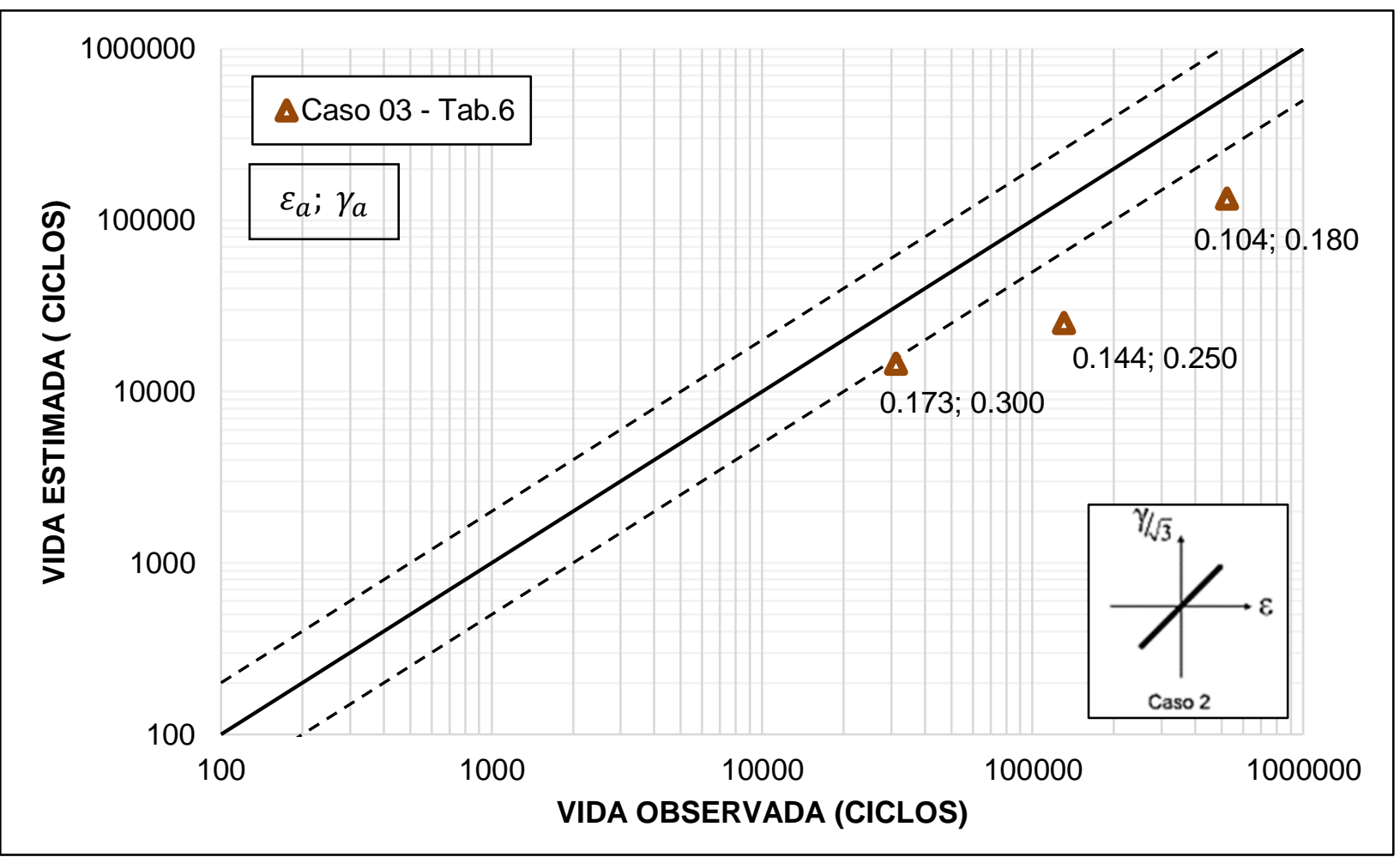

Figura 26. Comparação entre a vida observada experimentalmente e a vida estimada numericamente pelo modelo de Gurson. Aço $\mathbf{S} 460 \mathrm{~N}$ em trajetória multiaxial proporcional.

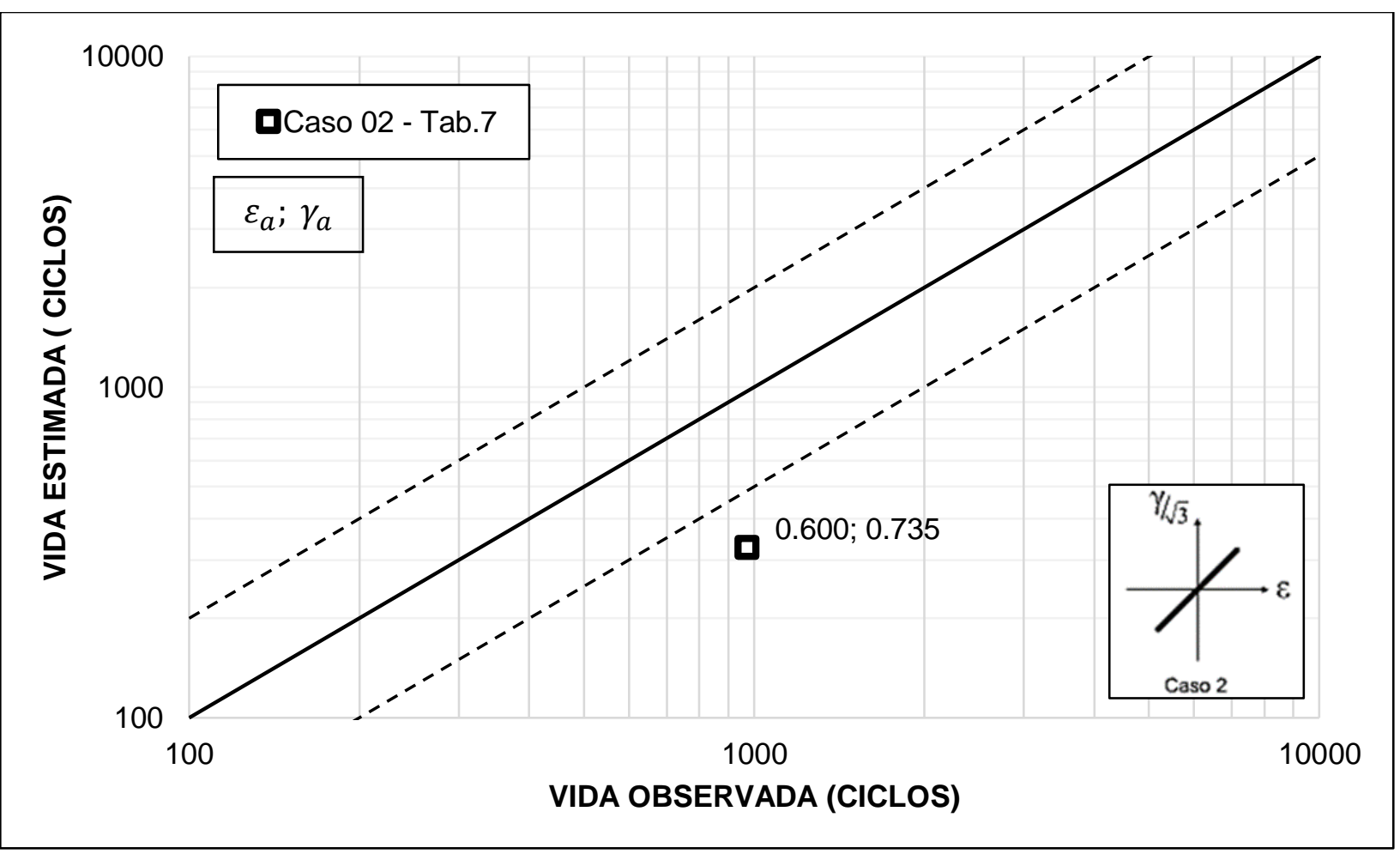

Figura 27. Comparação entre a vida observada experimentalmente e a vida estimada numericamente pelo modelo de Gurson. Alumínio 6061-T6 em trajetória multiaxial proporcional. 


\subsubsection{Previsão de vida a fadiga para trajetórias multiaxiais não proporcionais}

A partir da trajetória multiaxial não proporcionais apresentada no caso 4 para o aço S460N (Tabela 6) e do caso 3 para os o aço 304 e alumínio 6061-T6 (Tabela 7), as seguintes estimativas de vida a fadiga foram determinadas através do modelo da micromecânica de defeito de Gurson, resumidas na Tabela 14, Tabela 15 e Tabela 16, ilustradas nas Figura 28, Figura 29 e Figura 30.

Tabela 14. Análise comparativa da vida numérica para vida experimental em diferentes trajetórias multiaxiais não proporcionais. Aço 304 (Itoh, 2001).

\begin{tabular}{|c|c|c|c|c|}
\hline \multicolumn{5}{|c|}{ Aço 304 (Itoh, 2001) } \\
\hline Caso & $\boldsymbol{\varepsilon}_{\boldsymbol{a}}$ & $\boldsymbol{\gamma}_{\boldsymbol{a}}$ & $\boldsymbol{N}_{\boldsymbol{E X P}}$ & $\boldsymbol{N}_{\boldsymbol{N} \text { UM }}$ \\
\hline \multirow{2}{*}{3} & 0,250 & 0,435 & 2600 & 4805 \\
\cline { 2 - 5 } & 0,400 & 0,695 & 320 & 1006 \\
\hline
\end{tabular}

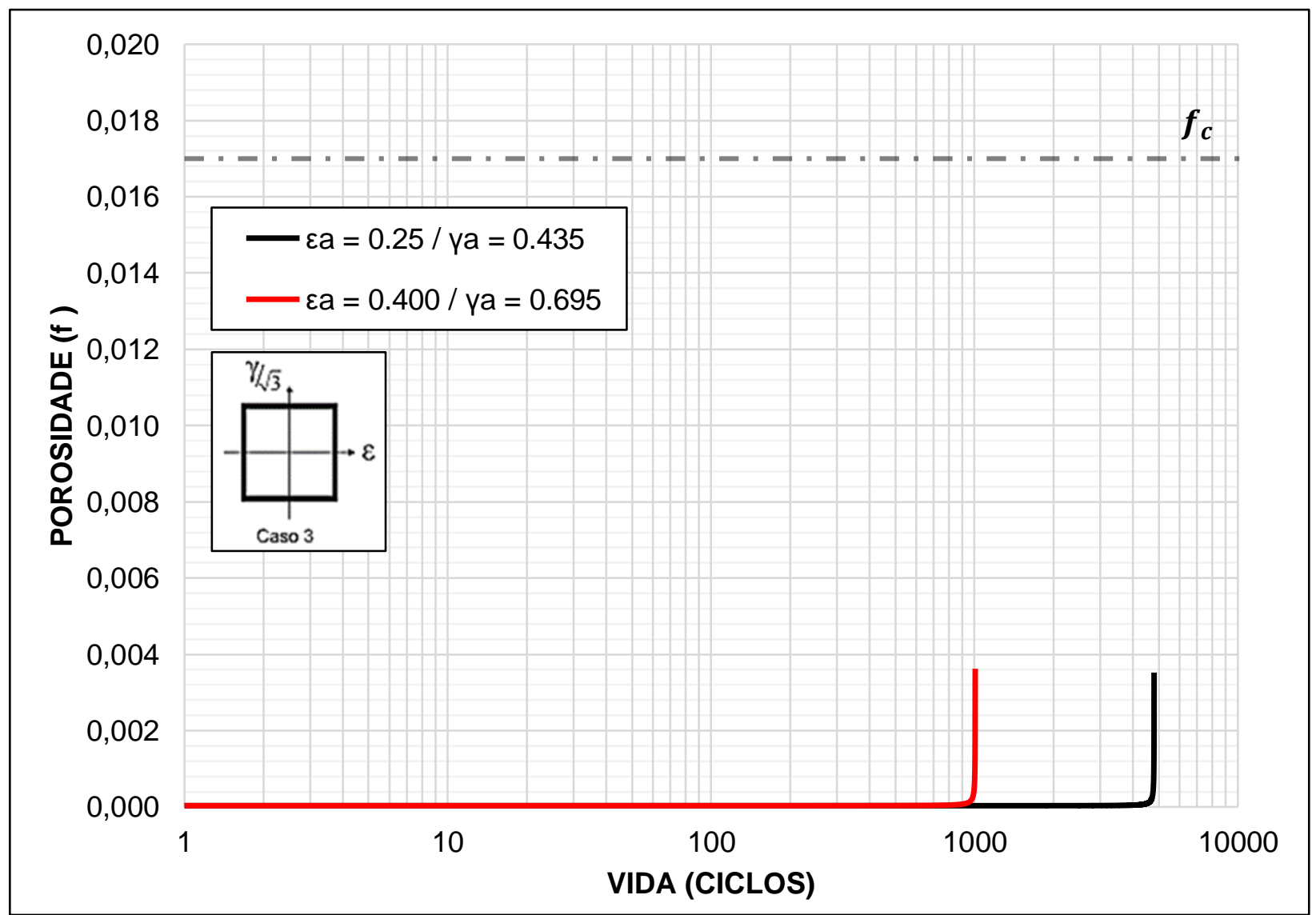

Figura 28. Evolução da porosidade em função do ciclo de vida do Aço 304. 
Tabela 15. Análise comparativa da vida numérica para vida experimental em diferentes trajetórias multiaxiais não proporcionais. Aço S460N (Jiang, et al. 2007).

\begin{tabular}{|c|c|c|c|c|}
\hline \multicolumn{5}{|c|}{ Aço S460N (Jiang, et al. 2007) } \\
\hline Caso & $\boldsymbol{\varepsilon}_{\boldsymbol{a}}$ & $\boldsymbol{\gamma}_{\boldsymbol{a}}$ & $\boldsymbol{N}_{\boldsymbol{E X P}}$ & $\boldsymbol{N}_{\boldsymbol{N} \text { UM }}$ \\
\hline \multirow{3}{*}{4} & 0,173 & 0,30 & 6730 & Infinita \\
\cline { 2 - 5 } & 0,144 & 0,25 & 18000 & Infinita \\
\hline
\end{tabular}

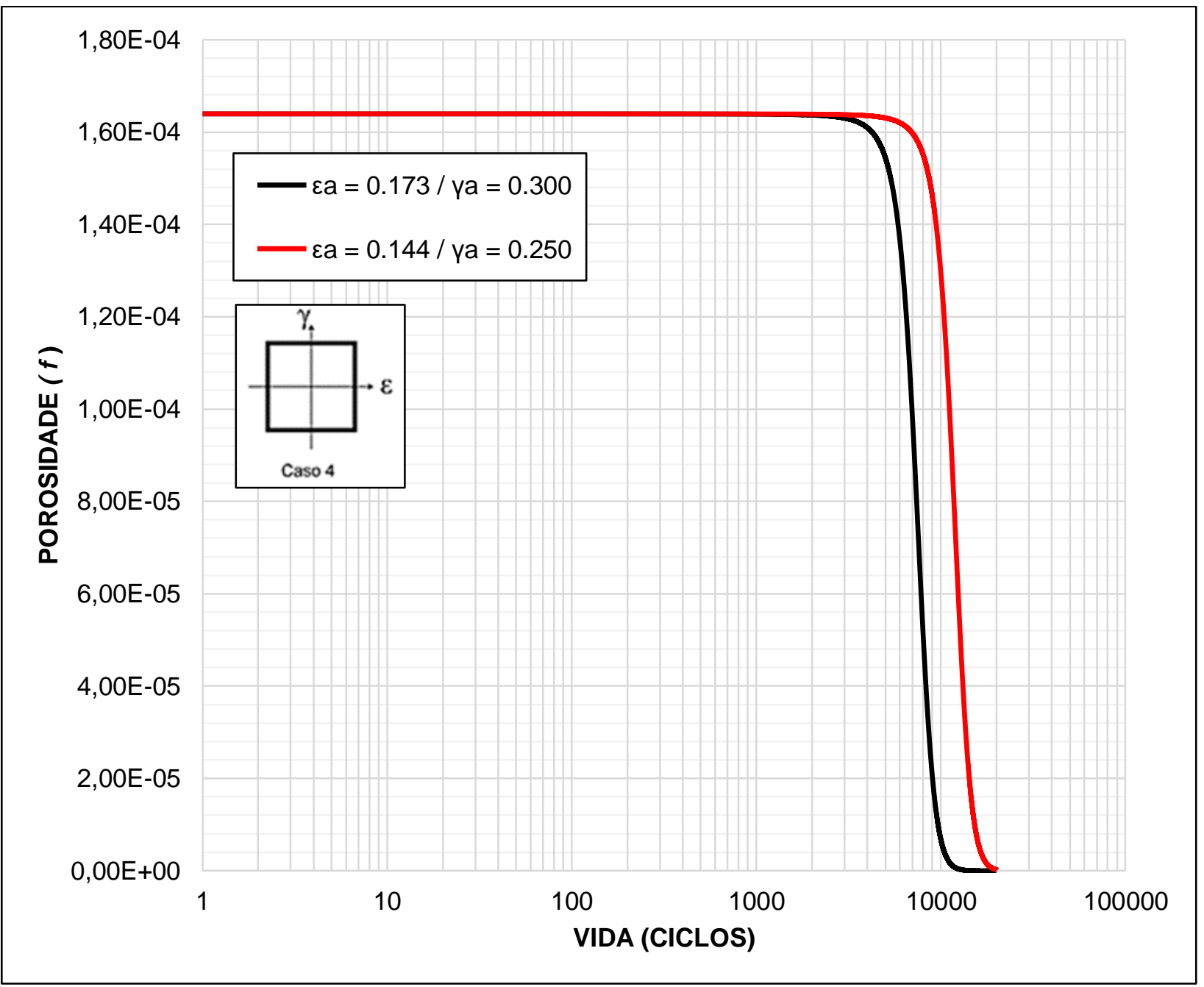

Figura 29. Evolução da porosidade em função do ciclo de vida do Aço S460N. 
Tabela 16. Análise comparativa da vida numérica para vida experimental em trajetória multiaxial não proporcional. Alumínio 6061-T6 (Itoh, 2001).

\begin{tabular}{|c|c|c|c|c|}
\hline \multicolumn{5}{|c|}{ Alumínio 6061-T6 (Itoh, 2001) } \\
\hline Caso & $\varepsilon_{\boldsymbol{a}}$ & $\gamma_{\boldsymbol{a}}$ & $\boldsymbol{N}_{\boldsymbol{E X P}}$ & $\boldsymbol{N}_{\text {NUM }}$ \\
\hline 3 & 0,400 & 0,490 & 1650 & Infinita \\
\hline
\end{tabular}

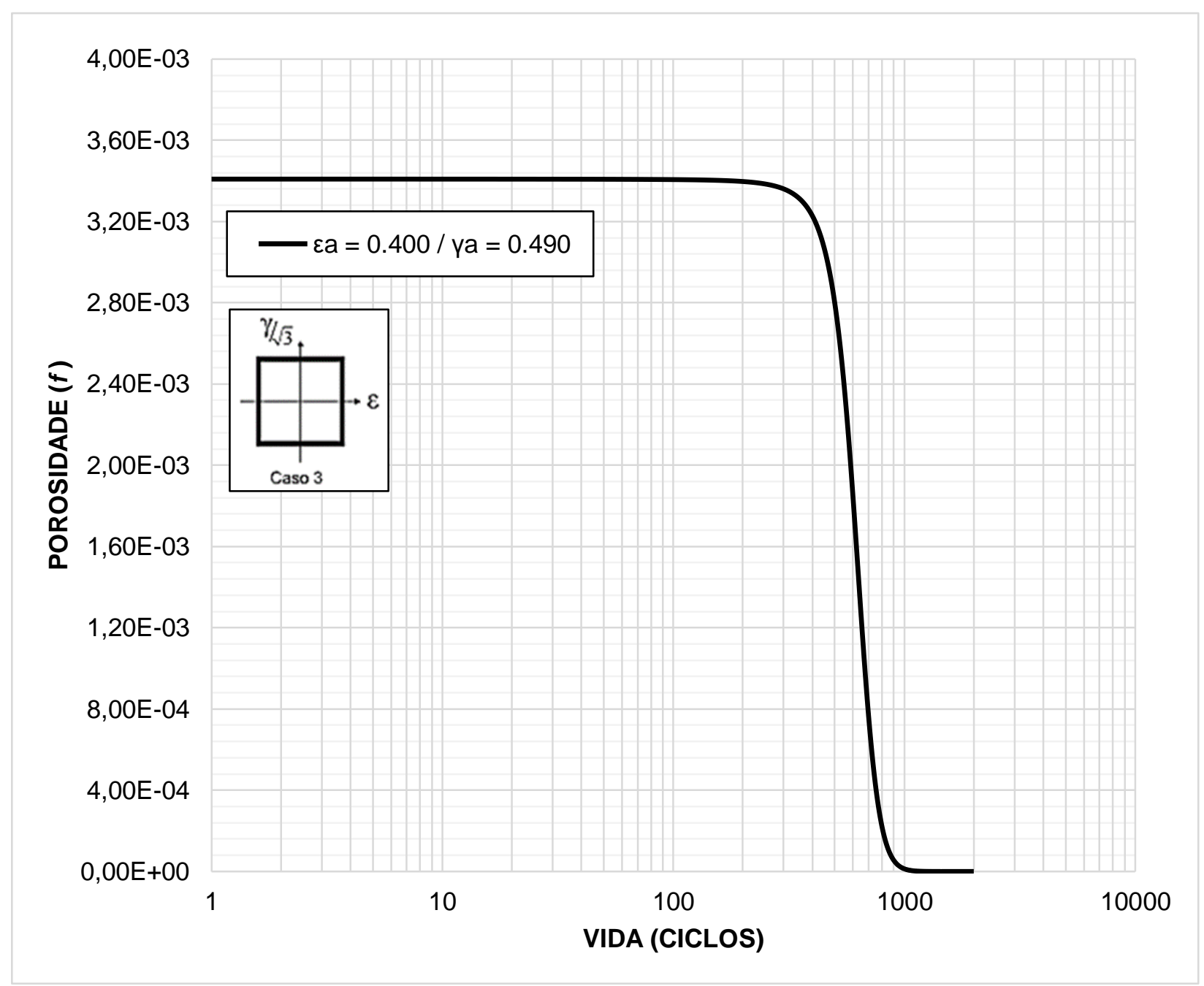

Figura 30. Evolução da porosidade em função do ciclo de vida do Alumínio 6061-T6.

Observa-se, nos gráficos de evolução expostos na Figura 28, Figura 29 e Figura 30, que para a uma trajetória multiaxial não proporcional o modelo de Gurson (1977) não apresentou boa convergência para exatidão satisfatória na previsão de vida a fadiga dos materiais em estudo, dos quais apenas para o aço 304 (Figura 28) notou-se a evolução da porosidade inicial, mesmo que ainda para valores bem inferiores ao da porosidade crítica do material. 
A Figura 31, 32 e 33 apresenta um gráfico da comparação entre a vida em fadiga obtida experimentalmente e a vida estimada pelo modelo de Gurson (1977) para o aço 304.

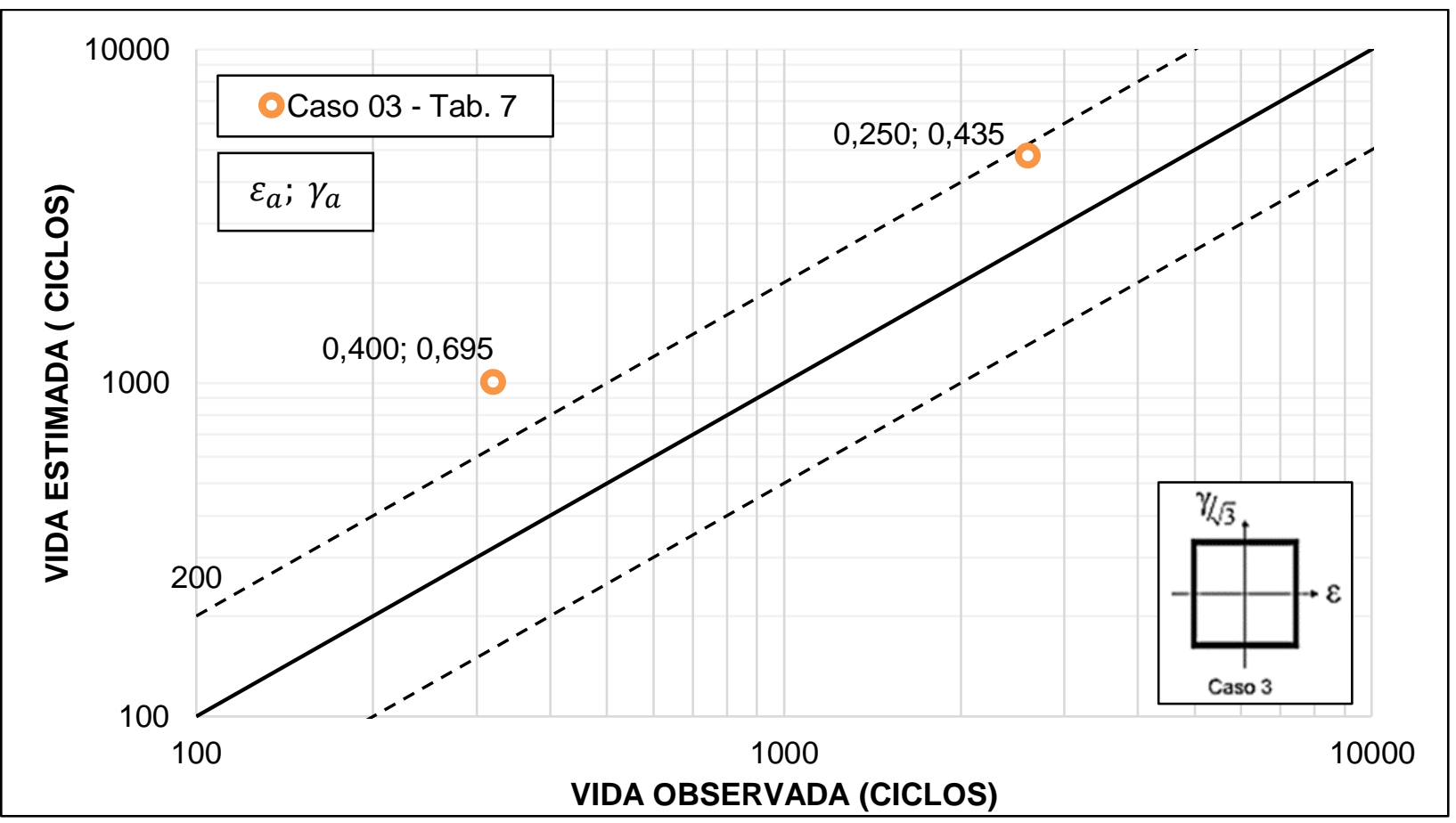

Figura 31. Comparação entre a vida observada experimentalmente e a vida estimada numericamente pelo modelo de Gurson. Aço 304 em trajetória multiaxial não proporcional.

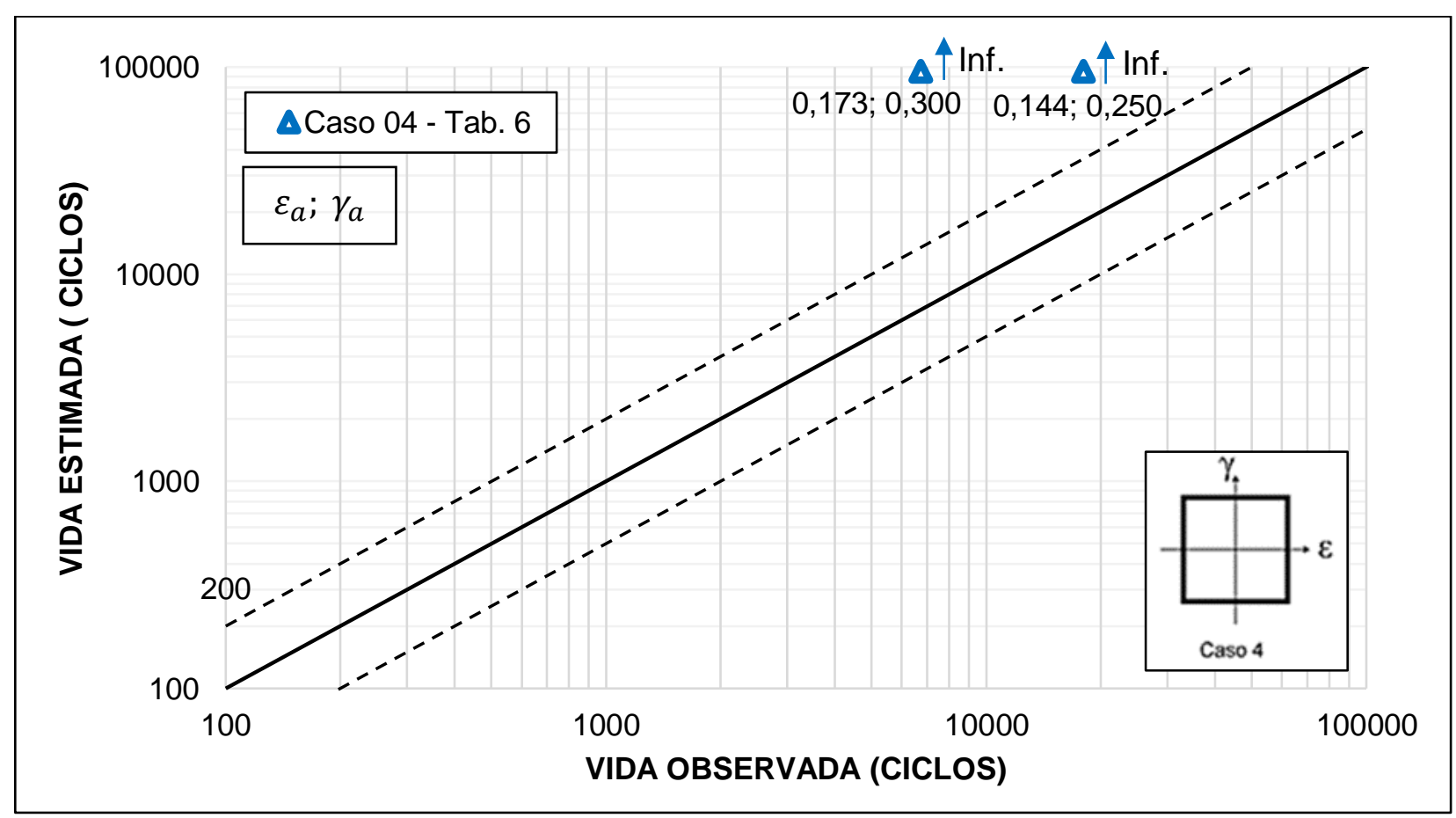

Figura 32. Comparação entre a vida observada experimentalmente e a vida estimada numericamente pelo modelo de Gurson. Aço S460N em trajetória multiaxial não proporcional. 


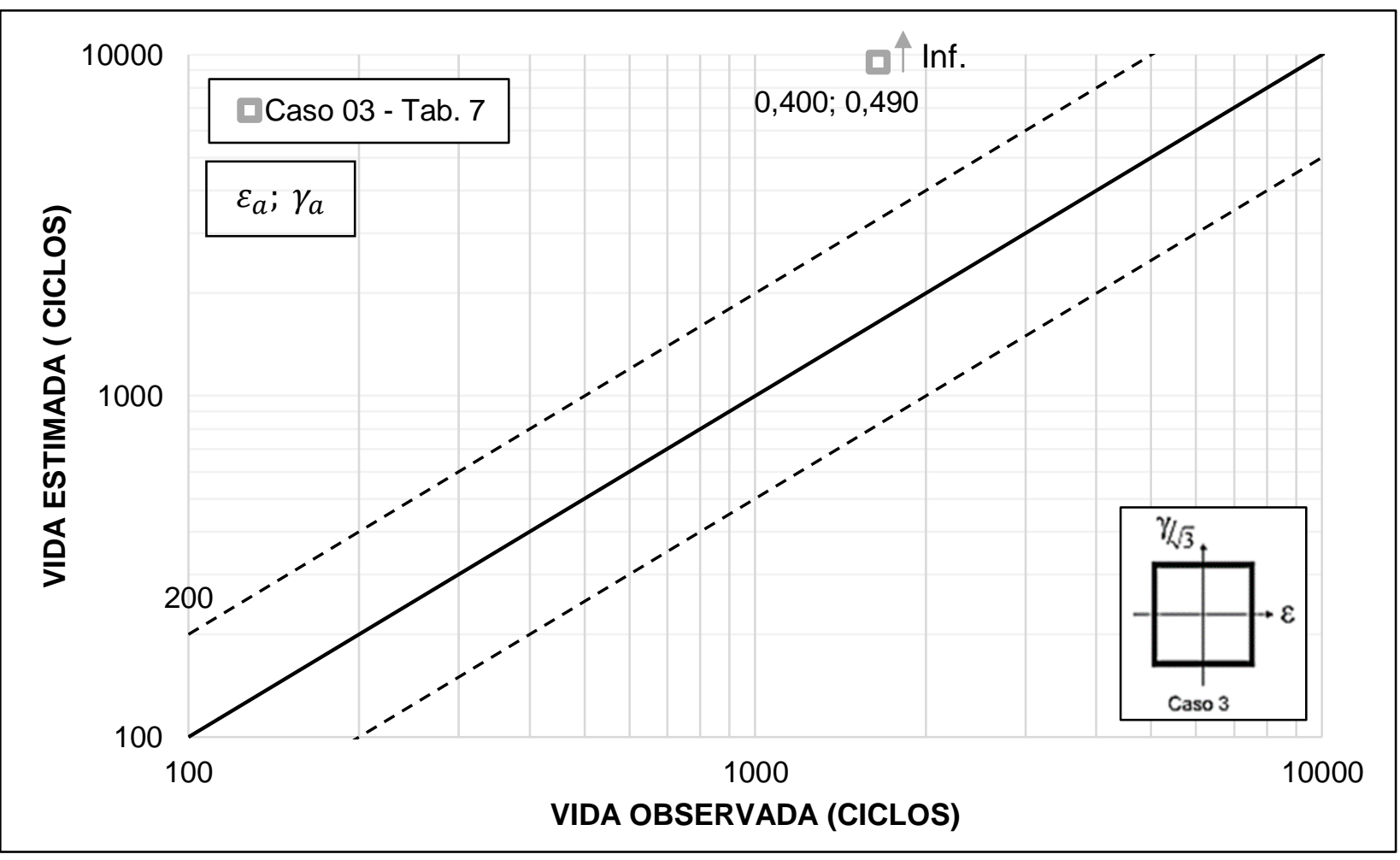

Figura 33. Comparação entre a vida observada experimentalmente e a vida estimada numericamente pelo modelo de Gurson. Alumínio 6061-T6 em trajetória multiaxial não proporcional.

Ao contrário do ocorrido em outras trajetórias neste trabalho simuladas, para os casos multiaxiais não proporcionais o modelo de Gurson (1977) não se mostrou conservador, apresentando valores de vida bem superiores aos observados experimentalmente, para o caso do aço 304, e tendendo a vida infinita para os casos do aço $S 460 \mathrm{~N}$ e do alumínio 6061-T6, o que é inconsistente do ponto de vista físico. Mais uma vez, a ausência de um mecanismo de captura dos efeitos de distorção se mostrou crítico para a previsão de trajetórias que englobem carregamentos cisalhantes.

Outra ocorrência deste ensaio numérico foi que, para o aço S460N e alumínio 6061-T6, durante cada ciclo da trajetória houve o fenômeno de fechamento dos vazios, o que pode ser observado na Figura 29 e Figura 30. Para um melhor entendimento deste efeito, as Figura 35 e Figura 34 (aço 304); Figura 36 e Figura 37 (aço S460N); e Figura 38 (alumínio 6061-T6) ilustram o comportamento de evolução do vazio para 1 (um) ciclo do carregamento aplicado. 


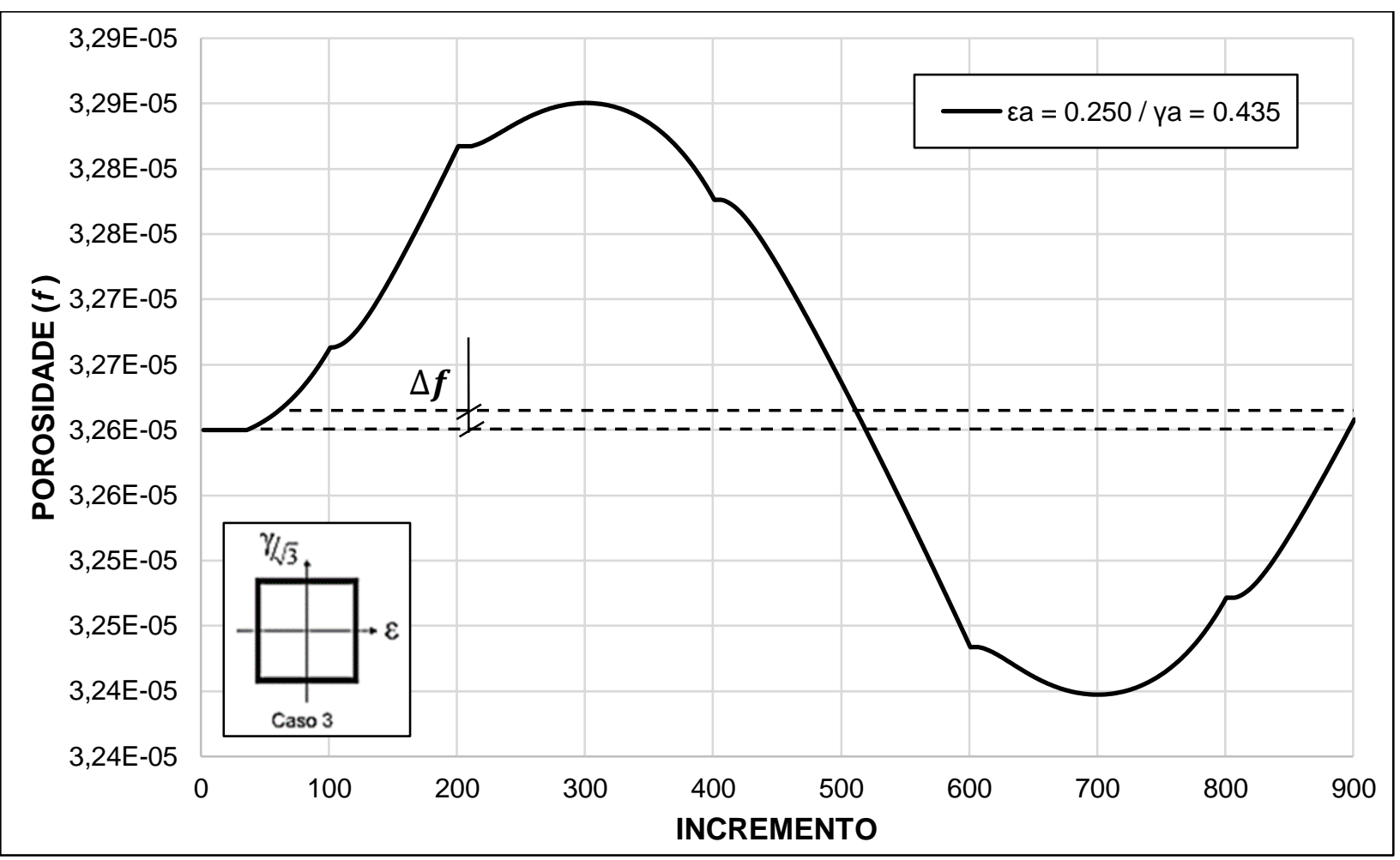

Figura 35. Evolução da porosidade no primeiro ciclo. Aço 304 em trajetória multiaxial - Caso 3 / Tab. 7.

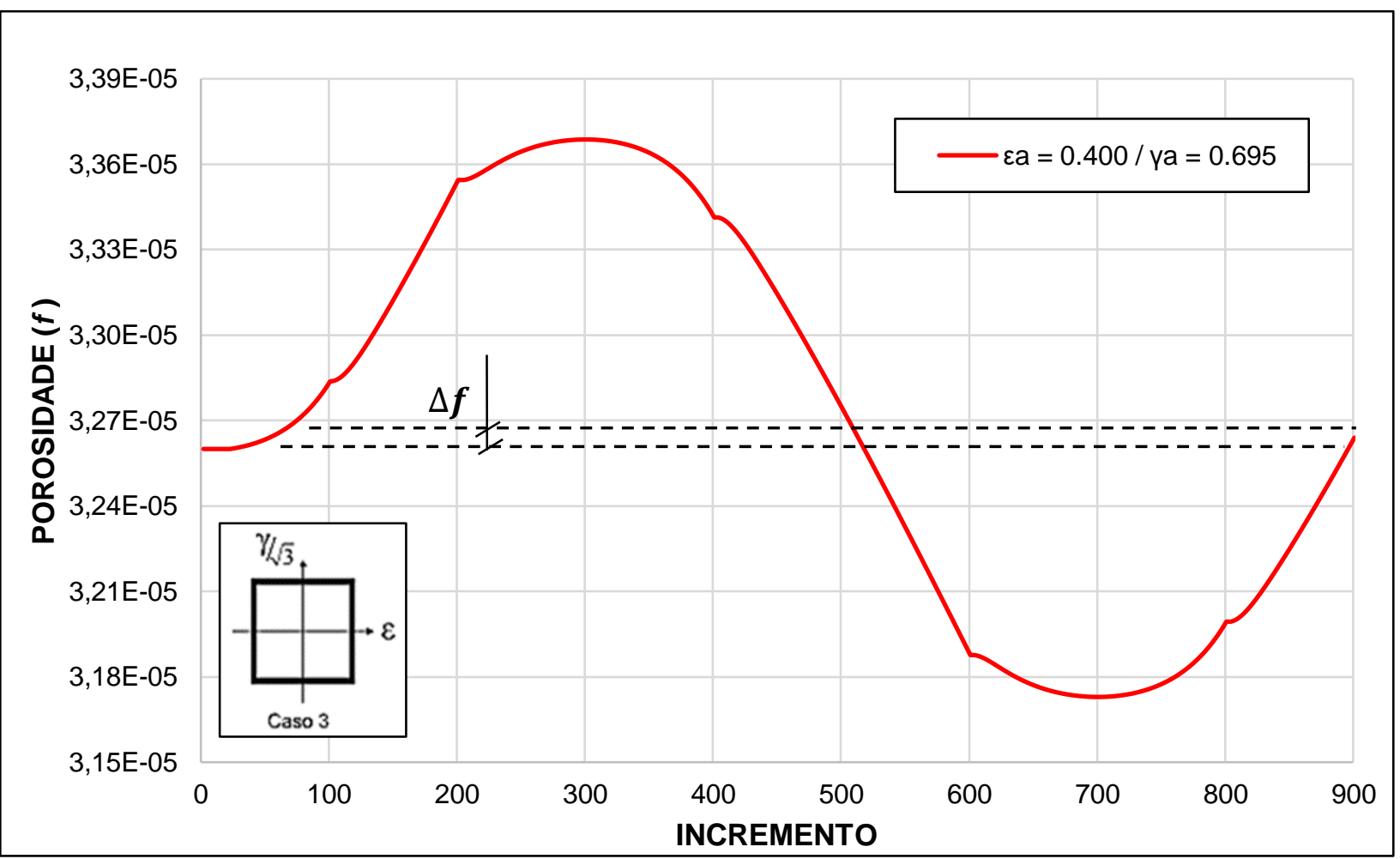

Figura 34. Evolução da porosidade no primeiro ciclo. Aço 304 em trajetória multiaxial - Caso 3 / Tab. 7. 


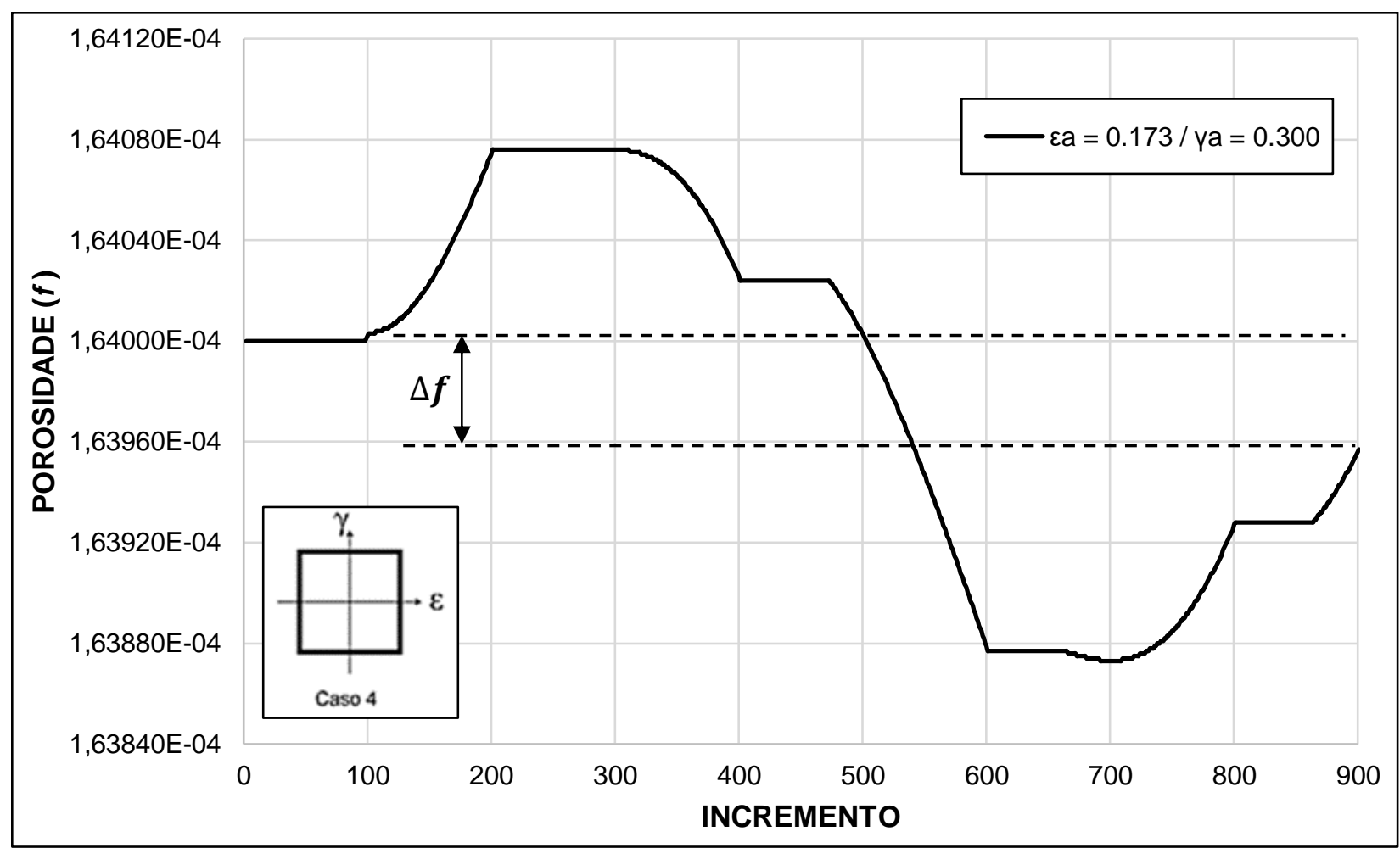

Figura 36. Evolução da porosidade no primeiro ciclo. Aço $S 460 \mathrm{~N}$ em trajetória multiaxial - Caso 4 / Tab. 6.

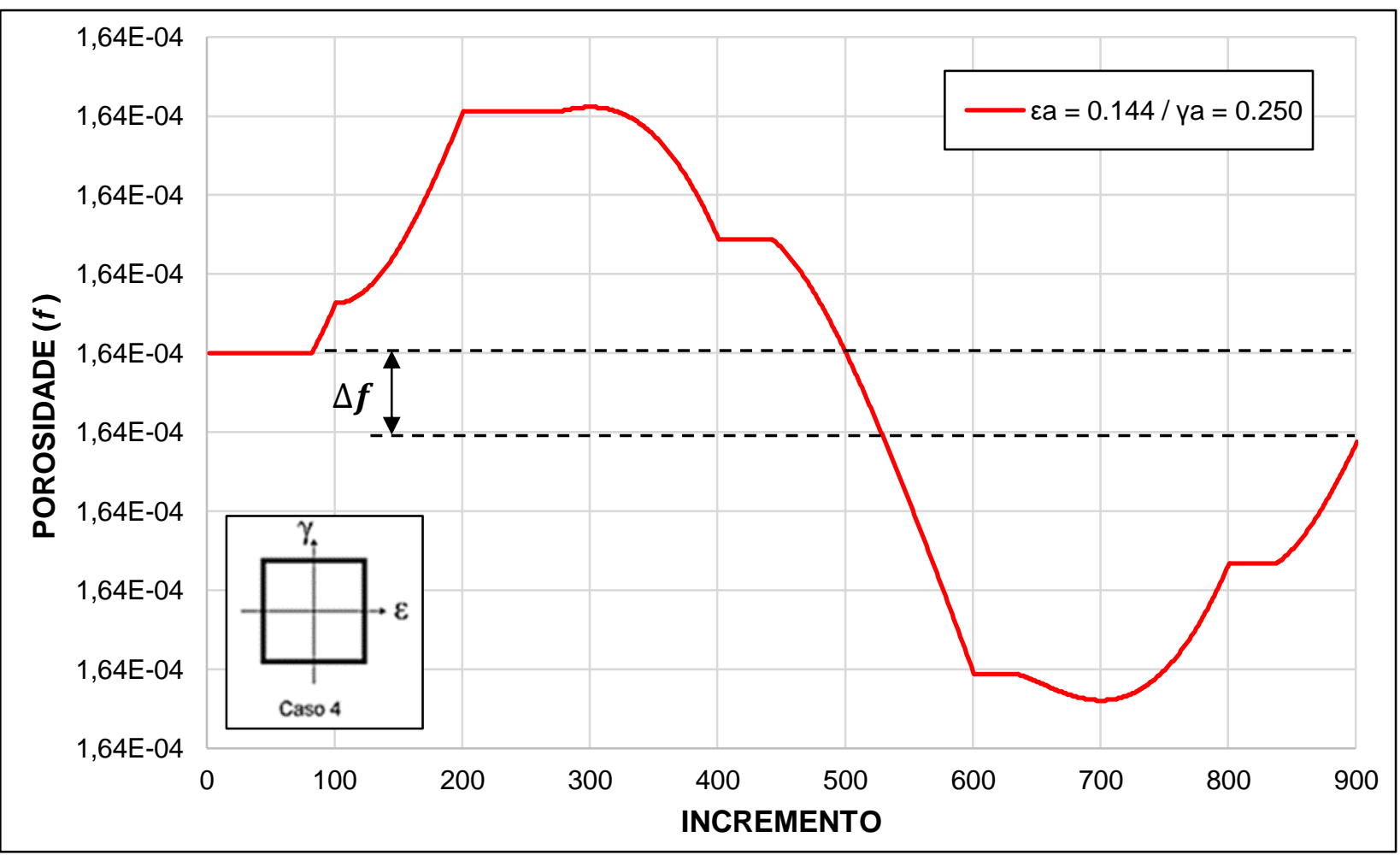

Figura 37. Evolução da porosidade no primeiro ciclo. Aço S460N em trajetória multiaxial - Caso 4 / Tab. 6. 


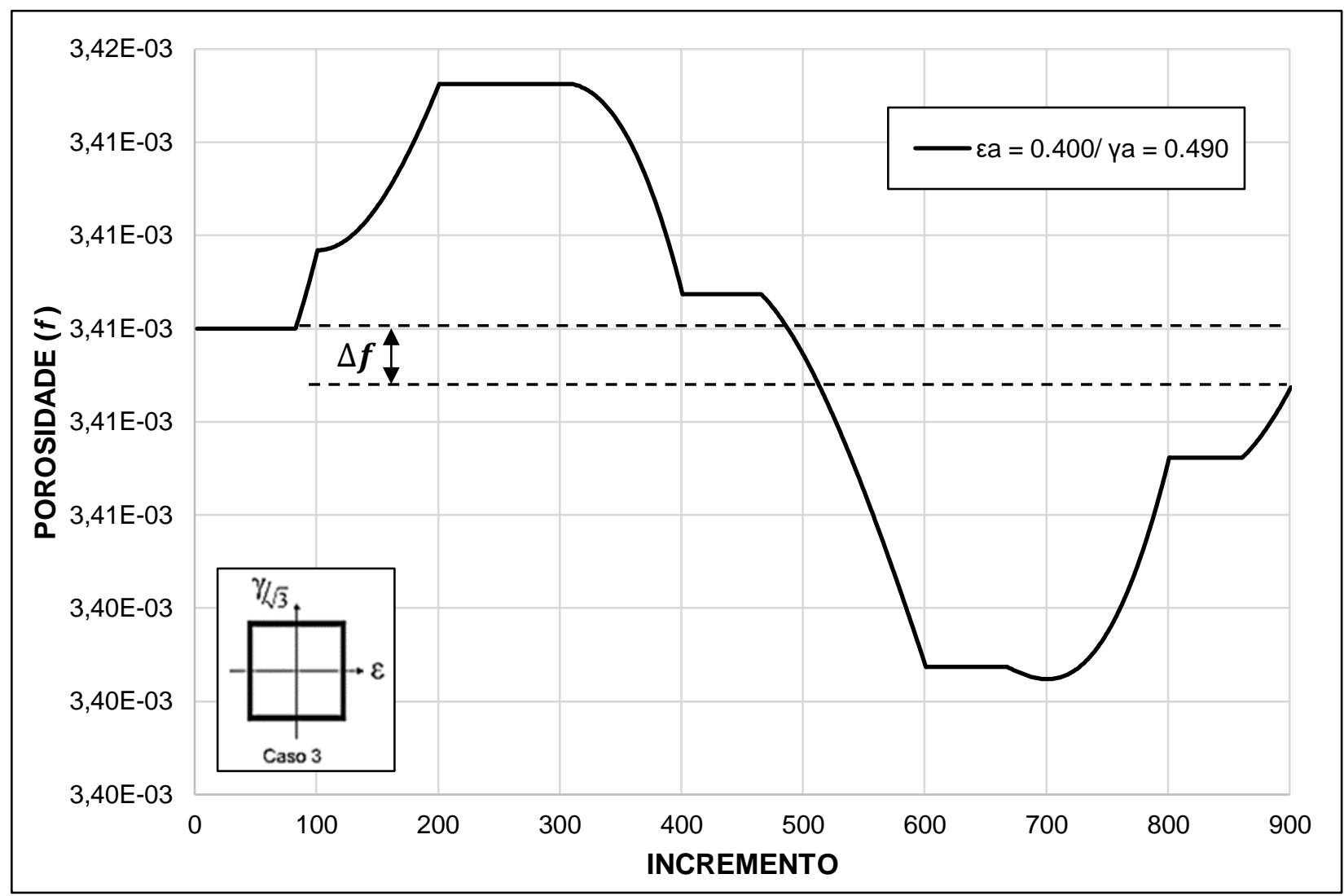

Figura 38. Evolução da porosidade no primeiro ciclo. Alumínio 6061 em trajetória multiaxial - Caso 3 / Tab. 7.

A análise da evolução da porosidade em um ciclo da trajetória multiaxial não proporcional aplicada, ocorre de acordo com o crescimento dos incrementos do ciclo, sendo estes interpretados conforme a Figura 39.

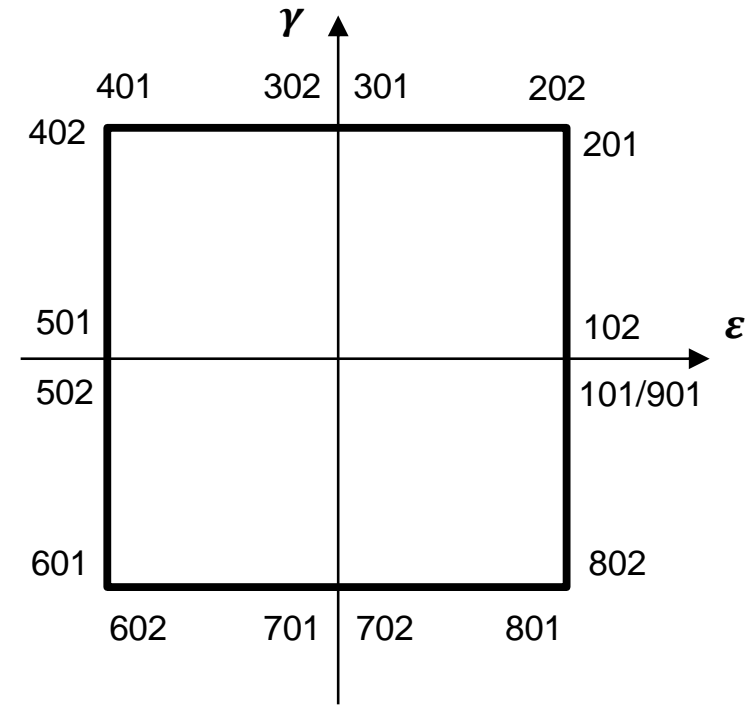

Figura 39. Mapeamento da evolução de incrementos dentro da trajetória multiaxial não proporcional aplicada. 
Desta forma, é possível interpretar que as faixas que contém componentes cisalhantes (102-201; 402-601; e 802-901), são as que mais apresentam perda de precisão por conta dos efeitos de distorção não considerados no modelo em uso.

Nota-se ainda que para o aço 304 (Figura 36), a porosidade ao final do ciclo é, mesmo que em baixa escala, superior a porosidade inicialmente considerada. Este fato gera um aumento da porosidade inicial ciclo a ciclo, entretanto, este pequeno crescimento do vazio não é suficiente para que o valor da porosidade crítica seja alcançado.

Já para o aço S460N e o alumínio 6061-T6 (Figura 37 e Figura 38), a porosidade ao final do ciclo é menor do que a porosidade inicial, ou seja, ciclo a ciclo ocorre o fechamento dos vazios até que estes tendam a zera e a vida seja apresentada como infinita.

Por fim, a Figura 40 apresenta uma visão geral entre todas as comparações realizadas entre vidas observadas experimentalmente e vidas estimadas numericamente pelo modelo de Gurson (1977) expostas neste capítulo.

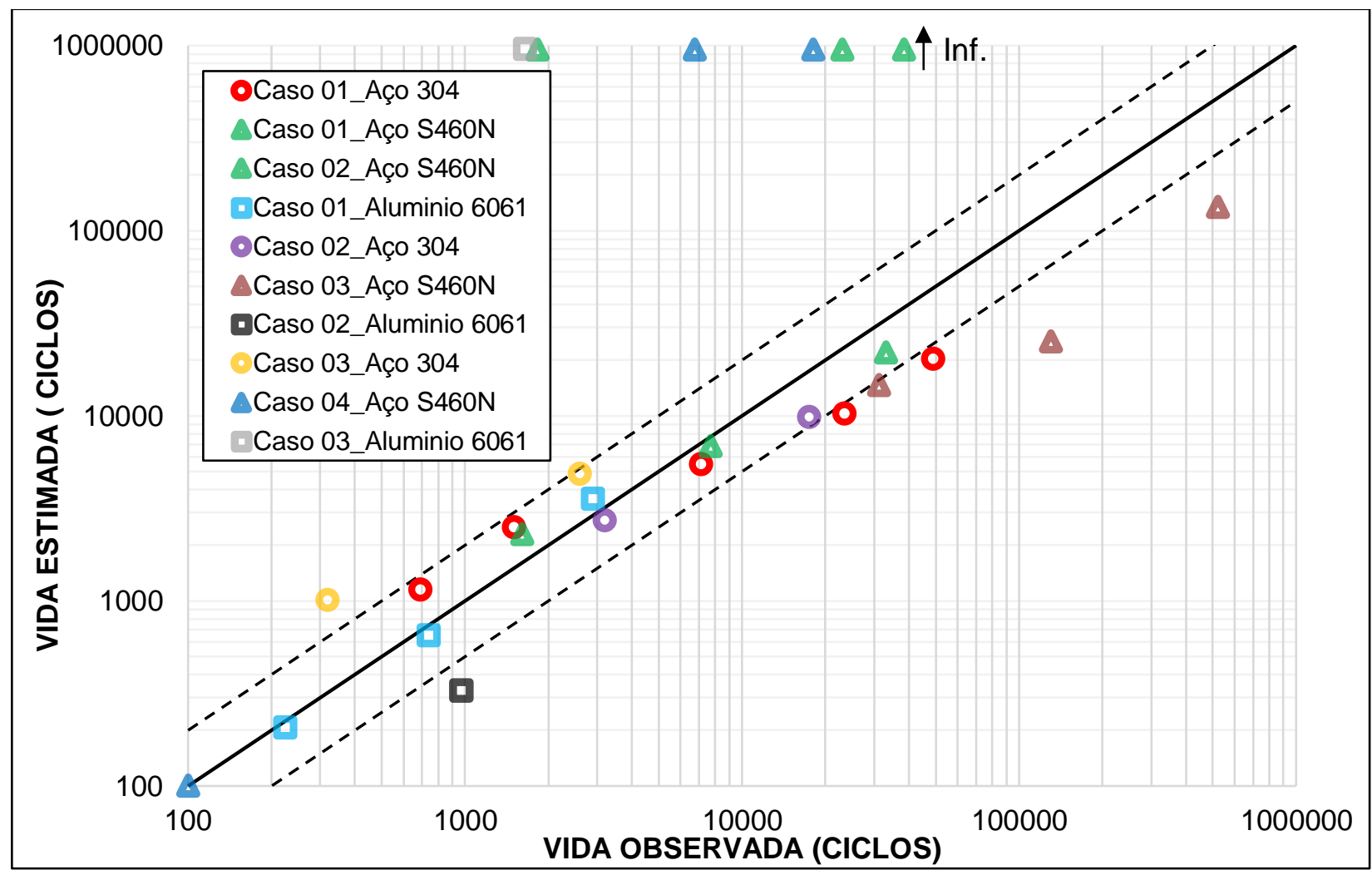

Figura 40. Visão geral da comparação entre a vida observada experimentalmente e a vida estimada numericamente pelo modelo de Gurson para todos os materiais e trajetórias propostas. 


\section{CONCLUSÕES E TRABALHO FUTUROS}

Conforme previsto nos objetivos inicialmente estabelecidos, o presente trabalho consolidou o desenvolvimento do modelo matemático formulado por Gurson (1977) em conjunto com a lei endurecimento cinemático de Armstrong \& Frederick (1966), utilizando para tal a criação de uma rotina implícita de integração numérica capaz de atualizar as variáveis internas estabelecidas pelo problema. Essa rotina, embasada na micromecânica de defeitos, permitiu empregar esta abordagem como uma ferramenta de determinação da vida de materiais submetidos a fadiga.

Para o uso do modelo aplicado a este fim, foi necessária a calibração para os parâmetros materiais ajustados à cada caso de interesse, os quais foram obtidos com base na relação de Ramberg-Osgood (1943).

Assim, a utilização do modelo se completou com o emprego de uma metodologia inversa de identificação paramétrica para definição da porosidade inicial $f_{o}$, a qual é associada a evolução do dano ou a fração volumétrica de vazios, permitindo que vida experimental observada pudesse ser determinada numericamente através um valor de porosidade crítica definida e do uso do modelo para previsão de vida em diferentes trajetórias uniaxiais, multiaxiais proporcionais e multiaxiais não proporcionais, conforme discutido no Cap. 4, onde foi possível observar e concluir que o modelo de Gurson (1977) apresenta boa convergência quando aplicado a valores elevados de amplitude de deformação, casos onde a porosidade atinge o seu valor crítico para uma vida próxima da vida observada experimentalmente.

Já para amplitudes menores de deformação, onde o nível de deformação plástica macroscópica é baixo, o valor de porosidade crítica só é atingido para níveis elevados de vida calculada, estando distante dos valores experimentalmente observados. Para alguns casos, observou-se que a evolução da porosidade não atinge o valor crítico.

Uma importante característica do modelo original de Gurson (1977) constatada é que este não inclui os efeitos de distorção do vazio e, portanto, não permitiu a evolução da porosidade através de carregamentos predominantemente cisalhantes, o que gerou 
falhas na convergência dos resultados numéricos obtidos para as trajetórias que continham este tipo de componente.

Para os casos de carregamento multiaxial não proporcional, onde as componentes cisalhantes apresentavam amplitudes maiores do que as normais, concluiu-se, para o aço 304, um resultado simllar ao identificado para as trajetórias multiaxiais proporcionais. Contudo, para o aço $\$ 460 \mathrm{~N}$ e para o alumínio 6061 -T6, foi constatada a previsão de fechamento dos vazios, o que se mostra como uma característica do material presente para a trajetória aplicada desde o primeiro ciclo de análise.

Por fim, conclui-se que os objetivos traçados puderam ser alcançados com êxito, tendo o presente trabalhado resultado em uma ferramenta capaz de prever, com boa aproximação, a vida a fadiga através de um modelo da micromecânica de defeitos, principalmente, para casos de trajetórias uniaxiais e com trajetórias de contemplem componentes normais de deformação.

Como trabalhos futuros, sugere-se que seja introduzido, ao modelo aqui definido, a capacidade de previsão dos mecanismos de cisalhamento e distorção, o que pode ser executado através de modelos propostos por Nahshon \& Hutchinson (2008), Barsoum \& Faleskog (2007) e Xue (2008). (Malcher, 2012) 


\section{REFERÊNCIA BIBLIOGRAFICA}

Armstrong, P. J., Frederick, C. O. (1966). A mathematical representation of the multiaxial Bauschinger effect. Report RD/B/N731, CEGB, Central Electricity Generating Board, Berkley, UK.

A. L. Gurson. (1977). Continuum Theory of Ductile Rupture by Void Nucleation and Growth: Part I- Yield criteria and Flow Rules for Porous Ductile Media. Journal of Engineering Materials and Technology, 99(76), 2-15. doi:10.1115/1.3443401

Aoyama, N. S. I., Malcher, L., Sahadi, J. V., \& Neves, R. S. (2011). Determination Of The Fracture Initiation In Ductile Material, Based On Gurson's Model Coupled With Shear Mechanisms. $21^{\circ}$ Brazilian Congress of Mechanical Engineering COBEM.

Bai, Y., \& Wierzbicki, T. (2008). A new model of metal plasticity and fracture with pressure and Lode dependence. International Journal of Plasticity, 24(6), 10711096. doi:10.1016/j.ijplas.2007.09.004

Brunig, M., \& Gerke, S. (2011). Simulation of damage evolution in ductile metals undergoing dynamic loading conditions. International Journal of Plasticity, 27(10), 1598-1617. doi:10.1016/j.jplas.2011.02.003

Castro, D. B. V. (2012). Estudo do comportamento em Fadiga do Aço ASTM A297 Gr HP modificado com Nióbio em altas temperaturas, 140.

Chen, B., Peng, X., Fan, J., \& Chen, S. (2005). A constitutive description for casting aluminum alloy A104 based on the analysis of cylindrical and spherical void models. International Journal of Plasticity, 21(11), 2232-2253. doi:10.1016/j.jplas.2005.04.004

Chen, B., Peng, X., Fan, J., \& Chen, S. (2006). An elastoplastic constitutive description based on an ellipsoidal void model. Materials Science and Engineering A, 423(1-2), 230-236. doi:10.1016/j.msea.2005.12.039 
Chimisso, F. E. G., Pacheco, P. M. C. L., \& Maltos, H. C. (1993). Considerações sobre a Previsão de Vida Usando a Mecânica do Dano Contínuo. Pontífica Universidade Católica Do Rio de Janeiro. doi:10.13140/2.1.2526.0804

Cunha, M. A. de C. (2013). Análise Numérica De Modelos De Dano Baseados Na Micromecânica De Defeitos. Journal of Chemical Information and Modeling, 53(9), 1689-1699. doi:10.1017/CBO9781107415324.004

Dahlberg, M., \& Segle, P. (2010). Evaluation Of Models For Cyclic Plasticity Deformation - A Literature Study, 62.

Fei, H., Yazzie, K., Chawla, N., \& Jiang, H. (2012). Modeling fracture of sn-rich (PbFree) solder joints under mechanical shock conditions. Journal of Electronic Materials, 41(8), 2089-2099. doi:10.1007/s11664-012-2079-5

Fei, H., Yazzie, K., Chawla, N., \& Jiang, H. (2012). The effect of random voids in the modified Gurson model. Journal of Electronic Materials, 41(2), 177-183. doi:10.1007/s11664-011-1816-5

Fesich, T. M., Mohan, P., \& Marzougui, D. (2008). A Study of the Gurson Damage Model and Numerical Simulation of Ductile Failure in LS-DYNA. LS-DYNA Anwenderforum, Bamberg 2008, 1-12.

G. Z., V., \& Kiousis, P. D. (1985). Stress Rate And The Lagrangian Formulation Of The Finite-Strain Plasticity For A Von Mises Kinematic Hardening Model, 23(12 December), 95-107.

Neves, R. S. (2015). Constitutivos Para Plasticidade Cíclica Faculdade Gama / Faculdade De Tecnologia Programa De Pós-Graduação Em Integridade De.

Gates, R. L. (2013). A Finite Element Implementation of a Ductile Damage Model for Small Strains, (December), 69. Retrieved from http://arxiv.org/abs/1302.2439

Hancell, P. J., \& Harvey, S. J. (1979). The Use Of Kinematic Hardening Models In Multi-Axial Cyclic Plasticity, I, 271-279. 
Heeres, O. M. (2001). Modern Strategies for the Numerical Modeling of the Cyclic and Transient Behavior of Soils. Delft University of Technology.

Jhonatan da Ponte Lopes. (2014). Implementação Implícita Do Modelo De Plasticidade Cíclica De Chaboche. Universidade de Brasilia.

Jiang, W., Li, Y., Shu, Y., \& Fan, Z. (2013). Analysis of Metallic Ductile Fracture by extended Gurson models. 13th International Conference on Fracture, (1), 16-21.

Jiang, Y., \& Sehitoglu, H. (1996). Modeling of Cyclic Ratchetting Plasticity, Part I: Development of Constitutive Relations, 63(September), 720-725.

Jiang, Y., \& Sehitoglu, H. (1996). Modeling of Cyclic Ratchetting Plasticity, Part II : Comparison of Model Simulations With Experiments, 63(September).

Jiang, Y., \& Zhang, J. (2008). Benchmark experiments and characteristic cyclic plasticity deformation. International Journal of Plasticity, 24(9), 1481-1515. doi:10.1016/j.ijplas.2007.10.003

Kattan, P. I., \& Voyiadjis, G. Z. (1990). A coupled theory of damage mechanics and finite strain elasto-plasticity I. Damage and elastic deformations. International Journal of Engineering Science, 28(5), 421-435.

Kossa, A., \& Szabó, L. (2009). Exact integration of the von Mises elastoplasticity model with combined linear isotropic-kinematic hardening. International Journal of Plasticity, 25(6), 1083-1106. doi:10.1016/j.ijplas.2008.08.003

Krol, O. (2007). Thermo-Mechanical Modelling of Solids and Interfaces - Theory, Numerics and Applications -. Interfaces.

Lestriez, P., Bogard, F., Shan, J. L., \& Guo, Y. Q. (2007). Damage Evolution on Mechanical Parts Under Cyclic Loading, 1389-1395.

Lopes, J. da P. (2014). Implementação Implícita Do Modelo De Plasticidade Cíclica De Chaboche. Universidade de Brasília. 
Luo, C., Wei, J., Chattopadhyay, A., \& Jiang, H. (2007). A Void Growth And A Cyclic Model In Ductile Material Using Mechanism-Based Strain Gradient Crystal Plasticity Theory. Asme International, (November), 11-15.

Malcher, L. (2012). Continuum Modelling and Numerical Simulation of Damage for Ductile Materials.

Malcher, L. (2011). DA MECÂNICA DO DANO CONTÍNUO: Uma evolução do modelo de Lemaitre para redução da dependência do ponto de calibração. Universidade de Brasilia.

Metzger, M., \& Seifert, T. (2013). On the exploitation of Armstrong-Frederik type nonlinear kinematic hardening in the numerical integration and finite-element implementation of pressure dependent plasticity models. Computational Mechanics, 52(3), 515-524. doi:10.1007/s00466-012-0828-1

Nascimento, J. L. do, Silva, A. A., \& Barbosa, J. M. A. (2005). Simulação Computacional Do Dano Em Um Modelo De Material Elastoplástico Sob Carregamento Cíclico, (3), 1-6.

Needleman, A., Tvergaard, V., \& Hutchinson, J. W. (1992). Void Growth in Plastic Solids. Topics in Fracture and Fatigue. doi:10.1007/978-1-4612-2934-6_4

Neves, R. S. (2015). Implementação E Validação De Modelos Constitutivos Para Plasticidade Cíclica. Universidade de Brasília.

Paul, S. K., Sivaprasad, S., Dhar, S., Tarafder, M., \& Tarafder, S. (2010). Simulation of cyclic plastic deformation response in SA333 C-Mn steel by a kinematic hardening model. Computational Materials Science, 48(3), 662-671. doi:10.1016/j.commatsci.2010.02.037

Pires, F. M. A. (2001). Modelação por Elementos Finitos da Iniciação da Fractura Dúctil nos Processos de Enformação Plástica em Massa. 
Puzrin, A. M., \& Houlsby, G. T. (2001). Fundamentals of kinematic hardening hyperplasticity. International Journal of Solids and Structures, 38(21), 3771-3794. doi:10.1016/S0020-7683(00)00238-9

Ramaswamy, S., \& Aravas, N. (1998). Finite element implementation of gradient plasticity models Part I: Gradient-dependent yield functions. Computer Methods in Applied Mechanics and Engineering, 163(98), 11-32. doi:10.1016/S00457825(98)00028-0

Rossi, R. (2005). Proposições aplicações considerando o método de Galerkin livre de elementos. Thesis. Universidade Federal de Santa Catarina Programa. Retrieved from http://repositorio.ufsc.br/handle/123456789/102994

Sahadi, J. V. (n.d.). Estudo Da Fratura Dúctil Através De Modelos Dependentes Do Terceiro Invariante Do Tensor Desviador.

Souza Neto, E. de, Peric, D., \& Owen, D. R. J. (2008). Computational Methods for Plasticity. Computational methods for plasticity-theory and applications (Vol. 55). doi:10.1002/9780470694626

Voyiadjis, G. Z., Hoseini, S. H., \& Farrahi, G. H. (2012). Effects of stress invariants and reverse loading on ductile fracture initiation. International Journal of Solids and Structures, 49(13), 1541-1556. doi:10.1016/j.ijsolstr.2012.02.030

Wen, Z. (2012). Modeling Of Ductile Fracture In Steel Structures For Monotonic And Cyclic Loading.

Wu, S., Reis, A., Teixeira, P., Da Rocha, A. B., \& Lino, J. (2012). Damage prediction in incremental forming by using Lemaitre damage model. AIP Conference Proceedings, 1479(1), 1600-1605. doi:10.1063/1.4756471

Zheng, H., Wang, J., Huang, J. Y., Wang, J., \& Mao, S. X. (2014). Void-assisted plasticity in Ag nanowires with a single twin structure. Nanoscale, 6, 9574-9578. http://doi.org/10.1039/C3NR04731H 


\section{ANEXO I - EXPRESSÕES ÚTEIS}

i.

$$
\frac{\partial \boldsymbol{N}_{\boldsymbol{d}_{n+1}}}{\partial \boldsymbol{S}_{n+1}}=\boldsymbol{I}^{4}
$$

ii.

$$
\frac{\partial \boldsymbol{N}_{\boldsymbol{d}_{n+1}}}{\partial \boldsymbol{\beta}_{n+1}}=-\boldsymbol{I}^{4}
$$

iii.

$$
\frac{\partial N_{v_{n+1}}}{\partial p_{n+1}}=\frac{1}{2} f_{n+1} \cosh \left(\frac{3 p_{n+1}}{2 \sigma_{y}}\right)
$$

iv.

$$
\frac{\partial N_{v_{n+1}}}{\partial f_{n+1}}=\frac{1}{3} \sigma_{y} \operatorname{senh}\left(\frac{3 p_{n+1}}{2 \sigma_{y}}\right)
$$

V.

$$
\frac{\partial N_{v_{n+1}}}{\partial \boldsymbol{\beta}_{n+1}}=0
$$

vi.

$$
\frac{\partial \boldsymbol{\beta}_{n+1}}{\partial \boldsymbol{\beta}_{n+1}}=I^{4}
$$

vii.

$$
\begin{aligned}
& \frac{\partial \sqrt{A}}{\partial \boldsymbol{S}_{n+1}}=\frac{1}{2 \sqrt{A}}\left(\frac{2}{3} \cdot 2 \boldsymbol{\eta}\right), \text { onde, substituindo no termo } \frac{\partial \sqrt{A}}{\partial \boldsymbol{S}_{n+1}} \otimes \boldsymbol{\beta}_{n+1}, \text { tem }- \text { se: } \\
& \frac{\partial \sqrt{A}}{\partial \boldsymbol{S}_{n+1}} \otimes \boldsymbol{\beta}_{n+1}=\frac{1}{2 \sqrt{A}} \frac{2}{3} 2 \boldsymbol{\eta} \otimes \boldsymbol{\beta}_{n+1}
\end{aligned}
$$


viii.

$$
\frac{\partial \sqrt{A}}{\partial p_{n+1}}=\frac{1}{2 \sqrt{A}}\left\{\frac{2}{3}\left[f_{n+1}{ }^{2} \sigma_{y} \operatorname{senh}\left(\frac{3 p_{n+1}}{2 \sigma_{y}}\right) \cosh \left(\frac{3 p_{n+1}}{2 \sigma_{y}}\right)\right]\right\}
$$

ix.

$$
\frac{\partial \sqrt{A}}{\partial f_{n+1}}=\frac{1}{2 \sqrt{A}}\left\{\frac{2}{3}\left[\frac{2}{3} f_{n+1} \sigma_{y}^{2} \operatorname{senh}\left(\frac{3 p_{n+1}}{2 \sigma_{y}}\right) \operatorname{senh}\left(\frac{3 p_{n+1}}{2 \sigma_{y}}\right)\right]\right\}
$$

$x$.

$$
\begin{aligned}
& \frac{\partial \sqrt{A}}{\partial \boldsymbol{\beta}_{n+1}}=\frac{1}{2 \sqrt{A}}\left(\frac{2}{3} \cdot-2 \boldsymbol{\eta}\right), \text { onde, substituindo no termo } \frac{\partial \sqrt{A}}{\partial \boldsymbol{\beta}_{n+1}} \otimes \boldsymbol{\beta}_{n+1}, \text { tem }- \text { se: } \\
& \frac{\partial \sqrt{A}}{\partial \boldsymbol{\beta}_{n+1}} \otimes \boldsymbol{\beta}_{n+1}=\frac{1}{2 \sqrt{A}} \frac{2}{3}(-2) \boldsymbol{\eta} \otimes \boldsymbol{\beta}_{n+1}
\end{aligned}
$$




\section{ANEXO II - ALGORITMO DE INTEGRAÇÃO NUMÉRICA}

! BEGIN SUBROUTINE SUAR

! STATE UPDATE PROCEDURE FOR THE ANDRADE \& REIS MODEL FOR 3D PROBLEMS

!

! MALCHER \& BORTOLI, AGOSTO, 2016

SUBROUTINE SUGAF3D(DGAMA, IPROPS , LALGVA, NTYPE , RPROPS , RSTAVA , STRAN , \&

STRES , NRPROP , NIPROP , NRSTAV , NSTRA , NSTRE , NLALGV, IINCS)

IMPLICIT NONE

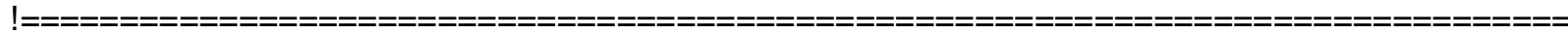

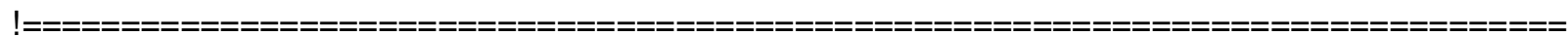

IPARAMETER DECLARATION

INTEGER, PARAMETER:: IPHARD=8, KSTRE=6

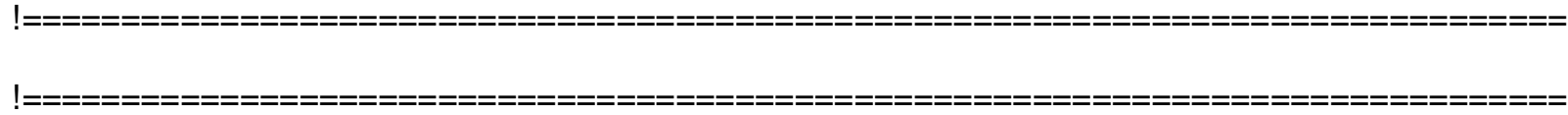

IDATA DECLARATION

$\operatorname{REAL}(8) \mathrm{R} 0 \quad / 0.0 \mathrm{D0} /$

REAL(8) RP5 /0.5D0/

REAL(8) R1 /1.0D0/

REAL(8) R2 /2.0D0/

$\operatorname{REAL(8)~R3\quad /3.0D0/~}$

REAL(8) R4 /4.0D0/

REAL(8) R5 /5.0D0/

REAL(8) R27 /27.0D0/

REAL(8) R81 /81.0D0/

REAL(8) R243 /243.0D0/

REAL(8) R1458 /1458.0D0/

REAL(8) TOL /1.D-06/ 


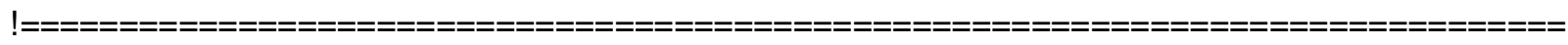

! DECLARATION OF ARGUMENTS

INTEGER NTYPE , NRPROP , NIPROP , NRSTAV , NSTRA, NSTRE , NLALGV, IINCS

REAL(8) DGAMA

INTEGER, DIMENSION(NIPROP) :: IPROPS

REAL(8), DIMENSION(NRPROP) :: RPROPS

REAL(8), DIMENSION(NRSTAV) :: RSTAVA

REAL(8), DIMENSION(NSTRA) :: STRAN

REAL(8), DIMENSION(NSTRE) :: STRES

LOGICAL, DIMENSION(NLALGV) :: LALGVA

! DECLARATION OF LOCAL VARIABLES

LOGICAL IFPLAS, SUFAIL

INTEGER I , J , NHARD , IITER , K

REAL(8) EPBARN, YOUNG , POISS , SIGMAT , SIGMAS, GMODU , BULK , R2G , R3G , \& EEV , P , EEVD3, VARJ2T, QTRIAL, DETS , SIGMAY, XI , PHI , \& EPBAR , HSLOPE , NORMS , SEQ , EQ2 , ADBETA , BDBETA , CDBETA, DDBETA , \& RESNOR, HKSLOPE, BKIN , EQ3, POROSO , POROSN , POROS, PTRIAL, TEMP,VARJ2,ALPHAV, TEMP2, \&

EQ4, DALPHAP, DALPHAF 
REAL(8) PLFUN, DPLFUN

! FOURTH ORDER IDENTITY TENSOR

REAL(8), DIMENSION(NSTRE,NSTRE) :: FOID

! SECOND ORDER IDENTITY TENSOR

REAL(8), DIMENSION(NSTRE) : :: SOID

! DEVIATORIC INDENTITY TENSOR

REAL(8), DIMENSION(NSTRE,NSTRE) :: DFOID

! DEVIATORIC STRAIN TENSOR

REAL(8), DIMENSION(NSTRE) :: EET

REAL(8), DIMENSION(NSTRE) :: STRIAL, BACKN, BACK, ETAN, ETA

REAL(8), DIMENSION(NSTRE) :: SINVT

REAL(8), DIMENSION(NSTRE) :: PROSINVT

REAL(8), DIMENSION(NSTRE) :: BETA

REAL(8), DIMENSION(NSTRE) :: ALPHAD

REAL(8), DIMENSION(NSTRE) ::: EQ1, EQ5

REAL(8), DIMENSION(NSTRE,NSTRE) :: SDOTS

REAL(8), DIMENSION(NSTRE) :: DXI

REAL(8), DIMENSION(NSTRE,NSTRE) :: SITDSIT

REAL(8), DIMENSION(NSTRE,NSTRE) :: PROSITDSIT

REAL(8), DIMENSION(NSTRE,NSTRE) :: SITDS

REAL(8), DIMENSION(NSTRE,NSTRE) :: PROSITDS

REAL(8), DIMENSION(NSTRE,NSTRE) :: DSITDS

REAL(8), DIMENSION(NSTRE,NSTRE) :: PRODSITDS

REAL(8), DIMENSION(NSTRE,NSTRE) :: SDSINVT

REAL(8), DIMENSION(NSTRE,NSTRE) :: DBETA

REAL(8), DIMENSION(NSTRE,NSTRE) :: DALPHA, DALPHAB

REAL(8), DIMENSION(15,15) :: MATRIX 
REAL(8), DIMENSION(15) :: RHS

REAL(8), DIMENSION(15) :: RES

REAL(8), DIMENSION(NSTRE,NSTRE) :: DXIDBETA

! VARIÁVEIS NECESSÁRIAS PARA A DEFINIÇÃO DA VARIÁVEL DE

! ENCRUAMENTO

! FÁBIO REIS \& FILIPE XAVIER - AUGUST, 2012

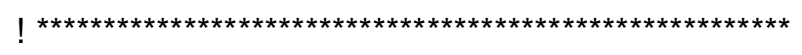

REAL(8), DIMENSION(NSTRE) :: SIGMA

! DUPLA CONTRACÇÃO ENTRE DALPHA E O TENSOR DAS TENSÕES

! GLOBAIS

REAL(8), DIMENSION(NSTRE) :: DC_DALPHA_SIGMA

! DUPLA CONTRACÇÃO ENTRE ALPHA E SIGMA

REAL(8) DC_ALPHA_SIGMA

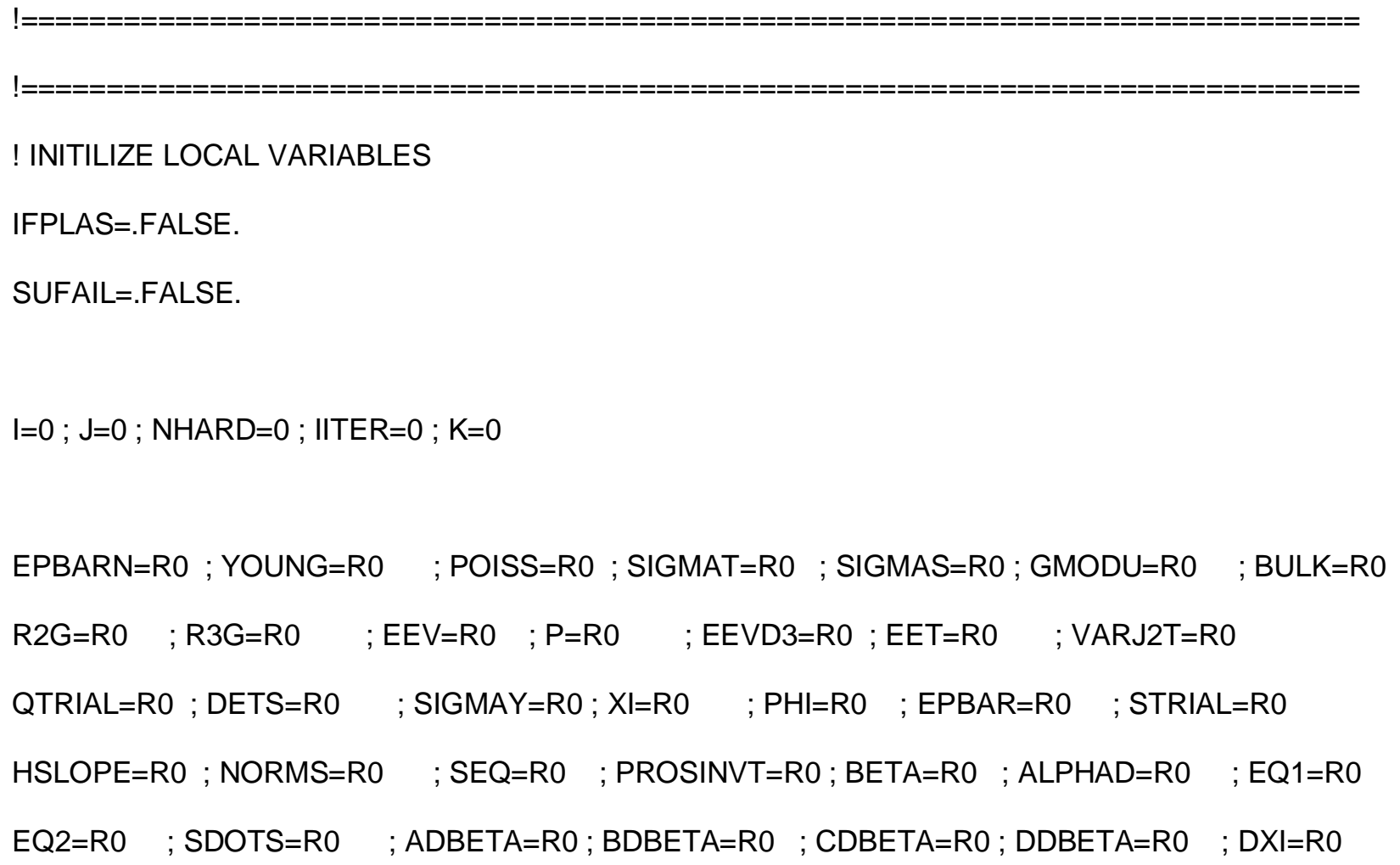




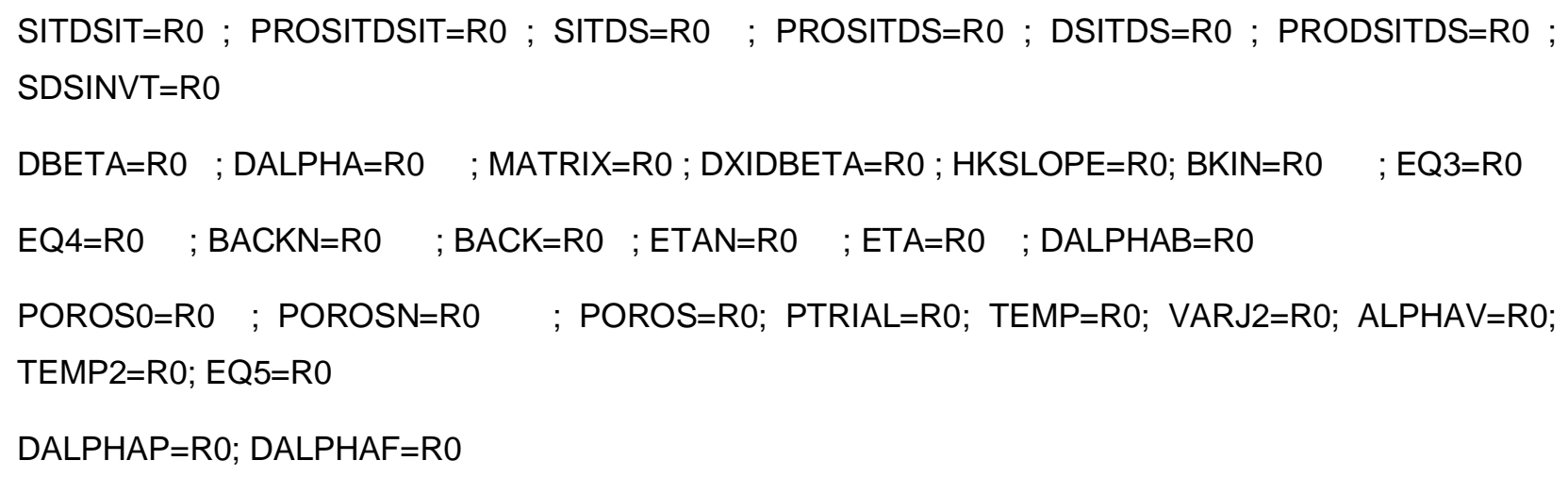


ENDDO

$\operatorname{DFOID}(4,4)=\operatorname{DFOID}(4,4)^{\star} \mathrm{R} 2$

$\operatorname{DFOID}(5,5)=\operatorname{DFOID}(5,5)^{\star} \mathrm{R} 2$

$\operatorname{DFOID}(6,6)=\operatorname{DFOID}(6,6)^{*} \mathrm{R} 2$

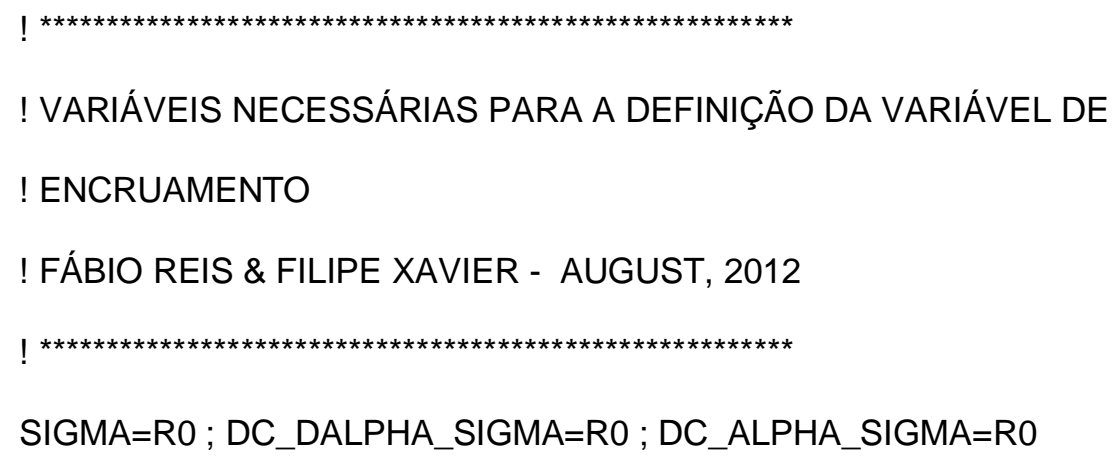




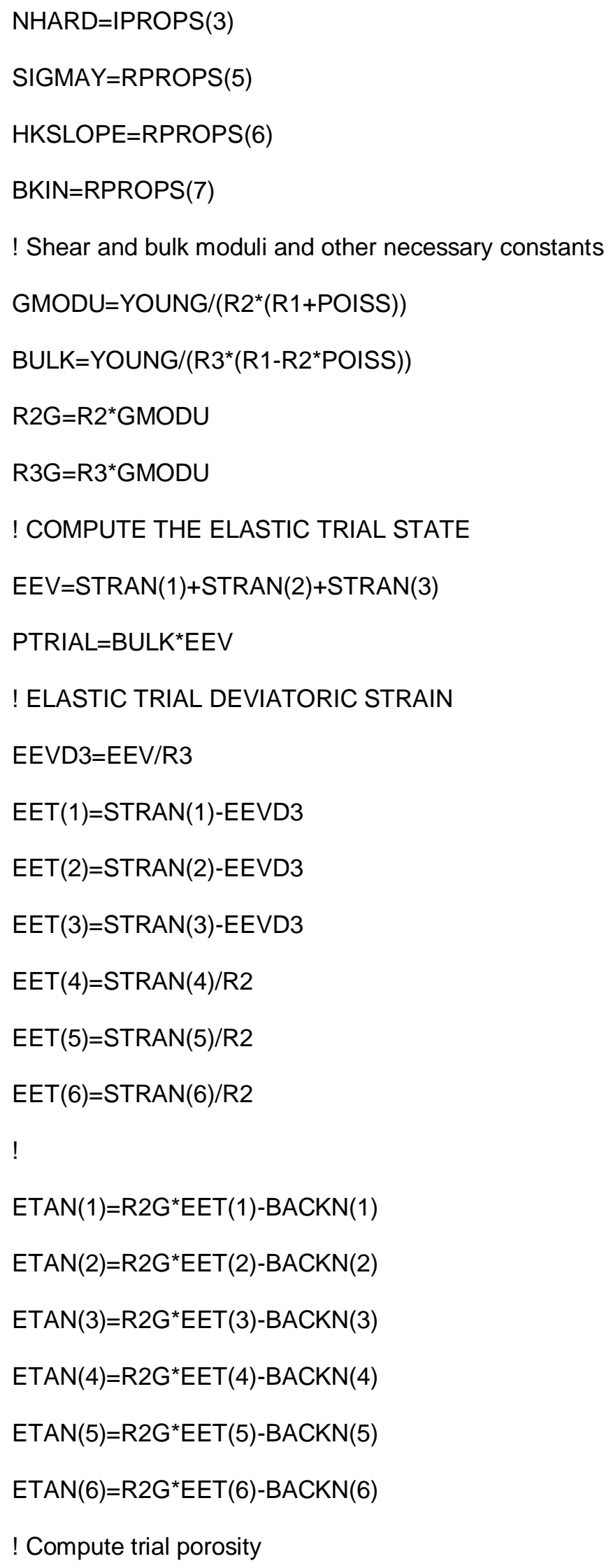




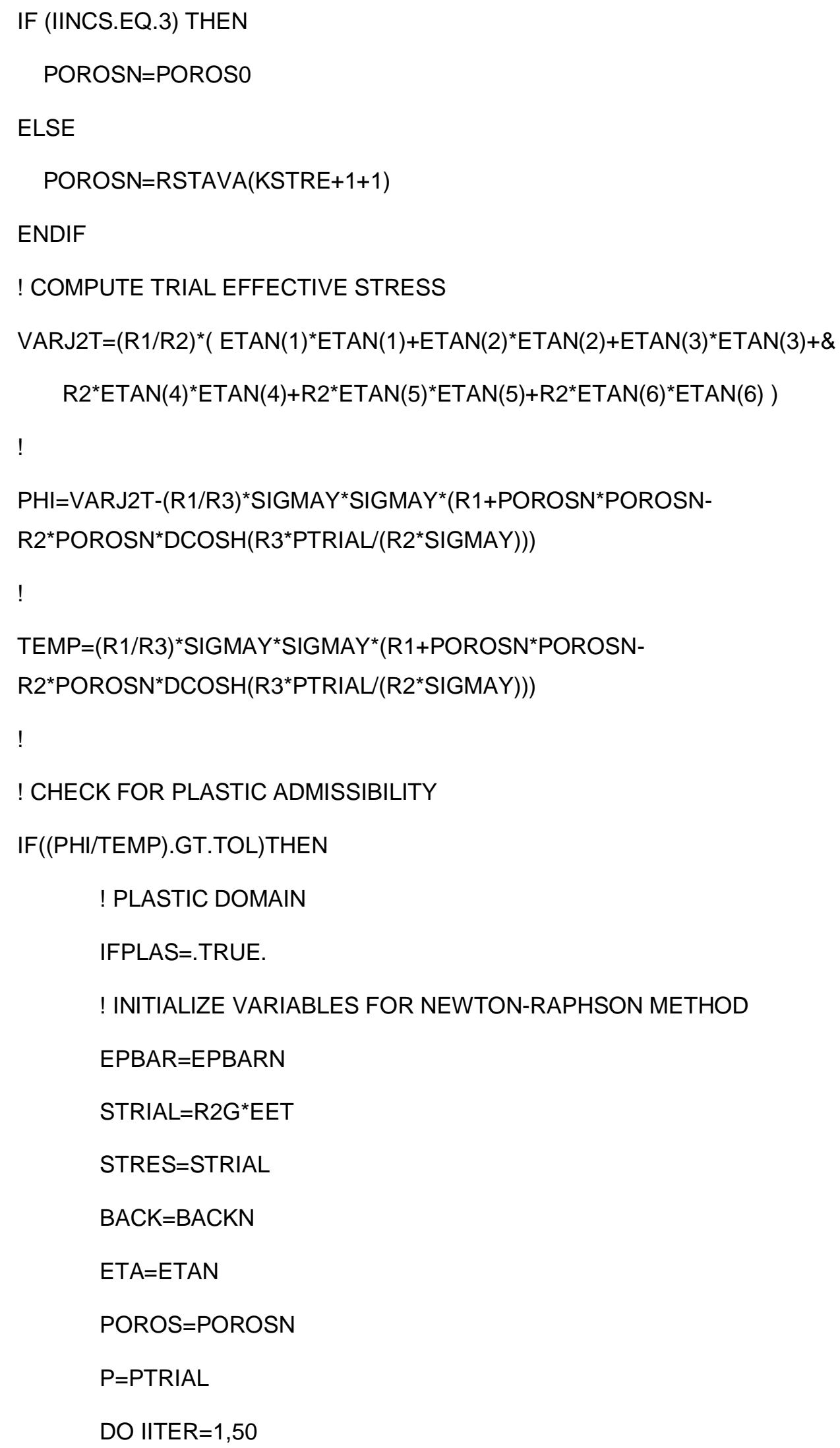




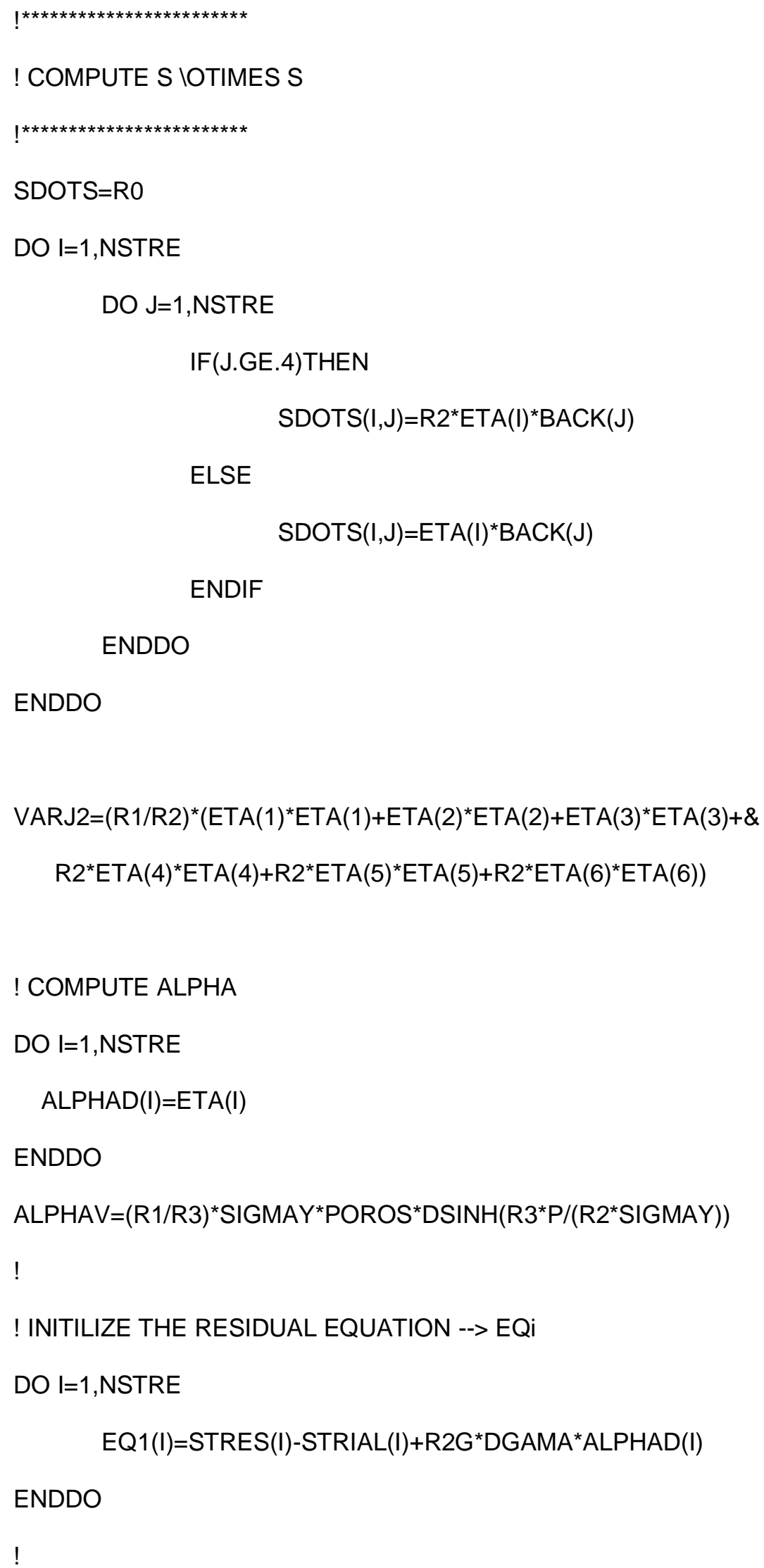




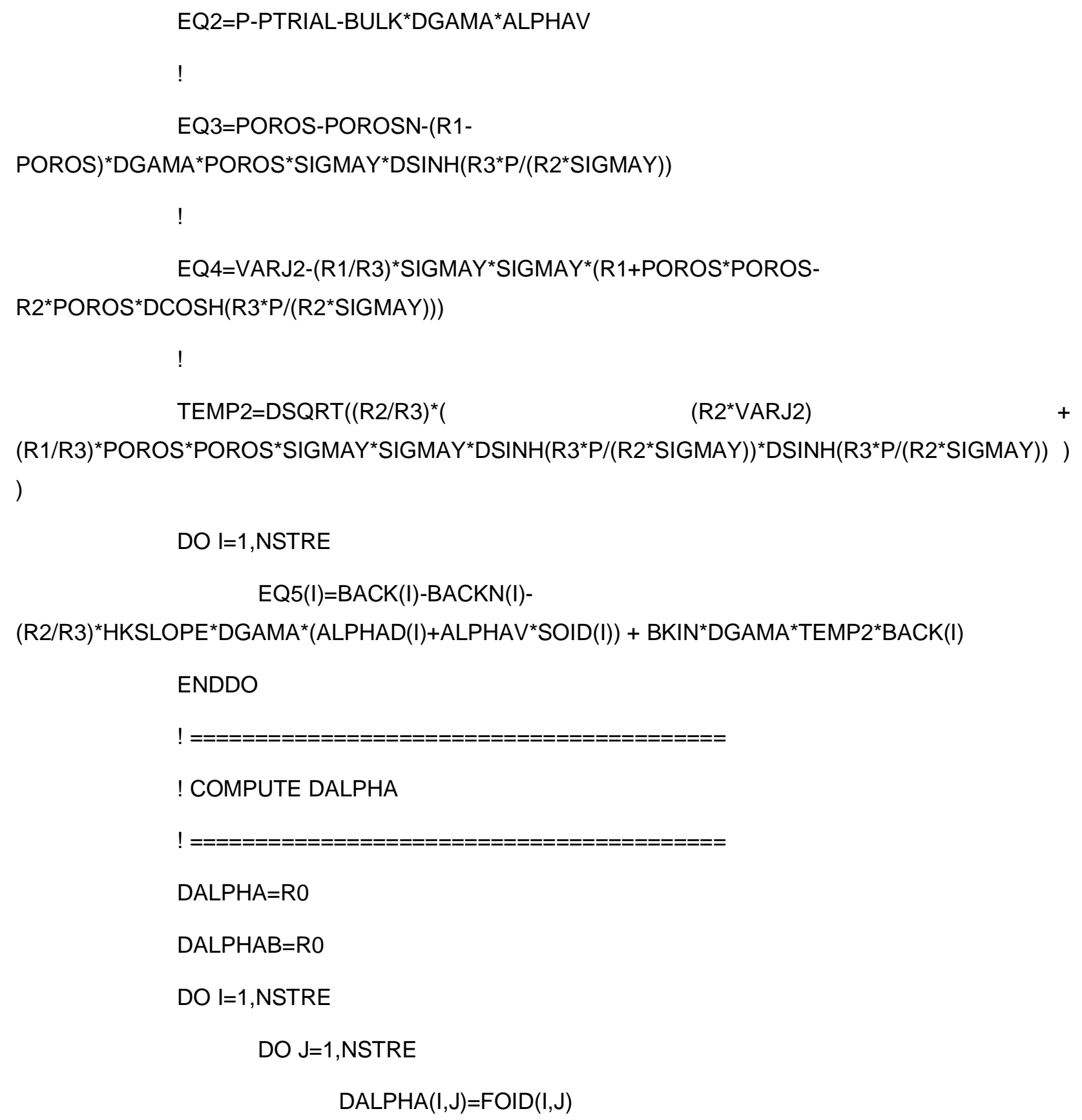




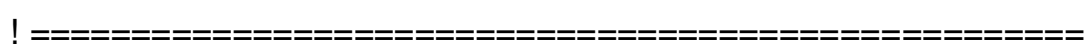

! DERIVADAS ASSOCIADAS À PRIMEIRA EQUAÇÃO DE RESÍDUO

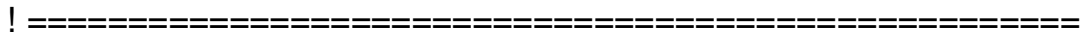

MATRIX=R0

DO l=1,NSTRE

DO J=1,NSTRE

$\operatorname{MATRIX}(\mathrm{I}, \mathrm{J})=\mathrm{FOID}(\mathrm{I}, \mathrm{J})+\mathrm{R} 2 \mathrm{G}{ }^{*} \mathrm{DGAMA}{ }^{*} \operatorname{DALPHA}(\mathrm{I}, \mathrm{J})$

ENDDO

ENDDO

$\operatorname{MATRIX}(1,7)=\mathrm{R} 0$

$\operatorname{MATRIX}(2,7)=\mathrm{R} 0$

$\operatorname{MATRIX}(3,7)=\mathrm{R} 0$

$\operatorname{MATRIX}(4,7)=\mathrm{R} 0$

$\operatorname{MATRIX}(5,7)=\mathrm{R} 0$

$\operatorname{MATRIX}(6,7)=\mathrm{R} 0$

$\operatorname{MATRIX}(1,8)=\operatorname{R} 0$

$\operatorname{MATRIX}(2,8)=\mathrm{R} 0$

$\operatorname{MATRIX}(3,8)=\mathrm{R} 0$

$\operatorname{MATRIX}(4,8)=\mathrm{R} 0$

$\operatorname{MATRIX}(5,8)=\mathrm{R} 0$

$\operatorname{MATRIX}(6,8)=\mathrm{R} 0$

$\operatorname{MATRIX}(1,9)=\operatorname{R} 2 \mathrm{G} * \operatorname{ALPHAD}(1)$

$\operatorname{MATRIX}(2,9)=\operatorname{R} 2 \mathrm{G}^{*} \operatorname{ALPHAD}(2)$

$\operatorname{MATRIX}(3,9)=R 2 \mathrm{G}^{*} \operatorname{ALPHAD}(3)$ 


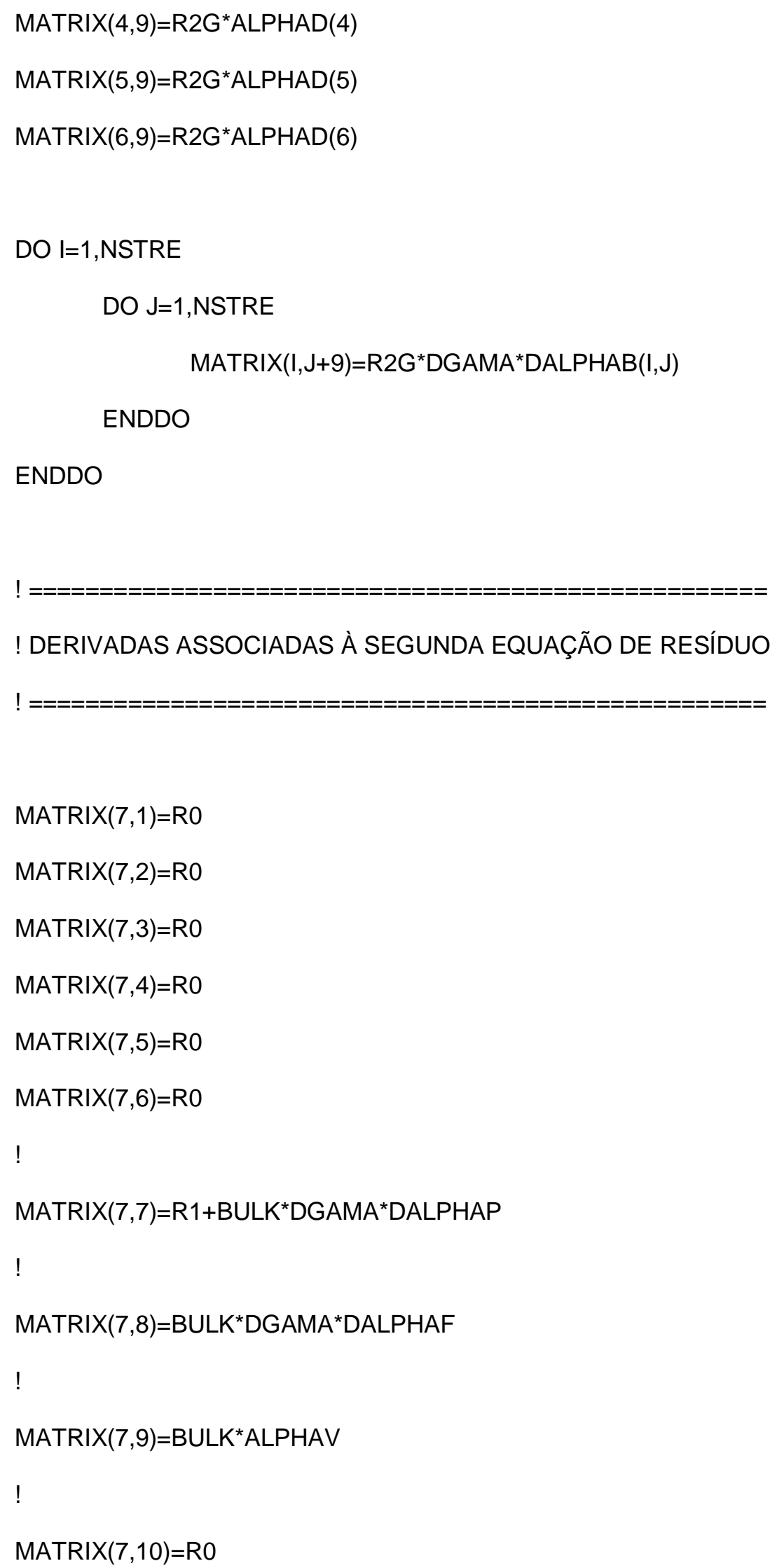




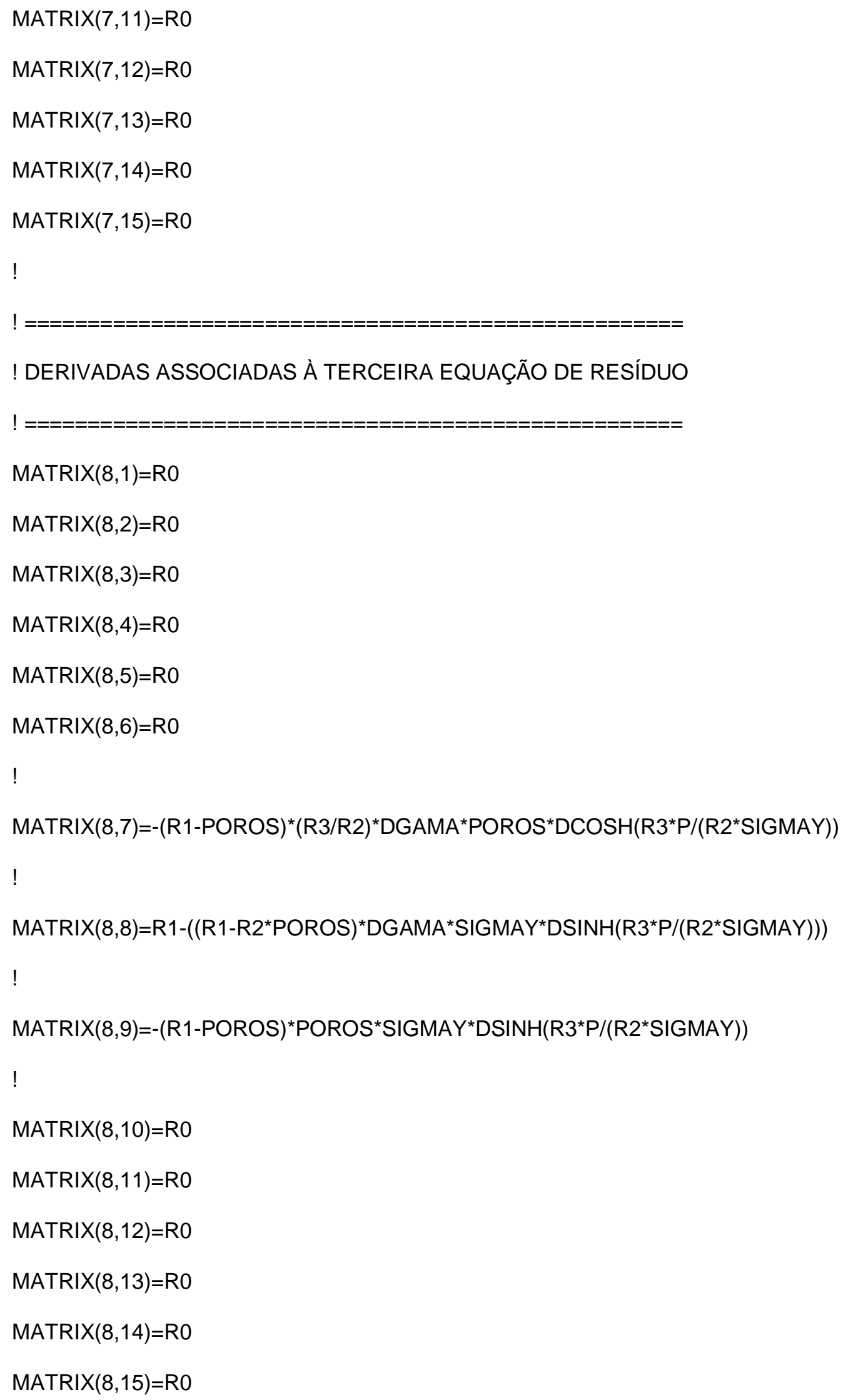




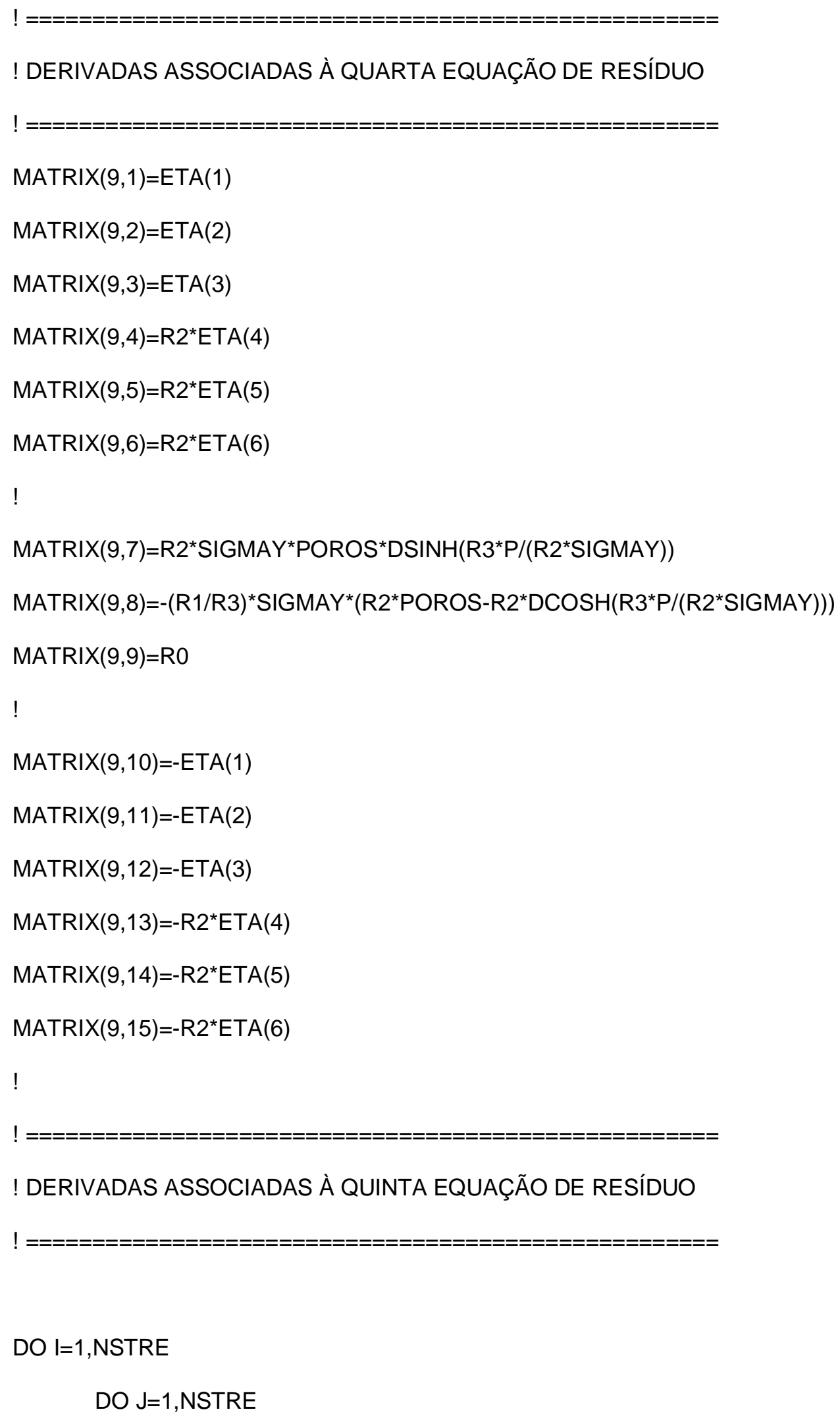




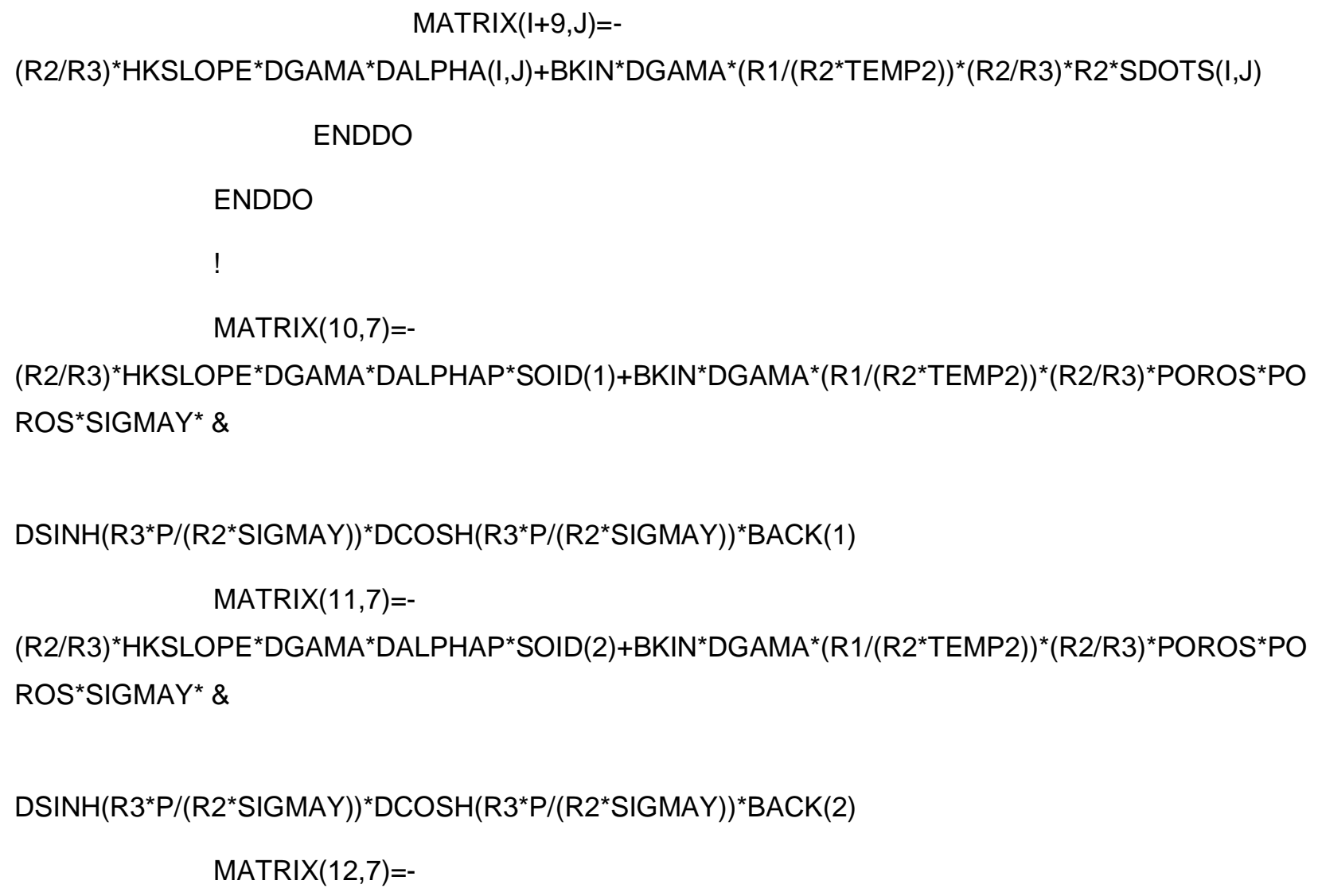


$\mathrm{DSINH}\left(\mathrm{R}{ }^{*} \mathrm{P} /\left(\mathrm{R} 2^{*} \mathrm{SIGMAY}\right)\right)^{*} \mathrm{DCOSH}\left(\mathrm{R} 3^{*} \mathrm{P} /\left(\mathrm{R} 2^{*} \mathrm{SIGMAY}\right)\right)^{*} \mathrm{BACK}(6)$

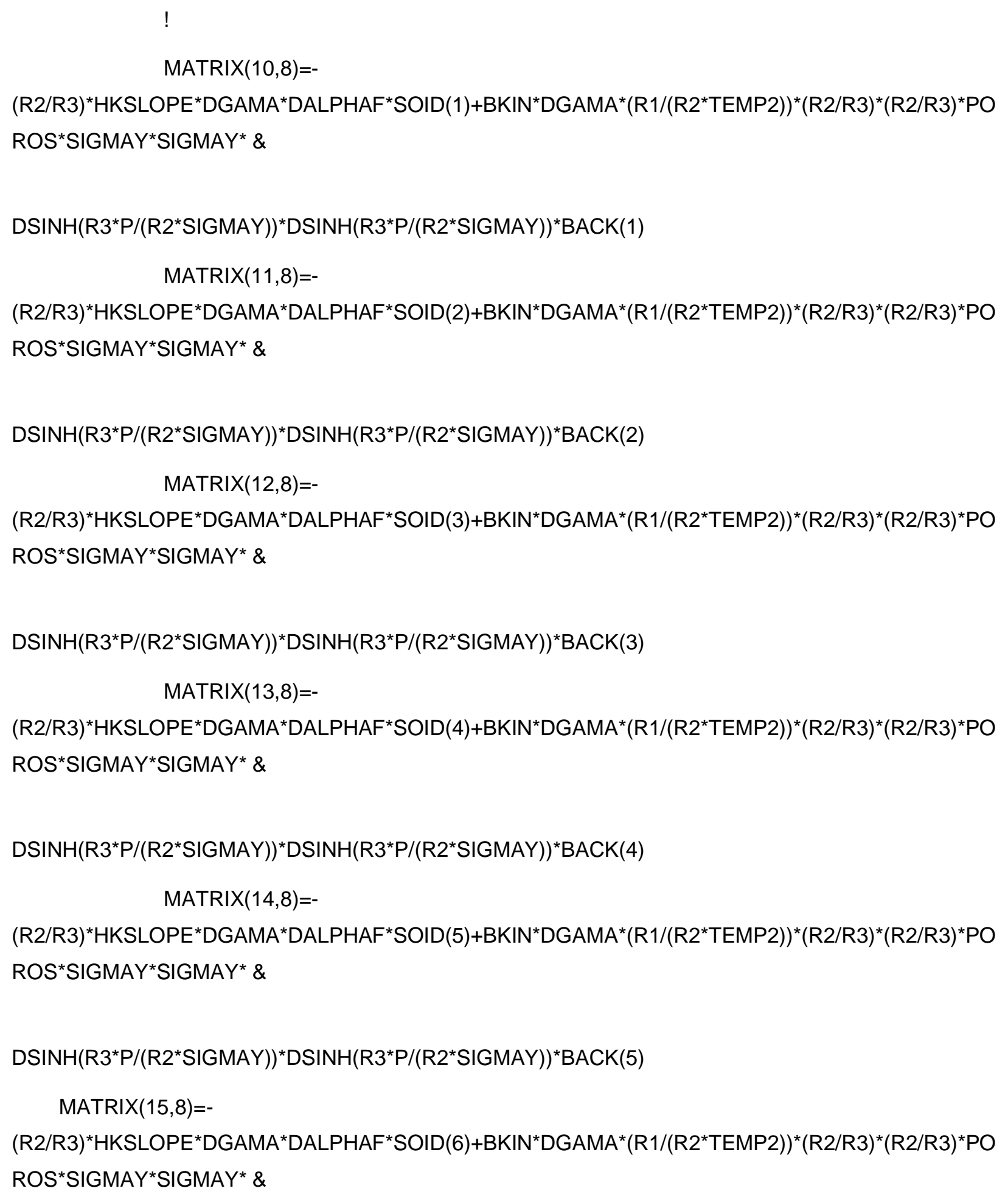


$\operatorname{DSINH}\left(\mathrm{R3}^{*} \mathrm{P} /\left(\mathrm{R} 2^{*} \mathrm{SIGMAY}\right)\right)^{*} \mathrm{DSINH}\left(\mathrm{R}{ }^{*} \mathrm{P} /\left(\mathrm{R} 2^{*} \mathrm{SIGMAY}\right)\right)^{*} \mathrm{BACK}(6)$

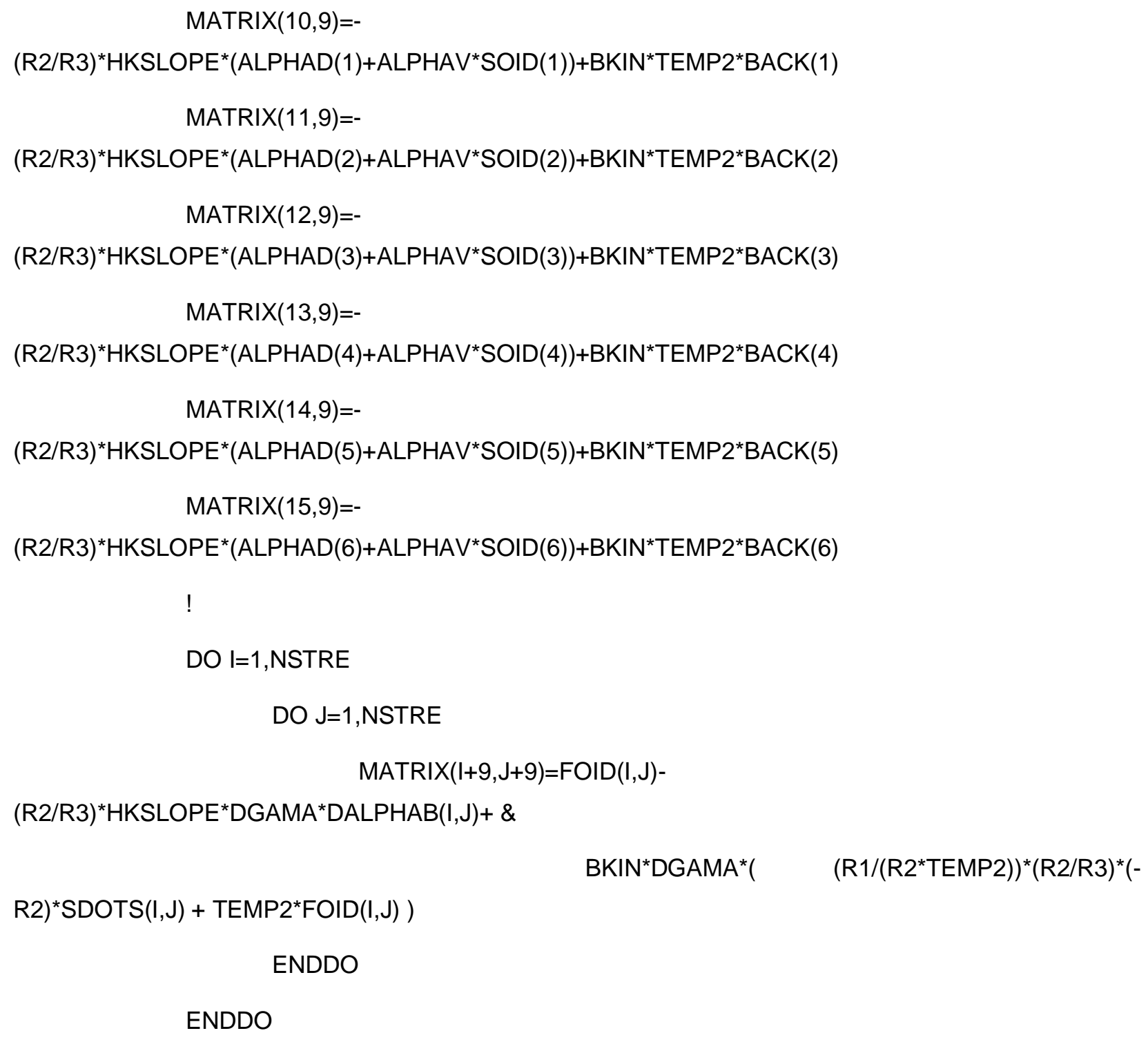




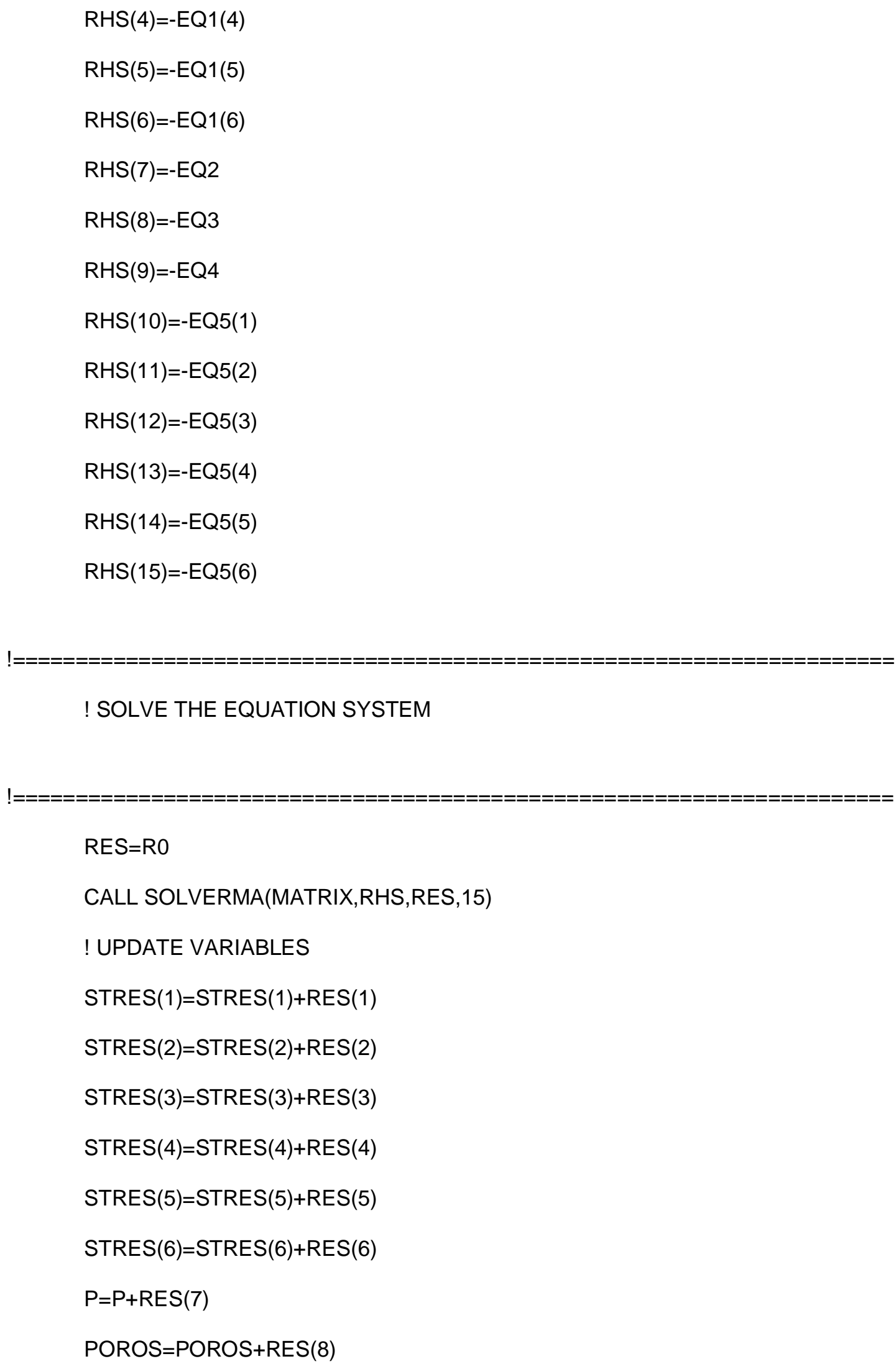


$\mathrm{DGAMA}=\mathrm{DGAMA}+\mathrm{RES}(9)$

$\operatorname{BACK}(1)=\operatorname{BACK}(1)+\operatorname{RES}(10)$

$\operatorname{BACK}(2)=\operatorname{BACK}(2)+\operatorname{RES}(11)$

$\operatorname{BACK}(3)=\operatorname{BACK}(3)+\operatorname{RES}(12)$

$\operatorname{BACK}(4)=\operatorname{BACK}(4)+\operatorname{RES}(13)$

$\operatorname{BACK}(5)=\operatorname{BACK}(5)+\operatorname{RES}(14)$

$\operatorname{BACK}(6)=\operatorname{BACK}(6)+\operatorname{RES}(15)$

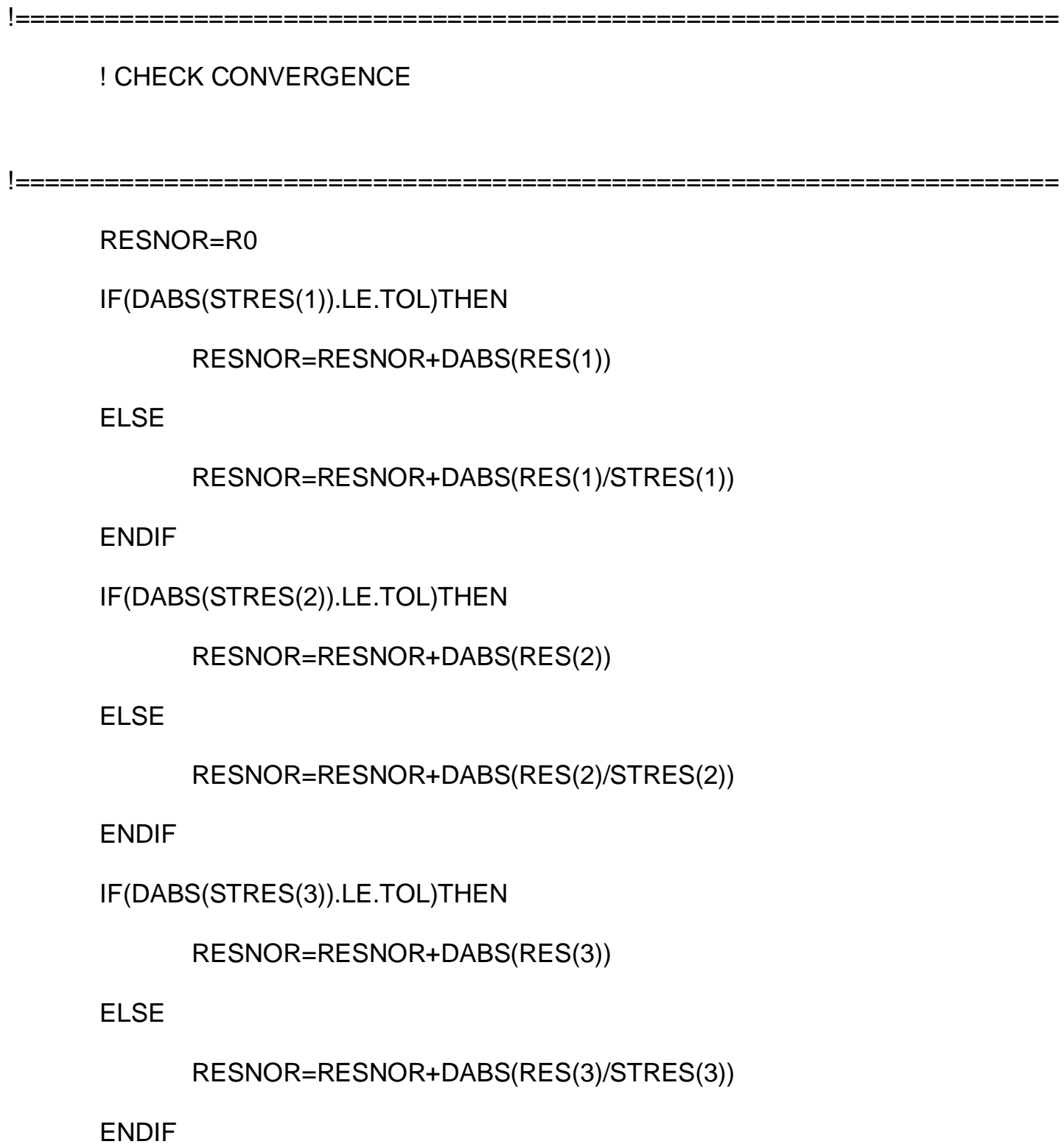




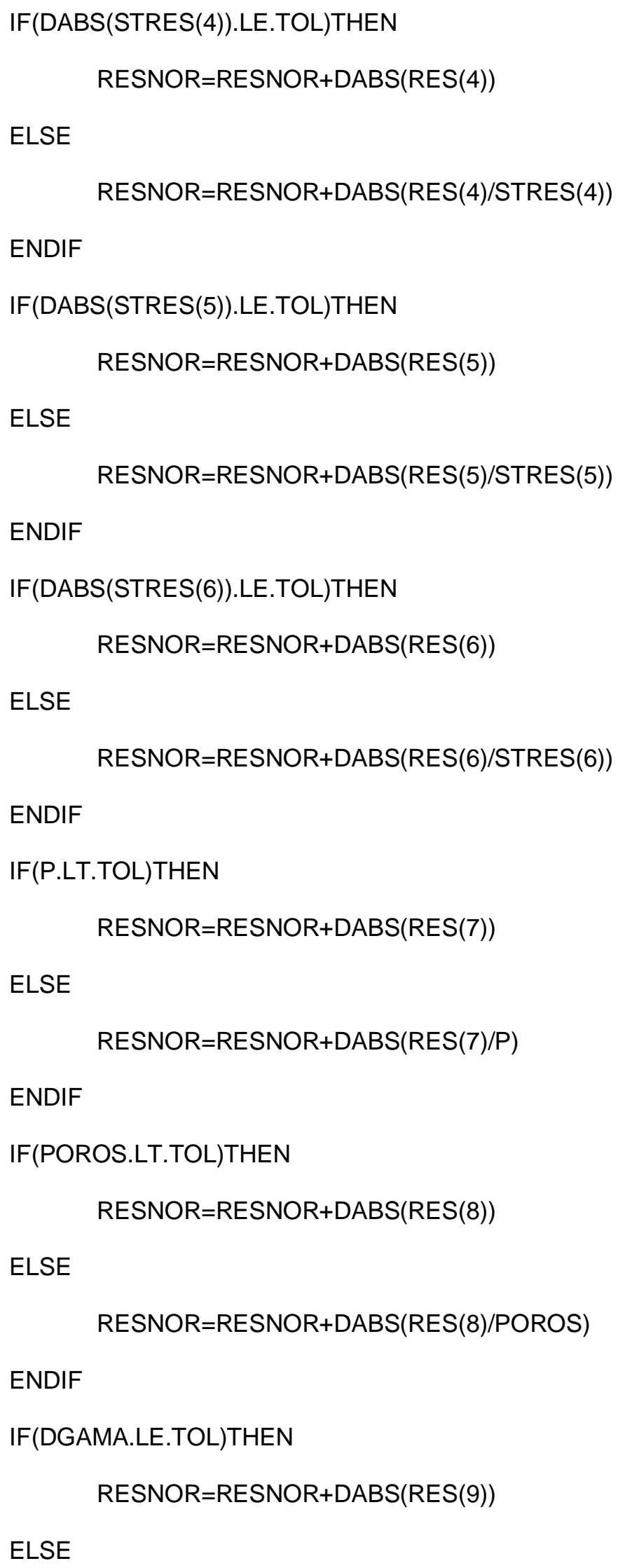




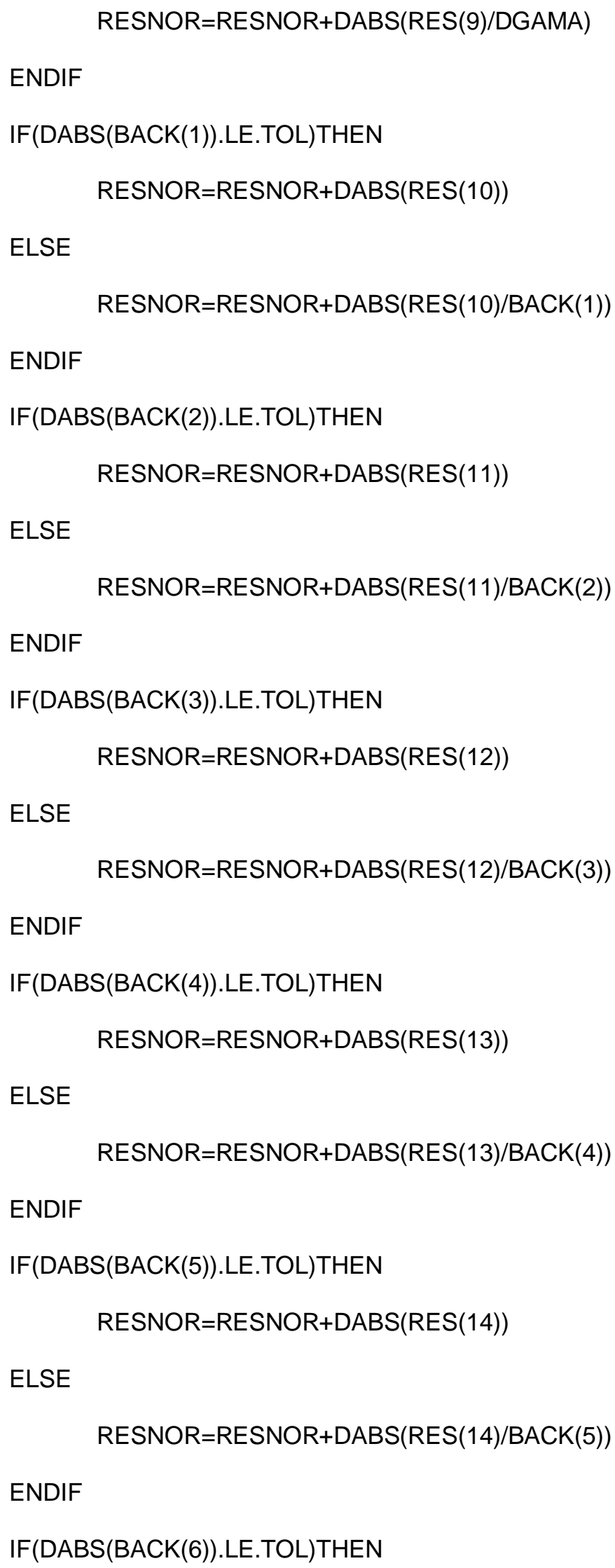




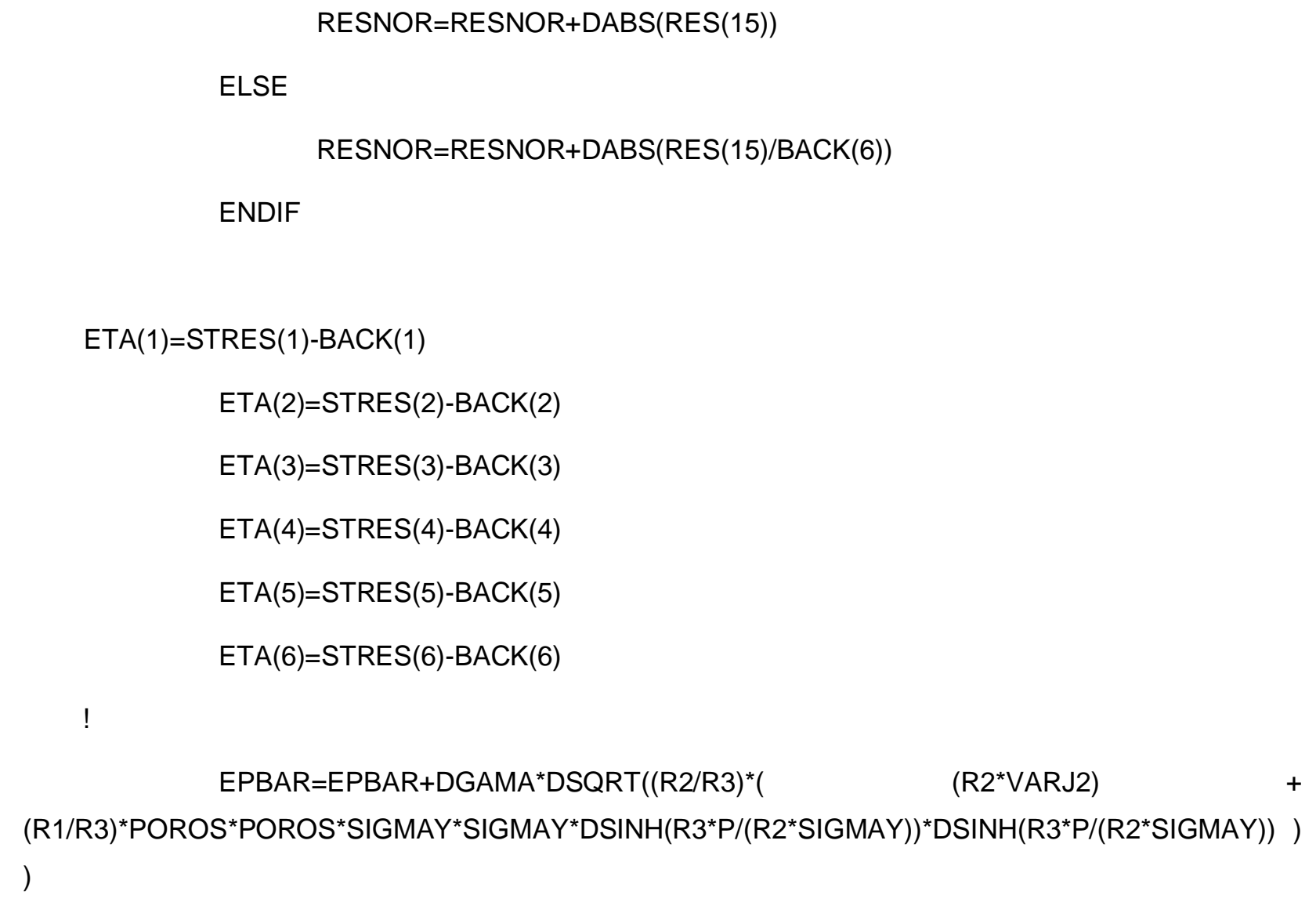

IF(RESNOR.LE.TOL)THEN

RSTAVA(1) $=(\operatorname{STRES}(1) / \mathrm{R} 2 \mathrm{G})+(\mathrm{R} 1 / \mathrm{R} 3)^{*} \mathrm{P} / \mathrm{BULK}$ $\operatorname{RSTAVA}(2)=(\mathrm{STRES}(2) / \mathrm{R} 2 \mathrm{G})+(\mathrm{R} 1 / \mathrm{R} 3)^{*} \mathrm{P} / \mathrm{BULK}$ $\operatorname{RSTAVA}(3)=(\operatorname{STRES}(3) / \mathrm{R} 2 \mathrm{G})+(\mathrm{R} 1 / \mathrm{R} 3)^{*} \mathrm{P} / \mathrm{BULK}$ $\operatorname{RSTAVA}(4)=(\operatorname{STRES}(4) / \mathrm{R} 2 \mathrm{G}){ }^{*} \mathrm{R} 2$ !!!!!!!!!!!!!!!!!!!!!!!!!!!!!!!!!!!!!!!! ATENÇÃO!!!!!!!!!!!!!!!!! ATENÇÃO!!!!!!!!!!!!!!!!!

$\operatorname{RSTAVA}(6)=(\operatorname{STRES}(6) / \mathrm{R} 2 \mathrm{G}){ }^{\star} \mathrm{R} 2$ ATENÇÃO!!!!!!!!!!!!!!!!! 


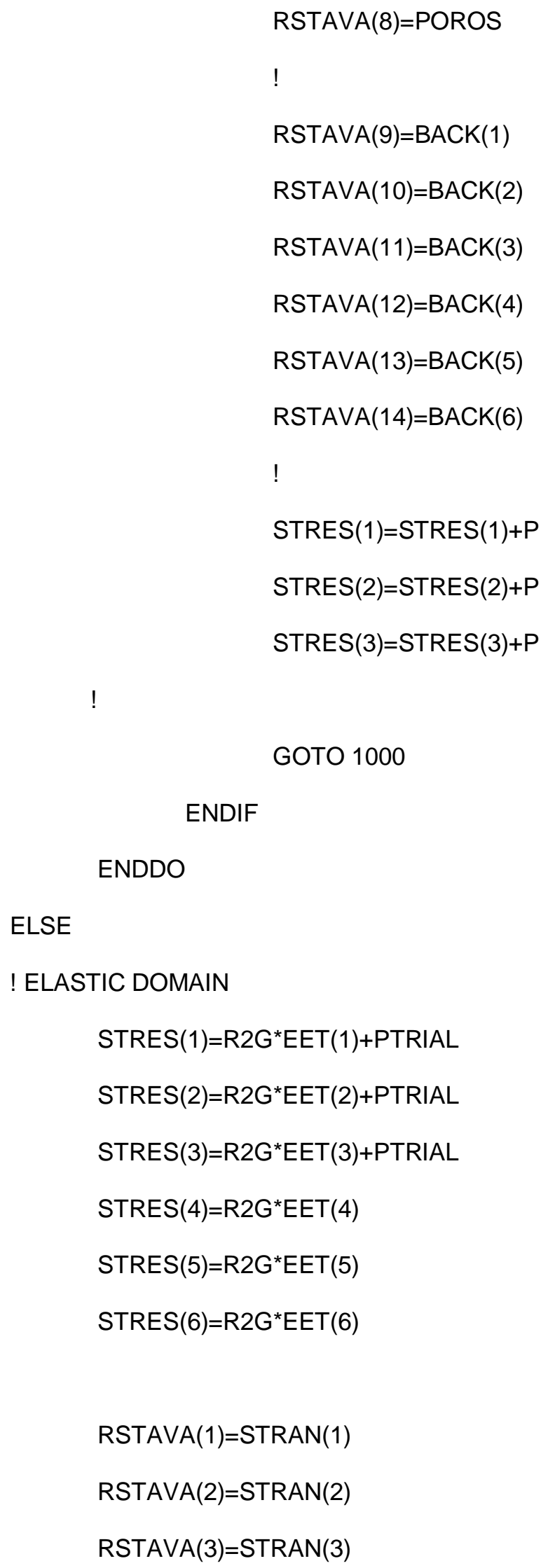




$\operatorname{RSTAVA}(4)=\operatorname{STRAN}(4)$
$\operatorname{RSTAVA}(5)=\operatorname{STRAN}(5)$
$\operatorname{RSTAVA}(6)=\operatorname{STRAN}(6)$
RSTAVA$(7)=\mathrm{EPBARN}$
RSTAVA(8)=POROSN
RSTAVA(9)=BACKN(1)
RSTAVA(10)=BACKN(2)
RSTAVA(11)=BACKN(3)
RSTAVA(12)=BACKN(4)
RSTAVA(13)=BACKN(5)
RSTAVA(14)=BACKN(6)
ENDIF
1000 CONTINUE
LALGVA(1)=IFPLAS
LALGVA(2)=SUFAIL
RETURN
END

